Universität Potsdam - Institut für Mathematik

Arbeitsgruppe

"Partielle Differentialgleichungen und Komplexe Analysis"

\title{
Operator Algebras With Symbol Hierarchies On Manifolds With Singularities
}

\author{
Bert-Wolfgang Schulze
}

Preprint 99/30

December 1999

$\begin{array}{ll}\text { Herausgeber: } & \begin{array}{l}\text { Arbeitsgruppe } \\ \text { "Partielle Differentialgleichungen und Komplexe Analysis" } \\ \\ \text { am Institut für Mathematik der Universität Potsdam }\end{array} \\ & \begin{array}{l}\text { Postfach } 601553 \\ \text { Adresse: }\end{array} \\ & +4415 \text { Potsdam } \\ \text { Telefon: } & +49-331-9771269 \\ \text { Fax: } & \text { wallroth@math.uni-potsdam.de } \\ \text { Email: } & \end{array}$

ISSN $1437-739 X$ 


\title{
OPERATOR ALGEBRAS WITH SYMBOL HIERARCHIES ON MANIFOLDS WITH SINGULARITIES
}

\author{
B.-W. SCHULZE
}

\begin{abstract}
Problems for elliptic partial differential equations on manifolds $M$ with singularities $M^{\prime}$ (here with piece-wise smooth geometry) are studied in terms of pseudo-differential algebras with hierarchies of symbols that consist of scalar and operator-valued components. Classical boundary value problems (with or without the transmission property) belong to the examples. They are a model for operator algebras on manifolds $M$ with higher "polyhedral" singularities. The operators are block matrices that have upper left corners containing the pseudo-differential operators on the regular part $M \backslash M^{\prime}$ (plus certain Mellin and Green summands) and are degenerate (in stretched coordinates) in a typical way near $M^{\prime}$. By definition $M^{\prime}$ is again a manifold with singularities. The same is true of $M^{\prime \prime}$, and so on. The block matrices consist of trace, potential and Mellin and Green operators, acting between weighted Sobolev spaces on $M^{(j)}$ and $M^{(k)}$, with $0 \leq j, k \leq$ ord $M$; here $M^{(0)}$ denotes $M, M^{(1)}$ denotes $M^{\prime}$, etc. We generate these algebras, including their symbol hierarchies, by iterating so-called "edgifications" and "conifications" of algebras that have already been constructed, and we study ellipticity, parametrices, and Fredholm property within these algebras.
\end{abstract}

\section{INTRODUCTION}

The study of partial differential equations on non-smooth configurations or with discontinuous data has a long history, cf. Hertz [21], Carleman [6], Zaremba [85], Wiener [82], Lamé [28], Sommerfeld [75], Keller [24], Sobolev [74], Kondrat'ev [25], Teleman [77], or the author's joint book with Wildenhain [72]. It was discovered very early that singularities of the geometry (e.g., of corner or cusp type) drastically affect the behaviour of solutions.

The singular configurations, here understood in a rather specific sense and briefly referred to as manifolds with singularities, may be characterised by singular geometries, for instance, of polyhedral type, or by non-compact "exits" to infinity. Also other more complicated structures are subsumed under this notation, e.g., "hedgehog-shaped" spaces or cracks, defined as complements of embedded (singular) hypersurfaces in a $C^{\infty}$ manifold.

Motivated by relevant applications in physics, engineering, applied sciences and mathematics (analysis, mathematical physics, geometry and topology) there is a vast stream of investigations on different aspects of analysis near singularities (see, for instance, the bibliographies in [26], [58], [31], [37]).

Date: May 8, 2000.

1991 Mathematics Subject Classification. 35S15, 58G20,46E35, 46H35.

Key words and phrases. pseudo-differential operators, stratified spaces, manifolds with edge and corner singularities, operator algebras, elliptic operators, boundary value problems.

Acknowledgement. The author thanks M. Korey, T. Krainer, J. Seiler and N. Tarkhanov, all of the University of Potsdam, for valuable remarks on the manuscript. 
Many of these papers are devoted to singular problems in a specific context, others to more general structure theories for large classes of operators, under the aspect of operator algebras. The second category is particularly adapted to elliptic (and also parabolic) equations. Some ideas from that field have found numerous applications and have, for instance, played a role in the development of the pseudodifferential calculus, in boundary value problems, or in the Atiyah-Singer index theorem.

Analysis near singularities is much more complicated than that over the smooth parts of a configuration, and the variety of new structures and phenomena that arise is enormous.

For instance, the task to extend the basic elements of the microlocal analysis on smooth manifolds to a configuration with even simplest geometric singularities requires new specific calculi. Not very much is really known about hyperbolic equations on polyhedral spatial regions. Also the generalisation of the classical index theory of elliptic operators from the closed compact $C^{\infty}$ case to (non-compact or) singular manifolds is still far from satisfactory completion.

In the past few years the development in these directions has accelerated considerably, and many exciting partial results have been obtained. There are, in fact, several strategies to extend the standard concept of ellipticity and index of Fredholm operators from the smooth, compact case to the singular case.

In the present paper we discuss a program for manifolds with polyhedral singularities as well as certain other classes of non-compact manifolds. By polyhedral we mean singularities of conical, edge, corner, etc. type with a certain transversality of intersections of faces near the singularities. Also the case of manifolds with boundary is included as a subclass of manifolds with edges. The "non-transversal" case corresponds to cuspidal singularities; a modification of the program also applies to this situation, cf. Schulze and Tarkhanov [70].

The various possible operator calculi on a configuration with singularities are determined by corresponding symbols on associated stretched spaces that are degenerate near the singularities in a specific way. For instance, if we consider $\left(\overline{\mathbb{R}}_{+}\right)^{k} \times \Sigma \times \Omega$ for some $k \in \mathbb{N}$ and open sets $\Sigma \subseteq \mathbb{R}^{n}, \Omega \subseteq \mathbb{R}^{q}$, with the variables $(r, x, y)$ and covariables $(\varrho, \xi, \eta)$, the symbols may have the form

$$
p(r, x, y, \varrho, \xi, \eta)=w(r) \tilde{p}(r, x, y, \varphi(r) \varrho, \xi, \psi(r) \eta) .
$$

Here, the weight factor $w(r)$ is in $C^{\infty}\left(\left(\mathbb{R}_{+}\right)^{k}\right)$ and the (say classical) symbols $\tilde{p}(r, x, y, \tilde{\varrho}, \xi, \tilde{\eta})$ are smooth up to $r=0$, where $\varphi(r) \in C^{\infty}\left(\left(\mathbb{R}_{+}\right)^{k}, \mathbb{R}^{k}\right)$ and $\psi(r) \in C^{\infty}\left(\left(\mathbb{R}_{+}\right)^{k}, \mathbb{R}^{q}\right)$ are given functions and $\varphi \varrho=\sum_{j=1}^{k} \varphi_{j} \varrho_{j}, \psi \eta=\sum_{l=1}^{q} \psi_{l} \eta_{l}$. In typical cases we have $w(r)=\left(r_{1} r_{2} \ldots r_{k}\right)^{-\mu}$ for $\mu=\operatorname{ord} p, \varphi_{1}(r)=r_{1}$, $\varphi_{2}(r)=r_{1} r_{2}, \ldots, \varphi_{k}(r)=r_{1} r_{2} \ldots r_{k}$, and similar natural monomials $\psi_{l}(r)$ in $r, l=1, \ldots, q$. In more complicated examples the functions $w, \varphi, \psi$ also depend on the other variables, and $\tilde{p}$ may be $C^{\infty}$ only in $r \in\left(\mathbb{R}_{+}\right)^{k}$, with a controlled behaviour near 0 .

Symbols reflect specific geometric information, and this differentiates the behaviour of conical singularities and that of various types of cuspidal singularities. Furthermore, conical and edge singularities give rise to different types of degenerate symbols.

A productive source of notational confusion is the fact that often the singular manifolds are tacitly identified with their stretched spaces; then the calculus for conical singularities appears as an aspect of boundary value problems. The "usual" 
boundary value problems (like the Dirichlet problem for Laplace operators) are of a rather different behaviour; they fit into the concept of edge singularities. Boundary value problems, of course, contain boundary conditions and edge problems edge conditions. (This is also the case for a global scenario with analogues of APS conditions, cf. the remarks below and the papers [68], [47], [64].) For polyhedral singularities there are edge conditions on all faces of the lower-dimensional skeleta. (Clearly, everything depends on the corresponding choice of degenerate operators; here we assume symbols of the form (0.1) with the above monomials $\varphi$ and $\psi$.) The various trace, potential and Green operators also contribute to the nature of the upper left corners in the corresponding block matrices; of special importance is that they generate ideals of smoothing but non-compact operators and contribute to the index theory, even if one disregards the entries with the additional conditions.

We mainly stress in this paper the "analytic" part of the theory, since we intend to motivate the approach in analogy to Gelfand's program [14], which was originally formulated for elliptic boundary value problems. This can partly be summarised as follows. Given a space $X$ (in this case a compact $C^{\infty}$ manifold with $C^{\infty}$ boundary), we consider the class of all elliptic boundary value problems on $X$, with ellipticity of the boundary data as the Shapiro-Lopatinskij condition. We then have

(i) an operator algebra $\mathcal{L}(X)$ generated by the differential boundary value problems,

(ii) a principal symbol map $\sigma: \mathcal{L}(X) \rightarrow \operatorname{symb} \mathcal{L}(X)$ which determines ellipticity,

(iii) a scale of Sobolev spaces $\mathcal{H}^{s}, s \in \mathbb{R}$, such that the operators $A \in \mathcal{L}(X)$ induce continuous maps $A: \mathcal{H}^{s} \rightarrow \mathcal{H}^{s-\mu}$ for $\mu=\operatorname{ord} A$,

(iv) equivalence of ellipticity and the Fredholm property of $A$.

The standard theory of elliptic boundary value problems shows that the index ind $A=\operatorname{dim} \operatorname{ker} A-\operatorname{dim}$ coker $A$ only depends on $\sigma(A)$, more generally on the stable homotopy class $[\sigma(A)]$ of $\sigma(A)$ through elliptic symbols. The idea was to express ind $A$ purely in terms of $[\sigma(A)]$. For the simpler situation of closed compact $X$ the result is just the index formula of Atiyah and Singer [3]. For the case of elliptic differential boundary value problems (with ellipticity in the Shapiro-Lopatinskij sense) the index formula was extended by Atiyah and Bott [1], whereas for pseudodifferential boundary value problems with the transmission property by Boutet de Monvel [5]. The general case (without the transmission property) was treated in the author's joint paper with Rempel [45]. Boundary value problems for elliptic operators that do not possess Shapiro-Lopatinskij elliptic boundary conditions (like Dirac operators or other geometric operators) belong to a branch of the index theory with emphasis on other features than symbol structures, cf. Atiyah, Patodi and Singer [2]. However, as it finally turns out, such problems may also be understood in the context of a corresponding symbol philosophy from an enlarged concept of ellipticity in a suitable operator algebra, cf. [68], [47], or [64].

The program to study operator algebras on a singular manifold $X$ is particularly instructive in the comparatively simple case of boundary value problems. Then $X$ is a compact $C^{\infty}$ manifold with $C^{\infty}$ boundary $Y$; differential operators on $X$ are assumed to have smooth coefficients up to $Y$. A convenient pseudo-differential algebra of boundary value problems is due to Boutet de Monvel [5]; see also the author's joint book with Rempel [44]. A basic assumption on the symbols involved in this algebra of pseudo-differential operators on int $X$ is the transmission property with 
respect to the boundary. Let $L_{c l}^{\mu}(\text { int } X)_{\text {trans }}$ be the space of all classical pseudodifferential operators of order $\mu$ on int $X$ with local symbols having the transmission property with respect to $Y$, and compare $L_{\mathrm{cl}}^{\mu}(\operatorname{int} X)_{\text {trans }}$ with $L_{\mathrm{cl}}^{\mu}(\operatorname{int} X)_{\mathrm{smooth}}$, the space of all classical pseudo-differential operators of order $\mu$ on int $X$ with local symbols being smooth up to $Y$. The space $L_{c l}^{\mu}(\text { int } X)_{\text {smooth }}$ is much larger than $L_{\mathrm{cl}}^{\mu}(\text { int } X)_{\text {trans }}$, though rational functions in the covariables $\xi$ (and as such, those algebraically generated by polynomials) have the transmission property. On the one hand this shows that the transmission property is reached when we "generate" pseudo-differential operators on $X$ by vector fields that are smooth up to the boundary; on the other hand, most classical symbols fail to have the transmission property (for example, symbols which equal $|\xi|^{\mu}$ for large $|\xi|$, for $\mu \notin 2 \mathbb{Z}$ ). General symbols are important in many applications, e.g., to understand mixed elliptic problems (like the Zaremba problem) in a pseudo-differential context. It is shown in [60] that they can be interpreted in connection with edge-degenerate symbols when $X$ is regarded as a manifold with edge $Y$. However, even if we suppose the transmission property, we at once face another problem when the operators are not differential, namely to establish an operator convention that produces continuous operators in Sobolev spaces $H^{s}(X)$. The usual rule in boundary value problems is to form $\mathrm{r}^{+} \mathrm{Pe}^{+}$ with $\mathrm{e}^{+}$being the operator of extension by zero from int $X$ to the double $2 X, \mathrm{r}^{+}$the operator of restriction to int $X$, and $P$ an extension of the given pseudo-differential operator to $2 X$. This looks rather brutal and can hardly be recognised as an intrinsic operator convention. For operators without the transmission property such a rule becomes even more obscure and then also the Sobolev spaces $H^{s}(X)$ lose their predominance for the calculus. Nevertheless the pseudo-differential calculus of boundary value problems is an excellent guideline for establishing wedge theories, while the boundary symbol calculus is a model for cone theories. The calculus without the transmission property of the author [60] that employs a refinement of an algebra of Eskin [9] on the half-axis can be viewed as a model for wedge theories in general, though further essential inventions are necessary for non-trivial wedges, cf. [56], [64]. An iteration of cone and wedge theories of first order is constructed in Schulze [59]. This gives rise to a second order corner pseudo-differential calculus with a three-component symbol hierarchy, cf. [59]. The case of boundary value problems for corner manifolds (where the boundary has corners of second order) with the transmission property with respect to the smooth parts of the boundary is treated in Schulze [66]. The present paper contains a construction for the general case.

In Section 1 we fix the type of singularities by local charts and specific transition maps. Section 2 formulates "edgification": a wedge pseudo-differential calculus based on an already established cone calculus. In Section 3 we give the cone calculus in terms of the algebras on manifolds of lower singularity orders, the "conification". The iteration starts from the cone algebra for a smooth base manifold, cf. [58], [63]. Essential technical tools on pseudo-differential operators with operator-valued symbols as they are used here may be found in [7], [73] and [27].

Let us finally note that, in spite of the complexity of the resulting operator algebras, the nature of symbols remains simple and transparent. Each edgification yields a pair of new symbols that is to be added to the symbols from the steps before, namely a Fuchs symbol and an edge symbol; the latter one governs (in the elliptic theory) the extra data along the edge. Each conification contributes again a pair, a Fuchs symbol and a conormal symbol that encodes (in the elliptic 
theory) global spectral information along the (singular) cone base and contains an additional weight.

\section{The PRogram of CONIFICATIONS AND EDGiFications}

To fix our notation we first prepare some material on singular (stratified) spaces.

Spaces $M$ with polyhedral singularities that we have in mind here will be simply called manifolds with singularities (though they are pseudo-manifolds in general). Such spaces $M$ (throughout the exposition assumed to be locally compact and paracompact) are $C^{\infty}$ outside a subset $M^{\prime}$ of singular points and locally near $M^{\prime}$ described by cones $X^{\Delta}=\left(\overline{\mathbb{R}}_{+} \times X\right) /(\{0\} \times X)$ or wedges $Z^{\Delta} \times \Omega$ for open $\Omega \subseteq \mathbb{R}^{q}$, $q \geq 1$. The bases $X, Z$ of the involved cones are assumed to be compact manifolds with singularities of lower order, cf. the definition below. Thus, starting from $\overline{\mathbb{R}}_{+}$ and arbitrary closed, compact $C^{\infty}$ manifolds $X, Z$ and open sets $\Omega \subseteq \mathbb{R}^{q}, q \in \mathbb{N}$, we get inductively every such $M$. Examples are manifolds with conical singularities, edges, corners, or smooth manifolds with boundary.

The compactness of the bases of the cones is essential for the analysis below (though it is not really important to define singular charts on $M$; concerning general material on stratified spaces, $\mathrm{cf}$. the book of Goresky and MacPherson [20]).

A point $x_{0} \in M$ with a neighbourhood $V \sim X^{\Delta}\left(W \sim Z^{\Delta} \times \Omega\right)$ is called a corner point (edge point) and $V$ a corner neighbourhood ( $W$ a wedge neighbourhood) of $x_{0}$. Of course, we can unify the notation by considering points $x_{0} \in M^{\prime}$ with neighbourhoods $U \subseteq M$ that are homeomorphic to $X_{1}^{\Delta} \times \Omega_{1}$ for a certain $X_{1}$ with singularities and open $\Omega_{1} \subseteq \mathbb{R}^{q_{1}}$ for any $q_{1} \geq 0$. Both $X_{1}$ and $q_{1}$ may depend on $x_{0}$. The corresponding maps $\chi: U \rightarrow X_{1}^{\Delta} \times \Omega_{1}$ are called singular charts (standard charts on $M \backslash M^{\prime}$ are called regular). Let us pass to the stretched space $\overline{\mathbb{R}}_{+} \times X_{1} \times \Omega_{1}$.

Each $x_{1} \in X_{1}^{\prime}$ has a neighbourhood that is homeomorphic to $X_{2}^{\Delta} \times \Omega_{2}$ for suitable $X_{2}, \Omega_{2}$. This yields a stretched space $\overline{\mathbb{R}}_{+} \times X_{2} \times \Omega_{2}$. For each fixed $x_{2} \in X_{2}^{\prime}$ we can do the same to get $X_{3}, \Omega_{3}$ and $\overline{\mathbb{R}}_{+} \times X_{3} \times \Omega_{3}$. This gives us a successive procedure to generate $X_{j}, \Omega_{j}$ and $\overline{\mathbb{R}}_{+} \times X_{j} \times \Omega_{j}$ for every choice of points $x_{j-1} \in X_{j-1}^{\prime}$ (where $X_{0}^{\prime}$ is identified with $M^{\prime}$ ). There is then a minimal number $k \in \mathbb{N}$ such that $X_{k}^{\prime}$ is empty. For each sequence $\left(x_{j}\right)_{0 \leq j \leq k-1}$ of this kind we can form the space $\left(\overline{\mathbb{R}}_{+}\right)^{k} \times X_{k} \times \Omega_{1} \times \ldots \times \Omega_{k}$. We assume here that the connected components of $X_{k}$ have all the same dimension; cf. also Remark 1.1 below. Then $X_{k}$ is a compact $C^{\infty}$ manifold. For $\operatorname{dim} X_{k}=0$ we get a disjoint union of a finite number of points. We define the order of singularity ord $M$ to be the maximal occurring $k$ when $\left(x_{j}\right)_{0 \leq j \leq k-1}$ varies over all such sequences.

If $M, \widetilde{M}$ are manifolds with singularities, also $M \times \widetilde{M}$ is a manifold with singularities, and we have ord $(M \times \widetilde{M})=\operatorname{ord} M+\operatorname{ord} \widetilde{M}$. In particular, if $\widetilde{M}$ is a $C^{\infty}$ manifold (for instance, closed and compact, or an open set in $\mathbb{R}^{q}$ ) we have ord $\widetilde{M}=0$, i.e., ord $(M \times \widetilde{M})=$ ord $M$. In the case $\widetilde{M}=\left(\overline{\mathbb{R}}_{+}\right)^{l}$ we have ord $\widetilde{M}=l$ and hence ord $\left(M \times\left(\overline{\mathbb{R}}_{+}\right)^{l}\right)=$ ord $M+l$.

Let us now specify the nature of the transition maps of local coordinates belonging to a system of charts $\chi: U \rightarrow D$ on $M$. It suffices to consider the singular charts. Suppose by induction that we have already defined the set of isomorphisms $\operatorname{Iso}(A, B)$ (that are, in particular, homeomorphisms) and also the spaces $C^{\infty}(\Sigma$, Iso $(A, B))$ for manifolds with singularities $A, B$ with ord $A$, ord $B<$ ord $M$ and $\Sigma \subseteq \mathbb{R}^{q}$ open. Isomorphisms are diffeomorphisms for ord $A=$ ord $B=0$. Moreover, a homeomorphism $\kappa: \overline{\mathbb{R}}_{+} \rightarrow \overline{\mathbb{R}}_{+}$belongs to Iso( $\left(\overline{\mathbb{R}}_{+}, \overline{\mathbb{R}}_{+}\right)$if it is the restriction 
of a diffeomorphism $\kappa: \mathbb{R} \rightarrow \mathbb{R}$ to $\overline{\mathbb{R}}_{+}$. It is clear what $C^{\infty}\left(\Sigma\right.$, Iso $\left.\left(\overline{\mathbb{R}}_{+}, \overline{\mathbb{R}}_{+}\right)\right)$means. If $\chi_{j}: U \rightarrow X_{j}^{\Delta} \times \Omega_{j}, j=1,2$, are singular charts, we require that the transition map $\chi_{2} \chi_{1}^{-1}$ belongs to $\operatorname{Iso}\left(X_{1}^{\Delta} \times \Omega_{1}, X_{2}^{\Delta} \times \Omega_{2}\right)$. The latter set of isomorphisms is defined as follows.

First consider an element $\boldsymbol{\kappa} \in \operatorname{Iso}\left(\mathbb{R} \times X_{1} \times \Omega_{1}, \mathbb{R} \times X_{2} \times \Omega_{2}\right)$ with the following properties:

(i) $\left.\boldsymbol{\kappa}\right|_{\overline{\mathbb{R}}_{+} \times X_{1} \times \Omega_{1}}: \overline{\mathbb{R}}_{+} \times X_{1} \times \Omega_{1} \rightarrow \overline{\mathbb{R}}_{+} \times X_{2} \times \Omega_{2}$ is a homeomorphism,

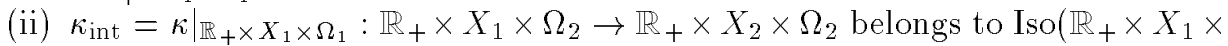
$\left.\Omega_{1}, \mathbb{R}_{+} \times X_{2} \times \Omega_{2}\right)$ and $\kappa_{0}=\left.\kappa\right|_{\{0\} \times X_{1} \times \Omega_{1}}:\{0\} \times X_{1} \times \Omega_{1} \rightarrow\{0\} \times X_{2} \times \Omega_{2}$ to $\operatorname{Iso}\left(\{0\} \times X_{1} \times \Omega_{1},\{0\} \times X_{2} \times \Omega_{2}\right)$,

(iii) The map $\delta: X_{1} \times \Omega_{1} \rightarrow X_{2} \times \Omega_{2}$ induced by $\kappa_{0}$ has the form $\delta\left(y_{1}\right)=$ $\left(\xi\left(y_{1}\right), \eta\left(y_{1}\right)\right)$ for a diffeomorphism $\eta: \Omega_{1} \rightarrow \Omega_{2}$ and an element $\xi \in$ $C^{\infty}\left(\Omega_{1}, \operatorname{Iso}\left(X_{1}, X_{2}\right)\right)$.

Then $\boldsymbol{\kappa} / \kappa_{0}: X_{1}^{\Delta} \times \Omega_{1} \rightarrow X_{2}^{\Delta} \times \Omega_{2}$ is a homeomorphism.

Now Iso $\left(X_{1}^{\Delta} \times \Omega_{1}, X_{2}^{\Delta} \times \Omega_{2}\right)$ is defined to be the set of all homeomorphisms that can be written in the form $\boldsymbol{\kappa} / \kappa_{0}$ for suitable $\boldsymbol{\kappa}$ with the properties (i), (ii) and (iii).

Moreover, $C^{\infty}\left(\Sigma, \operatorname{Iso}\left(X_{1}^{\Delta} \times \Omega_{1}, X_{2}^{\Delta} \times \Omega_{2}\right)\right)$ is the subset of all elements $\alpha \in$ $\operatorname{Iso}\left(X_{1}^{\Delta} \times \Omega_{1} \times \Sigma, X_{2}^{\Delta} \times \Omega_{2} \times \Sigma\right)$ such that $\left.\alpha\right|_{X_{1}^{\Delta} \times \Omega_{1} \times\{z\}}: X_{1}^{\Delta} \times \Omega_{1} \times\{z\} \rightarrow$ $X_{2}^{\Delta} \times \Omega_{2} \times\{z\}$ induces an element $\alpha(z) \in \operatorname{Iso}\left(X_{1}^{\Delta} \times \Omega_{1}, X_{2}^{\Delta} \times \Omega_{2}\right)$ for each $z \in \Sigma$.

We can arrange the manifolds with singularities as a category with an evident notion of morphisms. The simple details are left to the reader.

Note that condition (iii) allows us to identify $X_{1}$ and $X_{2}$ with realisations of a manifold $X$ with singularities; then the transition maps $\delta$ form a cocycle of an $X$-bundle on a corresponding edge.

Remark 1.1 For convenience we impose the condition that all occurring manifolds $M$ with (polyhedral) singularities define a chain of spaces $M \supset M^{\prime} \supset M^{\prime \prime} \supset \ldots \supset$ $M^{(j)} \supset \ldots \supset M^{(e)}, e=$ ord $M$, that ends with a closed compact $C^{\infty}$ manifold $M^{(e)}$ (the case $\operatorname{dim} M^{(e)}=0$ corresponds to a finite set of corner points, $\operatorname{dim} M^{(e)}>0$ to an edge). In other words, we exclude spaces like $M=\left\{x \in \mathbb{R}^{3}: x_{1}^{2}+x_{2}^{2}=x_{3}^{2}, 0 \leq\right.$ $\left.x_{3} \leq 1\right\}$ where $M^{\prime}=M^{(1)}$ equals $\{0\} \cup\left\{x \in \mathbb{R}^{3}: x_{1}^{2}+x_{2}^{2}=1, x_{3}=1\right\}$. Of course, our considerations easily generalise to such cases, though the formalities are more clumsy.

Parallel to the inductive way to define a manifold $M$ with polyhedral singularities we can introduce an associated stretched manifold $\mathbb{M}$. If $\chi: U \rightarrow X^{\Delta} \times \Omega$ is a singular chart on $M$, the corresponding stretched analogue for $\mathbb{M}$ is $\chi: \mathbb{U} \rightarrow \overline{\mathbb{R}}_{+} \times \mathbb{X} \times \Omega$, where $\mathbb{X}$ is the stretched base of the model cone and $\mathbb{U}$ the (invariantly defined) stretched analogue of $U$. In particular, if $X$ is a closed compact $C^{\infty}$ manifold and $M=X^{\Delta}$, we have $\mathbb{X}=X$, and $\mathbb{M}=\overline{\mathbb{R}}_{+} \times X$.

Remark 1.2 If $M$ is a manifold with singularities, we can introduce $\operatorname{Vect}(M)$, the set of all (complex) vector bundles on $M$ that are defined inductively, where $\operatorname{Vect}(M)$ for a $C^{\infty}$ manifold is defined to be the set of all complex $C^{\infty}$ vector bundles on $M$. Given $\operatorname{Vect}(N)$ for all manifolds with singularities for ord $N<\operatorname{ord} M$ we obtain any $E \in \operatorname{Vect}(M)$ by requiring $\left.E\right|_{M \backslash M^{\prime}} \in \operatorname{Vect}\left(M \backslash M^{\prime}\right)$, while $\left.E\right|_{V \backslash M^{\prime}}$ for every edge or corner neighbourhood $V \cong X^{\Delta} \times \Omega$ is the pull-back of a bundle on $\mathbb{R} \times X \times \Omega$ under $V \backslash M^{\prime} \rightarrow \mathbb{R}_{+} \times X \times \Omega$, and the transition maps of local representatives are restrictions of isomorphisms from $\mathbb{R} \times X \times \Omega$ to $\mathbb{R}+\times X \times \Omega$. In this way our vector bundles are, in fact, given on the corresponding stretched 
manifolds, and $M^{\prime} \hookrightarrow M$ induces a restriction $\operatorname{Vect}(M) \rightarrow \operatorname{Vect}\left(M^{\prime}\right)$. It is now straightforward how to introduce Riemannian metrics on $M$, Hermitian metrics in the bundles, and to define the spaces $C^{\infty}(M)$ of smooth functions on $M$ as well as $C_{0}^{\infty}(M)$, the subspace of functions with compact support. Let us recall once again that these notions refer to $\mathbb{M}$, though we write non-stretched objects in the brackets.

Note that there are many ways to introduce stratified spaces; the details very much depend on the analytic theory that is expected to hold on such spaces. Our definitions give a (relatively simple) background for sophisticated operator and symbol hierarchies that are non-trivial even on cones or on wedges with smooth base manifolds (links). In a talk of the (October 1999) Seminar "Analysis on Spaces with Singularities" (jointly organised by the University of Potsdam and the Humboldt University, Berlin) Pflaum of the Humboldt University presented elements of a Riemannian geometry on stratified spaces with transitions of singular charts and smoothness of functions that are more restricted than ours but motivated by other applications.

We will establish a program to synthesise operator algebras $\mathcal{L}(M)$ on $M$. Our procedure is briefly called conification and edgification of operator algebras $\mathcal{L}(N)$ that have been produced before for ord $N<$ ord $M$, and it is iterative. It starts from known operator algebras on spaces $N$ with ord $N \leq 1$. For the $C^{\infty}$ case ord $N=0$ we set $\mathcal{L}(N)=\bigcup_{\mu} L_{\text {cl }}^{\mu}(N)$, with $L_{c l}^{\mu}(N)$ being the space of classical pseudo-differential operators of order $\mu$ on $N$. For ord $N=1, N=\overline{\mathbb{R}}_{+}$, we define $\mathcal{L}\left(\overline{\mathbb{R}}_{+}\right)$to be the cone algebra on the half-axis as it may be found in [63], [66] (there are in fact various choices of cone algebras, $c f$. the discussion below and the paper of Lauter and Seiler [30]). The analytic descriptions are always given on stretched manifolds.

The conification and edgification process, as far as it is explicitly elaborated in papers and monographs, cf. [63], [66], and the references there, covers a number of interesting special cases. Examples are the algebras of boundary value problems (on $C^{\infty}$ manifolds with $C^{\infty}$ boundary) with the transmission property, cf. Boutet de Monvel [5], and without the transmission property, cf. Rempel and Schulze [44], [45]. These algebras can be regarded as edgifications of the corresponding boundary symbolic calculi, i.e., of pseudo-differential algebras on the half-axis (the inner normal), with the boundary as the edge. The standard boundary (and potential) conditions are generated within the formalism.

Other examples are classical operators of Fuchs type on a cone with a closed, compact $C^{\infty}$ manifold $X$ as base. Corresponding algebras can be interpreted as conifications of $\bigcup_{\mu} L_{c l}^{\mu}(X)$, cf. [58]. Boundary value problems on cones with compact $C^{\infty}$ bases $X$ with $C^{\infty}$ boundary are conifications of the above boundary value problems on $X$ itself, cf. Schrohe and Schulze [48], [49] for the case of operators with the transmission property. The corresponding cone algebra of boundary value problems contains the operators of Kondrat'ev's paper [25], together with their parametrices.

Pseudo-differential operators on manifolds with conical singularities have been studied by many authors under different aspects, cf. Plamenevskij [42], Melrose and Mendoza [38], and Schulze [54]. It is not the role of our discussion here to give a complete historical account. But it is worth observing that, although the different versions of cone pseudo-differential calculi meet (basically) the same Fuchs type symbols in the interior, the specific choices of ideals of smoothing operators 
enormously affect the higher calculi, including non-trivial contributions to the index theory in the elliptic case.

This concerns, in particular, the edgification of cone algebras on an infinite cone $X^{\Delta}$. It gives rise to edge problems for edge-degenerate operators, cf. Schulze [56], [63] or to edge boundary value problems, cf. Schrohe and Schulze [52], [51], [50]. Conifications of cone algebras yield algebras for corners of second order, cf. Schulze [59], etc. In each step we have some freedom in the choice of smoothing operators (that are in general not compact in weighted Sobolev spaces), and we get in this way a rich variety of higher algebras. In other words, higher edge and corner algebras, as far as they exist in the literature at all, are by no means determined by the (degenerate) symbols on $M \backslash M^{\prime}$ alone.

We can control to some extent this ambiguity, if we impose reasonable requirements. In particular, it is adequate to demand that the algebra $\mathcal{L}(M)$ that we achieve on $M$ contains $\mathcal{L}\left(M^{\prime}\right), \mathcal{L}\left(M^{\prime \prime}\right), \ldots$ on the "lower dimensional skeleta" $M^{\prime}$, $M^{\prime \prime}, \ldots$ as subalgebras. The simplest case for such a property is the algebra of boundary value problems on a (compact) $C^{\infty}$ manifold $X$ with $C^{\infty}$ boundary $X^{\prime}$ where $\mathcal{L}\left(X^{\prime}\right)$ equals $\bigcup_{\mu} L_{\text {cl }}^{\mu}\left(X^{\prime}\right)$ (involved vector bundles are omitted in this notation).

\section{Higher wedge theories}

We now turn to higher pseudo-differential wedge theories, using corresponding information from calculi on cones of lower order. Starting from an algebra on a cone $X^{\Delta}$ for a closed, compact $C^{\infty}$ base $X$, cf. [58], [72], we get in this way the wedge theory on $X^{\Delta} \times \Omega$, introduced in Schulze [56], cf. also [60], [63]. From this we want to read off the essential features to continue the iteration process.

Let $X=X^{(0)}$ be a compact manifold with singularities, and let $X^{\prime} \supset X^{\prime \prime} \supset$ $\ldots \supset X^{(e)}$ be the chain of singular subspaces in the sense of the previous section with a unique $e=$ ord $X$, such that $X^{(e)} \neq \emptyset, X^{(e+1)}=\emptyset$. Under our assumptions, cf. Remark 1.1, $X^{(e)}$ is a closed, compact $C^{\infty}$ manifold; for $\operatorname{dim} X^{(e)}=0$ it consists of a finite number of corner points of $X$.

Let $\left(\gamma_{1}, \ldots, \gamma_{e+1}\right)$ be a tuple of weights and $\gamma^{(j)}=\left(\gamma_{j}, \ldots, \gamma_{e+1}\right)$ for $1 \leq j \leq e+1$ and $\gamma^{(j)}-\mu=\left(\gamma_{j}-\mu, \ldots, \gamma_{e+1}-\mu\right)$ for any real $\mu$. Set $\gamma=\left(\gamma^{(j)}\right)_{1 \leq j \leq e+1}$ and $\gamma-\mu=\left(\gamma^{(j)}-\mu\right)_{1 \leq j \leq e+1}$. We shall often consider weight data $g=(\gamma, \gamma-\mu)$ that refer to the cone $X^{\Delta}$ with ord $X^{\Delta}=e+1$ and weight data $\boldsymbol{h}=(\beta, \beta-\mu)$ that refer to $X$ where $\beta=\left(\beta^{(k)}\right)_{1 \leq k \leq e}$ for $\beta^{(k)}=\left(\gamma_{k}, \ldots, \gamma_{e}\right), 1 \leq k \leq e$, and $\beta-\mu=\left(\beta^{(k)}-\mu\right)_{1 \leq k \leq e}$ for $\beta^{(k)}-\mu=\left(\gamma_{k}-\mu, \ldots, \gamma_{e}-\mu\right)$.

This is a special case of a more general notation

$$
\gamma^{(j)}=\left(\beta^{(j)}, \gamma_{e+1}\right)_{1 \leq j \leq e}, \quad \gamma^{(e+1)}=\gamma_{e+1},
$$

where $\beta^{(j)}$ (with $\beta^{(e+1)}=\emptyset$ ) is any tuple of already given weights for the axial variables in local representations of $X^{(j)}$ in stretched coordinates; $\beta^{(j)}$ is inductively generated and not necessarily explicitly specified.

The axial variables of cones will often be treated as $\mathbb{R}_{+}$-variables though the stretched spaces of the singular manifolds are defined in terms of $\overline{\mathbb{R}}_{+}$. We hope that this small inconsistency will not cause confusion.

The weights $\left(\gamma_{1}, \gamma_{2}, \ldots, \gamma_{e}, \gamma_{e+1}\right)$ are associated with $\left(r_{1}, r_{2}, \ldots, r_{e}, r_{e+1}\right) \in$ $\left(\mathbb{R}_{+}\right)^{e+1}$. The variable $r_{e+1}$ comes from a local representation of $X^{\Delta}$ as $\mathbb{R}_{+} \times V_{e}$, where $V_{e}$ is a neighbourhood of a point $x_{0} \in X$ of local form $\mathbb{R}_{+} \times \Omega_{e} \times V_{e-1} \ni$ 
$\left(r_{e}, y_{e}, x_{1}\right)$, and so on. To the chosen sequence of neighbourhoods $V_{e}, V_{e-1}, \ldots, V_{1}$, $x_{j} \in V_{e-j} \subset X^{(j)}$, we get stretched coordinates

$$
\begin{aligned}
& \left(r_{e+1}, r_{e}, y_{e}, r_{e-1}, y_{e-1}, \ldots, r_{1}, x\right) \\
& \quad \in \mathbb{R}_{+} \times\left(\mathbb{R}_{+} \times \Omega_{e} \times\left(\mathbb{R}_{+} \times \Omega_{e-1} \times\left(\ldots \times \mathbb{R}_{+} \times X^{(e)}\right)\right) \ldots\right) .
\end{aligned}
$$

The open sets $\Omega_{j}$ represent edges of dimensions $q_{j}$; by assumption $X^{(e)} \ni x$ is a closed, compact $C^{\infty}$ manifold, the base of the first cone (for convenience the numeration of the sets $\Omega_{j}$ in (2.2) is chosen in another way than in the preceding section). Clearly, the stretched coordinates depend on the chosen sequence of points $x_{j} \in X^{(j)}$ with $x_{j} \in V_{e-j}$. That means that the lower order weight information $\beta$ in general is a system of local weight information according to the considered region on $X$. This formal aspect does not influence the essential features of our theory, since it is inductive and because we always work with the highest weight $\gamma_{e+1}$ explicitly, while $\beta$ has been treated in the steps before. In order to avoid confusion with this purely combinatorial point the reader may always consider a model situation, e.g., take for $X$ the unit cube in $\mathbb{R}^{n}$, where this notation is absolutely transparent. For simplicity we do not discuss different weights for different corner points though this is, of course, possible.

The data on pseudo-differential algebras on cones $X^{(j) \Delta}:=\left(X^{(j)}\right)^{\Delta}, 0 \leq j \leq e$, that are used in this section will be referred to as $\mathbf{C} \boldsymbol{k}, k \in \mathbb{N}$. In Section 3 below they are produced in terms of various elements on pseudo-differential algebras on wedges of order $\leq e-1$, referred to as $\mathbf{W} \boldsymbol{j}, j \in \mathbb{N}$.

C0: There are scales of weighted Sobolev spaces $\mathcal{K}^{s, \gamma^{(j+1)}}\left(X^{(j) \Delta}, E^{j}\right), s \in \mathbb{R}$, $0 \leq j \leq e$ for vector bundles $E^{j} \in \operatorname{Vect}\left(X^{(j) \Delta}\right)$. The spaces are expressed with respect to a splitting of coordinates $\left(r, x_{(j)}\right) \in \mathbb{R}_{+} \times X^{(j)}$ (though invariant under isomorphisms of cones), with the property

$$
\mathcal{K}^{s,\left(\gamma_{j+1}, \ldots, \gamma_{e+1}\right)}\left(X^{(j) \Delta}, E^{j}\right)=k^{\gamma_{e+1}-\delta}(r) \mathcal{K}^{s,\left(\gamma_{j+1}, \ldots, \gamma_{e}, \delta\right)}\left(X^{(j) \Delta}, E^{j}\right)
$$

for arbitrary $\delta \in \mathbb{R}$. Here and in the sequel $r \in \mathbb{R}_{+}$is used in the meaning $r_{e+1}$. Here, $k^{\alpha}(r)$ for $\alpha \in \mathbb{R}$ is any strictly positive $C^{\infty}$ function on $\mathbb{R}_{+}$with $k^{\alpha}(r)=r^{\alpha}$ for $0<r<c_{0}, k^{\alpha}(r)=1$ for $c_{1}<r<\infty$, with constants $0<c_{0}<c_{1}$.

The precise nature of the spaces in $\mathrm{C} \mathbf{0}$ will become clear by Definition 3.2 below.

C1: There are fixed scalar products in the spaces $\mathcal{K}^{s, \gamma^{(j+1)}}\left(X^{(j) \Delta}, E^{j}\right)$. In particular, $\mathcal{K}^{0, \gamma^{(j+1)}}\left(X^{(j) \Delta}, E^{j}\right)$ for $\gamma^{(j+1)}=(0,0, \ldots, 0)$ serves as reference space that equals $L^{2}\left(X^{(j) \Delta}, E^{j}\right)$ (defined in terms of fixed metrics on the cones and Hermitian metrics in the bundles). On the space $\mathcal{K}^{s, \gamma^{(j+1)}}\left(X^{(j) \Delta}, E^{j}\right)$ we have a strongly continuous group of isomorphisms

$$
\kappa_{\lambda}^{(j)}: \mathcal{K}^{s, \gamma^{(j+1)}}\left(X^{(j) \Delta}, E^{j}\right) \rightarrow \mathcal{K}^{s, \gamma(j+1)}\left(X^{(j) \Delta}, E^{j}\right),
$$

$\lambda \in \mathbb{R}_{+}$, given by

$$
\left(\kappa_{\lambda}^{(j)} u\right)\left(r, x_{(j)}\right)=\lambda^{\frac{1}{2}\left(1+\operatorname{dim} X^{(j)}\right)} u\left(\lambda r, x_{(j)}\right),
$$

$\operatorname{dim} X^{(j)}:=\operatorname{dim}\left(X^{(j)} \backslash X^{(j+1)}\right)$.

Definition 2.1 Let $H$ and $\tilde{H}$ be Hilbert spaces, endowed with strongly continuous groups of isomorphisms $\left\{\kappa_{\lambda}\right\}_{\lambda \in \mathbb{R}_{+}}$and $\left\{\tilde{\kappa}_{\lambda}\right\}_{\lambda \in \mathbb{R}_{+}}$, respectively. Then $S^{\mu}(U \times$ 
$\left.\mathbb{R}^{m} ; H, \widetilde{H}\right)$ for $\mu \in \mathbb{R}, U \subseteq \mathbb{R}^{p}$ open, is defined to be the set of all $a(y, \eta) \in$ $C^{\infty}\left(U \times \mathbb{R}^{m}, \mathcal{L}(H, \widetilde{H})\right)$ such that

$$
\left\|\widetilde{\kappa}_{\langle\eta\rangle}^{-1}\left\{D_{y}^{\alpha} D_{\eta}^{\beta} a(y, \eta)\right\} \kappa\langle\eta\rangle\right\|_{\mathcal{L}(H, \tilde{H})} \leq c\langle\eta\rangle^{\mu-|\beta|}
$$

for all $\alpha \in \mathbb{N}^{p}, \beta \in \mathbb{N}^{m}, y \in K$ for arbitrary $K \subset U, \eta \in \mathbb{R}^{q}$, with constants $c=c(\alpha, \beta, K)>0,\langle\eta\rangle=\left(1+|\eta|^{2}\right)^{\frac{1}{2}}$.

$\mathcal{L}(H, \tilde{H})$ is the space of all linear continuous operators $H \rightarrow \widetilde{H}$ with the operator norm. In the case $H=\mathbb{C}^{N}$ we set $\kappa_{\lambda}=\mathrm{id}_{H}$ for all $\lambda \in \mathbb{R}_{+}$. Thus, for $H=\widetilde{H}=$ $\mathbb{C}$ we get the standard scalar symbol spaces. The operator-valued symbol spaces $S^{\mu}\left(U \times \mathbb{R}^{m} ; H, \tilde{H}\right)$ are systematically studied in [58], [63]. Elementary properties and results (on asymptotic sums, embeddings of symbol spaces, algebraic operations) as they are known for scalar symbols are valid for the operator-valued case in analogous form. $S^{\mu}\left(U \times \mathbb{R}^{m} ; H, \widetilde{H}\right)$ is a Fréchet space with the best constants in (2.5) as semi-norms.

Let $S^{(\mu)}\left(U \times\left(\mathbb{R}^{m} \backslash\{0\}\right) ; H, \tilde{H}\right)$ be the subspace of all $f(y, \eta) \in C^{\infty}\left(U \times\left(\mathbb{R}^{m} \backslash\right.\right.$ $\{0\}), \mathcal{L}(H, \widetilde{H}))$ that satisfy the relation

$$
f(y, \lambda \eta)=\lambda^{\mu} \widetilde{\kappa}_{\lambda} f(y, \eta) \kappa_{\lambda}^{-1}
$$

for all $\lambda \in \mathbb{R}_{+},(y, \eta) \in \mathbb{R}^{m} \backslash\{0\}$. The property (2.6) is also referred to as twisted homogeneity (with respect to $\left\{\kappa_{\lambda}\right\}_{\lambda \in \mathbb{R}_{+}}$and $\left\{\widetilde{\kappa}_{\lambda}\right\}_{\lambda \in \mathbb{R}_{+}}$). If $\chi(\eta)$ is an excision function (i.e., $\chi \in C^{\infty}\left(\mathbb{R}^{m}\right), \chi(\eta)=0$ for $0<|\eta|<c_{0}, \chi(\eta)=1$ for $c_{1}<|\eta|$ for certain $\left.0<c_{0}<c_{1}\right)$ we have $\chi(\eta) S^{(\mu)}\left(U \times\left(\mathbb{R}^{m} \backslash\{0\}\right) ; H, \widetilde{H}\right) \subset S^{\mu}\left(U \times \mathbb{R}^{m} ; H, \tilde{H}\right)$.

The space $S_{\mathrm{cl}}^{\mu}\left(U \times \mathbb{R}^{m} ; H, \widetilde{H}\right)$ of classical symbols is defined to be the set of all $a(y, \eta) \in S^{\mu}\left(U \times \mathbb{R}^{m} ; H, \widetilde{H}\right)$ that have an asymptotic expansion $a \sim \sum_{j=0}^{\infty} \chi a_{(\mu-j)}$ for certain $a_{(\mu-j)} \in S^{(\mu-j)}\left(U \times\left(\mathbb{R}^{m} \backslash\{0\}\right) ; H, \tilde{H}\right), j \in \mathbb{N}$. The space $S_{\text {cl }}^{\mu}(U \times$ $\mathbb{R}^{m} ; H, \widetilde{H}$ ) is Fréchet in a canonical way. In this topology (that is stronger than that induced by $\left.S^{\mu}\left(U \times \mathbb{R}^{m} ; H, \widetilde{H}\right)\right)$ the homogeneous components $a_{(\mu-j)}, j \in \mathbb{N}$, (that are uniquely determined by $a$ ) continuously depend on $a$. We set

$$
\sigma_{\Lambda}(a)(y, \eta)=a_{(\mu)}(y, \eta)
$$

for $(y, \eta) \in U \times\left(\mathbb{R}^{m} \backslash\{0\}\right)$, called the homogeneous principal symbol of $a$.

Let $\Omega \subset \mathbb{R}^{q}$ be an open set. An integral operator $(C u)(y)=\int c\left(y, y^{\prime}\right) u\left(y^{\prime}\right) d y^{\prime}$ with a kernel $c\left(y, y^{\prime}\right) \in C^{\infty}(\Omega \times \Omega, \mathcal{L}(H, \widetilde{H}))$, acting on $u \in C_{0}^{\infty}(\Omega, H)$, is called smoothing. Denote the space of all smoothing operators by $L^{-\infty}(\Omega ; H, \widetilde{H})$, equipped with a canonical Fréchet topology, and introduce

$$
\begin{aligned}
& L_{(\mathrm{cl})}^{\mu}(\Omega ; H, \widetilde{H})= \\
& \quad\left\{\mathrm{Op}(a)+C: a(y, \eta) \in S_{(\mathrm{cl})}^{\mu}\left(\Omega \times \mathbb{R}^{q} ; H, \widetilde{H}\right), C \in L^{-\infty}(\Omega ; H, \widetilde{H})\right\},
\end{aligned}
$$

the space of all pseudo-differential operators on $\Omega$ with operator-valued symbols (subscript (cl) means that we talk about classical or non-classical objects) where

$$
\mathrm{Op}(a) u(y)=\iint e^{i\left(y-y^{\prime}\right) \eta} a(y, \eta) u\left(y^{\prime}\right) d y^{\prime} d \eta,
$$

$d \eta=(2 \pi)^{-q} \mathrm{~d} \eta$. In place of Op we also write $\mathrm{Op}_{y}$. It can easily be verified that every $A \in L^{\mu}(\Omega ; H, \widetilde{H})$ induces a continuous operator $A: C_{0}^{\infty}(\Omega, H) \rightarrow C^{\infty}(\Omega, \widetilde{H})$. 
Pseudo-differential operators are also needed in parameter-dependent form. Set $U=\Omega \times \Sigma$ for open $\Omega \subseteq \mathbb{R}^{q}, \Sigma \subseteq \mathbb{R}^{l}$, with variables $(y, z)$ and covariables $(\eta, \zeta) \in$ $\mathbb{R}^{q+l}$. We then have the symbol spaces

$$
S_{(\mathrm{cl})}^{\mu}\left(\Omega \times \mathbb{R}^{q} ; H, \tilde{H} ; \Sigma \times \mathbb{R}^{l}\right):=S_{(\mathrm{cl})}^{\mu}\left(\Omega \times \Sigma \times \mathbb{R}^{q+l} ; H, \tilde{H}\right) .
$$

Observe that $a(y, \eta ; z, \zeta) \in S_{(\mathrm{cl})}^{\mu}\left(\Omega \times \mathbb{R}^{q} ; H, \tilde{H} ; \Sigma \times \mathbb{R}^{l}\right)$ implies $a\left(y, \eta ; z_{0}, \zeta_{0}\right) \in$ $S_{(\mathrm{cl})}^{\mu}\left(\Omega \times \mathbb{R}^{q} ; H, \widetilde{H}\right)$ for every fixed $\left(z_{0}, \zeta_{0}\right) \in \Sigma \times \mathbb{R}^{l}$.

Let $L^{-\infty}\left(\Omega ; H, \tilde{H} ; \Sigma \times \mathbb{R}^{l}\right)=C^{\infty}\left(\Sigma, \mathcal{S}\left(\mathbb{R}^{l}, L^{-\infty}(\Omega ; H, \tilde{H})\right)\right)$, and set

$$
\begin{aligned}
& L_{(\mathrm{cl})}^{\mu}\left(\Omega ; H, \widetilde{H} ; \Sigma \times \mathbb{R}^{l}\right)=\left\{\mathrm{Op}_{y}(a)(z, \zeta)+C(z, \zeta):\right. \\
& a(y, \eta ; z, \zeta) \in S_{(\mathrm{cl})}^{\mu}\left(\Omega \times \mathbb{R}^{q} ; H, \widetilde{H} ; \Sigma \times \mathbb{R}^{l}\right), \\
& \left.\quad C(z, \zeta) \in L^{-\infty}\left(\Omega ; H, \tilde{H} ; \Sigma \times \mathbb{R}^{l}\right)\right\} .
\end{aligned}
$$

(2.8) is the space of parameter-dependent pseudo-differential operators with operator-valued symbols. Note that $A(z, \zeta) \in L_{(\mathrm{cl})}^{\mu}\left(\Omega ; H, \tilde{H} ; \Sigma \times \mathbb{R}^{l}\right)$ implies the relation $A\left(z_{0}, \zeta_{0}\right) \in L_{(\mathrm{cl})}^{\mu}\left(\Omega \times \mathbb{R}^{q} ; H, \widetilde{H}\right)$ for every fixed $\left(z_{0}, \zeta_{0}\right) \in \Sigma \times \mathbb{R}^{l}$.

If an element $A \in L_{(\mathrm{cl})}^{\mu}\left(\Omega ; H, \widetilde{H} ; \Sigma \times \mathbb{R}^{l}\right)$ is written in the form $A=\mathrm{Op}_{y}(a)+C$, cf. (2.8), we call $a(y, \eta ; z, \zeta)$ a (parameter-dependent) left symbol. The map $A \rightarrow a$ induces an isomorphism

$$
\begin{aligned}
L_{(\mathrm{cl})}^{\mu}\left(\Omega ; H, \tilde{H} ; \Sigma \times \mathbb{R}^{l}\right) / L^{-\infty}\left(\Omega ; H, \tilde{H} ; \Sigma \times \mathbb{R}^{l}\right) \\
\quad \rightarrow S_{(\mathrm{cl})}^{\mu}\left(\Omega \times \mathbb{R}^{q} ; H, \tilde{H} ; \Sigma \times \mathbb{R}^{l}\right) / S^{-\infty}\left(\Omega \times \mathbb{R}^{q} ; H, \tilde{H} ; \Sigma \times \mathbb{R}^{l}\right) .
\end{aligned}
$$

Thus, every $A \in L_{\text {cl }}^{\mu}\left(\Omega ; H, \widetilde{H} ; \Sigma \times \mathbb{R}^{l}\right)$ has a (parameter-dependent) homogeneous principal symbol

$$
\sigma_{\Lambda}(A)(y, \eta ; z, \zeta):=a_{(\mu)}(y, \eta ; z, \zeta),
$$

where $\sigma_{\Lambda}(a)=a_{(\mu)} \in S^{(\mu)}\left((\Omega \times \Sigma) \times\left(\mathbb{R}^{q+l} \backslash\{0\}\right) ; H, \tilde{H}\right)$ is the principal symbol of $a$. For $A \in L_{\mathrm{cl}}^{\mu}(\Omega ; H, \tilde{H})$ the additional variables do not appear and we have $\sigma_{\Lambda}(A)(y, \eta)=\sigma_{\Lambda}(a)(y, \eta)=a_{(\mu)}(y, \eta)$, cf. $(2.7)$.

We do not formulate here all standard elements of the pseudo-differential calculus as they are known from the scalar case in an analogous form, i.e., for $H=\widetilde{H}=\mathbb{C}$. Let us only mention the following result.

Theorem 2.2 Let $\kappa: \Omega \rightarrow \widetilde{\Omega}$ be a diffeomorphism between open sets $\Omega, \widetilde{\Omega} \in \mathbb{R}^{q}$. Then the (pointwise) operator push-forward $\kappa_{*} A:=\left(\kappa^{*}\right)^{-1} A \kappa^{*}: C_{0}^{\infty}(\widetilde{\Omega}) \rightarrow C^{\infty}(\widetilde{\Omega})$ induces isomorphisms

$$
\kappa_{*}: L_{(\mathrm{cl})}^{\mu}\left(\Omega ; H, \widetilde{H} ; \Sigma \times \mathbb{R}^{l}\right) \rightarrow L_{(\mathrm{cl})}^{\mu}\left(\widetilde{\Omega} ; H, \widetilde{H} ; \Sigma \times \mathbb{R}^{l}\right) .
$$

According to (2.9) there is a transformation rule for left symbols (modulo smoothing symbols) that is analogous to the scalar one.

Definition 2.3 Let $H$ be a Hilbert space, equipped with a strongly continuous group $\left\{\kappa_{\lambda}\right\}_{\lambda \in \mathbb{R}_{+}}$of isomorphisms. Then $\mathcal{W}^{s}\left(\mathbb{R}^{q}, H\right), s \in \mathbb{R}$, is defined to be the completion of $\mathcal{S}\left(\mathbb{R}^{q}, H\right)$ (the Schwartz space of $H$-valued functions) with respect to the norm

$$
\left\{\int\langle\eta\rangle^{2 s}|| \kappa_{\langle\eta\rangle}^{-1}\left(F_{y \rightarrow \eta} u\right)(\eta) \|_{H}^{2} d \eta\right\}^{\frac{1}{2}} .
$$


The choice of $\left\{\kappa_{\lambda}\right\}_{\lambda \in \mathbb{R}_{+}}$is fixed and therefore not explicitly stressed by the notation. An exception is the case $\kappa_{\lambda}=\mathrm{id}_{H}$ for all $\lambda$; this yields the standard Sobolev space of $H$-valued distributions on $\mathbb{R}^{q}$, denoted by $H^{s}\left(\mathbb{R}^{q}, H\right)$. The spaces $\mathcal{W}^{s}\left(\mathbb{R}^{q}, H\right)$ have been introduced in [56]. The basic functional analytic properties may be found in the monographs [58] or [63], cf. also [22]. For an open set $\Omega \subseteq \mathbb{R}^{q}$ we have the comp and loc versions $\mathcal{W}_{\text {comp }}^{s}(\Omega, H)$ and $\mathcal{W}_{\text {loc }}^{s}(\Omega, H)$, similarly to the scalar case $\kappa_{\lambda}=$ id on $H=\mathbb{C}$. The spaces $\mathcal{W}^{s}\left(\mathbb{R}^{q}, H\right), \mathcal{W}_{\text {comp }}^{s}(\Omega, H)$ or $\mathcal{W}_{\text {loc }}^{s}(\Omega, H)$ are called abstract edge Sobolev spaces.

A simple example is $H=H^{s}\left(\mathbb{R}^{n}\right)$, the standard Sobolev space in $\mathbb{R}^{n}$ of smoothness $s$, endowed with $\left(\kappa_{\lambda} u\right)(x)=\lambda^{n / 2} u(\lambda x), \lambda \in \mathbb{R}_{+}$. In this case we have $\mathcal{W}^{s}\left(\mathbb{R}^{q}, H^{s}\left(\mathbb{R}^{n}\right)\right)=H^{s}\left(\mathbb{R}^{q} \times \mathbb{R}^{n}\right)$.

For purposes below it is also useful to have parameter-dependent norms with an additional parameter $\zeta \in \mathbb{R}^{l}$. We set

$$
\|u\|_{\mathcal{W}_{\zeta}^{s}\left(\mathbb{R}^{q}, H\right)}=\left\{\int\langle\eta, \zeta\rangle^{2 s}\left\|\kappa_{\langle\eta, \zeta\rangle}^{-1}\left(F_{y \rightarrow \eta} u\right)(\eta)\right\|_{H}^{2} d \eta\right\}^{\frac{1}{2}}
$$

Remark 2.4 The definition of pseudo-differential operators with operator-valued symbols as well as of abstract edge Sobolev spaces can easily be generalised to Fréchet spaces $H, \widetilde{H}$ that are written as projective limits of Hilbert spaces with group actions that are the same (up to extensions or restrictions) on all spaces of the scales. The obvious details are tacitly used below, cf. also [63].

Remark 2.5 There is also a version of edge Sobolev spaces with $\mathbb{R}_{+}$in place of $\mathbb{R}^{q}$ and the Mellin transform $M$ instead of the Fourier transform, $(M g)(w)=$ $\int_{0}^{\infty} r^{w-1} g(r) d r$. The completion of $C_{0}^{\infty}\left(\mathbb{R}_{+}, H\right)$ with respect to the norm

$$
\left\{\frac{1}{2 \pi i} \int_{\Gamma_{\frac{\nu+1}{2}-\beta}}\langle\operatorname{Im} w\rangle^{2 s}\left\|\kappa_{\langle\operatorname{Im} w\rangle}^{-1}(M g)(w)\right\|_{H}^{2} d w\right\}^{\frac{1}{2}}
$$

is denoted by $\mathcal{V}^{s, \beta}\left(\mathbb{R}_{+}, H\right), s, \beta \in \mathbb{R}$. The number $\nu \in \mathbb{N}$ is given in connection with $H$, i.e., $\nu=\nu(H)$, that it is assumed to be known in concrete cases. In particular, if $H$ is a Sobolev space on a cone with base $X^{(j)}$, we set $\nu=\operatorname{dim}\left(X^{(j)} \backslash X^{(j+1)}\right)$. Note that $\mathcal{V}^{s, \beta}\left(\mathbb{R}_{+}, H\right) \subset \mathcal{W}_{\mathrm{loc}^{s}}\left(\mathbb{R}_{+}, H\right)$, and we have $\mathcal{V}^{s, \beta+\delta}\left(\mathbb{R}_{+}, H\right)=r^{\delta} \mathcal{V}^{s, \beta}\left(\mathbb{R}_{+}, H\right)$ for arbitrary $\beta, \delta \in \mathbb{R}$.

Finally, we have a mixture between weighted Mellin and Fourier wedge Sobolev spaces $\mathcal{V}^{s, \beta}\left(\mathbb{R}_{+} \times \mathbb{R}^{q}, H\right)$, defined to be the completion of $C_{0}^{\infty}\left(\mathbb{R}_{+} \times \mathbb{R}^{q}, E\right)$ with respect to the norm

$$
\left\{\int_{\mathbb{R}^{q}} \frac{1}{2 \pi i} \int_{\Gamma_{\frac{\nu+q+1}{2}-\beta}}\langle\operatorname{Im} w, \eta\rangle^{2 s}\left\|\kappa_{\langle\operatorname{Im} w, \eta\rangle}^{-1}\left(M_{r \rightarrow w} F_{y \rightarrow \eta} g\right)(w, \eta)\right\|_{H}^{2} d w d \eta\right\}^{\frac{1}{2}}
$$

We also employ $\mathcal{V}^{s, \beta}\left(\mathbb{R}_{+}, H\right)$ for Fréchet spaces $H$, similarly to Remark 2.4 .

Theorem 2.6 Every $A \in L^{\mu}(\Omega ; H, \widetilde{H})$ induces a continuous operator

$$
A: \mathcal{W}_{\mathrm{comp}}^{s}(\Omega, H) \rightarrow \mathcal{W}_{\mathrm{loc}}^{s-\mu}(\Omega, \tilde{H})
$$

for every $s \in \mathbb{R}$.

For a proof, cf. [63], Theorem 1.3.69. 
Remark 2.7 The spaces of pseudo-differential operators with operator-valued symbols as well as the abstract wedge spaces can easily be defined on a $C^{\infty}$ manifold $\Omega$; then, in particular, Theorem 2.6 also holds globally on $\Omega$.

We shall employ a slight generalisation of the formalism in the sense that the spaces $H$ and $\widetilde{H}$ are of the form

$$
\boldsymbol{H}=\bigoplus_{j=0}^{e} H^{(j)}, \quad \widetilde{\boldsymbol{H}}=\bigoplus_{k=0}^{e} \widetilde{H}^{(k)}
$$

where the spaces $H^{(j)}$ and $\widetilde{H}^{(k)}$ are endowed with group actions $\left\{\kappa_{\lambda}\right\}_{\lambda \in \mathbb{R}_{+}}=$ $\operatorname{diag}\left\{\kappa_{\lambda}^{(j)}\right\}_{\lambda \in \mathbb{R}_{+}}$and $\left\{\widetilde{\kappa}_{\lambda}\right\}_{\lambda \in \mathbb{R}_{+}}=\operatorname{diag}\left\{\widetilde{\kappa}_{\lambda}^{(k)}\right\}_{\lambda \in \mathbb{R}_{+}}$, respectively. We then have the symbol spaces

$$
S_{(\mathrm{cl})}^{\mu_{k j}}\left(\Omega \times \mathbb{R}^{q} ; H^{(j)}, \tilde{H}^{(k)} ; \Sigma \times \mathbb{R}^{l}\right)
$$

with certain orders $\mu_{k j} \in \mathbb{R}$ and associated operator spaces

$$
L_{(\mathrm{cl})}^{\mu_{k j}}\left(\Omega \times \mathbb{R}^{q} ; H^{(j)}, \tilde{H}^{(k)} ; \Sigma \times \mathbb{R}^{l}\right) .
$$

In our applications we have $\mu_{k j}=\mu-\frac{1}{2}\left(\delta_{j}-\delta_{k}\right)$ for some $\mu \in \mathbb{R}$ and a given tuple $\left(\delta_{0}, \ldots, \delta_{e}\right) \in Z^{e+1}$. Since the choice of $\left(\delta_{j}\right)_{j=0, \ldots, e}$ is fixed and known in every concrete case, we employ the notation

$$
\begin{aligned}
& \boldsymbol{S}_{(\mathrm{cl})}^{\mu}\left(\Omega \times \mathbb{R}^{q}, \boldsymbol{H}, \widetilde{\boldsymbol{H}} ; \Sigma \times \mathbb{R}^{l}\right)=\left\{a=\left(a_{k j}\right)_{0 \leq k, j \leq e}:\right. \\
& \left.\quad a_{k j} \in S_{\mathrm{cl}}^{\mu_{k j}}\left(\Omega \times \mathbb{R}^{q} ; H^{(j)}, \widetilde{H}^{(k)} ; \Sigma \times \mathbb{R}^{l}\right) \text { for } j, k=0, \ldots, e\right\}, \\
& \boldsymbol{L}_{(\mathrm{cl})}^{\mu}\left(\Omega ; \boldsymbol{H}, \widetilde{\boldsymbol{H}} ; \Sigma \times \mathbb{R}^{l}\right)=\left\{\mathcal{A}=\left(A_{k, j}\right)_{0 \leq k, j \leq e}:\right. \\
& \left.\quad A_{k j} \in L_{(\mathrm{cl})}^{\mu_{k j}}\left(\Omega \times \mathbb{R}^{q} ; H^{(j)}, \widetilde{H}^{(k)} ; \Sigma \times \mathbb{R}^{l}\right) \text { for } j, k=0, \ldots, e\right\} .
\end{aligned}
$$

Concerning homogeneity we set

$$
\begin{aligned}
& \boldsymbol{S}^{(\mu)}\left(\Omega \times\left(\mathbb{R}^{q} \backslash\{0\}\right), \boldsymbol{H}, \widetilde{\boldsymbol{H}}\right)=\left\{f(y, \eta)=\left(f_{k j}(y, \eta)\right)_{0 \leq k, j \leq e}:\right. \\
& \left.f_{k j}(y, \eta) \in S^{\left(\mu_{k j}\right)}\left(\Omega \times\left(\mathbb{R}^{q} \backslash\{0\}\right) ; H^{(j)}, \widetilde{H}^{(k)}\right) \text { for } 0 \leq k, j \leq e\right\},
\end{aligned}
$$

To express homogeneity, in analogy to relation (2.6) for $f(y, \eta) \in S^{(\mu)}\left(\Omega \times\left(\mathbb{R}^{q} \backslash\right.\right.$ $\{0\}), \boldsymbol{H}, \widetilde{\boldsymbol{H}})$ we write

$$
f(y, \lambda \eta)=\{\lambda\}^{\mu} \widetilde{\kappa}_{\lambda} f(y, \eta) \kappa_{\lambda}^{-1}
$$

for $\lambda \in \mathbb{R}_{+},(y, \eta) \in \Omega \times\left(\mathbb{R}^{q} \backslash\{0\}\right)$. Similar notation is used for the case with dependence on $(y, z, \eta \zeta) \in \Omega \times \Sigma \times\left(\mathbb{R}^{q+l} \backslash\{0\}\right)$.

Together with (2.11) we also consider the spaces

$$
H \oplus \mathbb{C}^{N_{-}} \quad \tilde{H} \oplus \mathbb{C}^{N_{+}}
$$

with certain $N_{-}, N_{+} \in \mathbb{N}$ and group actions $\operatorname{diag}\left(\left\{\kappa_{\lambda}^{(j)}\right\}_{\lambda \in \mathbb{R}_{+}}, \mathrm{id}_{\mathbb{C}^{N_{-}}}\right)$and $\operatorname{diag}\left(\left\{\widetilde{\kappa}_{\lambda}^{(k)}\right\}_{\lambda \in \mathbb{R}_{+}}, \operatorname{id}_{\mathbb{C}^{N_{+}}}\right)$, respectively. In this case $\left(\delta_{0}, \ldots, \delta_{e}\right)$ will be replaced by $\left(\delta_{0}, \ldots, \delta_{e}, 0\right)$, and we write instead of $(2.13)$

$$
\boldsymbol{L}_{(\mathrm{cl})}^{\mu}\left(\Omega ; \boldsymbol{H} \oplus \mathbb{C}^{N_{-}}, \widetilde{\boldsymbol{H}} \oplus \mathbb{C}^{N_{+}} ; \Sigma \times \mathbb{R}^{l}\right)
$$

with orders $\mu_{k j}=\mu-\frac{1}{2}\left(\delta_{j}-\delta_{k}\right), i, j=0, \ldots, e+1$; a similar notation is used for the corresponding symbol spaces, cf. (2.12). 
Every $\mathcal{A} \in \boldsymbol{L}_{(\mathrm{cl})}^{\mu}\left(\Omega ; \boldsymbol{H} \oplus \mathbb{C}^{N_{-}}, \widetilde{\boldsymbol{H}} \oplus \mathbb{C}^{N_{+}} ; \Sigma \times \mathbb{R}^{q}\right)$ represents a family of continuous maps

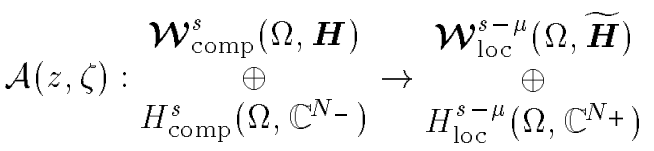

where

$$
\mathcal{W}_{\text {comp }}^{s}(\Omega, \boldsymbol{H}):=\bigoplus_{k=0}^{e} \mathcal{W}_{\text {comp }}^{s-\frac{1}{2} \delta_{j}}\left(\Omega, H^{(j)}\right)
$$

and analogously with subscript loc. It is clear that simple reductions of orders in terms of multiplication by diagonal block matrices from both sides connect classes $\boldsymbol{L}_{(\mathrm{cl})}^{\mu}$ with a different choice of $\left(\delta_{j}\right)$. However, for the applications below we prefer numbers $\left(\delta_{j}\right)$ of the form $\delta_{j}=\operatorname{dim} X^{(0)}-\operatorname{dim} X^{(j)}, j=0, \ldots, e$, when we discuss operators on an infinite cone or an infinite wedge; for future reference we will talk about DN-orders (from a scheme of orders according to Douglis-Nirenberg systems). Otherwise, for compact base spaces $X$ we often refer to the same orders in all components, cf. Remark 2.8 below, though DN-orders are possible as well, according to the chain of spaces $X=X^{(0)} \supset X^{(1)} \supset \ldots$

Let us now return to information from the calculus on a cone, $\mathrm{cf}$. C1. Let ord $X=$ $e$, set $\boldsymbol{X}^{\Delta}=\left(X^{(j) \Delta}\right)_{0 \leq j \leq e}, \boldsymbol{E}=\left(E^{j}\right)_{0 \leq j \leq e}$, and form the spaces

$$
\begin{aligned}
\mathcal{K}^{s, \gamma}\left(\boldsymbol{X}^{\Delta}, \boldsymbol{E}\right) & =\bigoplus_{j=0}^{e} \mathcal{K}^{s-\frac{1}{2} \delta_{j}, \gamma^{(j+1)}-\frac{1}{2} \delta_{j}}\left(X^{(j) \Delta}, E^{j}\right), \\
\mathcal{K}^{s^{*}, \gamma^{*}}\left(\boldsymbol{X}^{\Delta}, \boldsymbol{E}\right) & =\bigoplus_{j=0}^{e} \mathcal{K}^{s+\frac{1}{2} \delta_{j}, \gamma^{(j+1)}+\frac{1}{2} \delta_{j}}\left(X^{(j) \Delta}, E^{j}\right),
\end{aligned}
$$

endowed with $\left\{\kappa_{\lambda}\right\}_{\lambda \in \mathbb{R}_{+}}=\operatorname{diag}\left\{\kappa_{\lambda}^{(j)}\right\}_{\lambda \in \mathbb{R}_{+}}$, where $\delta_{j}=\operatorname{dim} X^{(0)}-\operatorname{dim} X^{(j)}, j=$ $0, \ldots, e$.

Note that there is a non-degenerate sesquilinear pairing between $\bigoplus_{j=0}^{e} \mathcal{K}^{s-\frac{1}{2} \delta_{j}, \gamma^{(j+1)}-\frac{1}{2} \delta_{j}}\left(X^{(j) \Delta}, E^{j}\right)$ and $\bigoplus_{j=0}^{e} \mathcal{K}^{-s+\frac{1}{2} \delta_{j},-\gamma^{(j+1)}+\frac{1}{2} \delta_{j}}\left(X^{(j) \Delta}, E^{j}\right)$ via the $\bigoplus_{j=0}^{e} \mathcal{K}^{0,0}\left(X^{(j) \Delta}, E^{j}\right)$-scalar product. This gives rise to a corresponding pairing between $\mathcal{K}^{s, \gamma}\left(\boldsymbol{X}^{\Delta}, \boldsymbol{E}\right)$ and $\mathcal{K}^{(-s)^{*},(-\gamma)^{*}}\left(\boldsymbol{X}^{\Delta}, \boldsymbol{E}\right)$.

Moreover, define the Fréchet space

$$
\mathcal{S}^{\gamma}\left(\boldsymbol{X}^{\Delta}, \boldsymbol{E}\right)=\lim _{\leftarrow} \in \mathbb{N}\left\{\langle r\rangle^{-N} \boldsymbol{K}^{N, \gamma}\left(\boldsymbol{X}^{\Delta}, \boldsymbol{E}\right)\right\} .
$$

Note that the group $\left\{\kappa_{\lambda}\right\}_{\lambda \in \mathbb{R}_{+}}$acts in the spaces on the right of (2.18) for every $N$. So we can talk about operator-valued symbols also for such spaces.

Incidentally, if we employ standard orders (not DN-orders), we refer to spaces of the form $(2.16)$ with $\delta_{j}=0$ for all $j$. The corresponding spaces will be denoted by

$$
\mathcal{K}^{s, \gamma}\left(\boldsymbol{X}^{\Delta}, \boldsymbol{E}\right)
$$

W0: The space $\mathcal{L}^{\mu}\left(X^{\Delta} \times \Omega, \boldsymbol{g} ;\left(\boldsymbol{E}, N_{-} ; \tilde{\boldsymbol{E}}, N_{+}\right) ; \Sigma \times \mathbb{R}^{l}\right)$ of (parameter-dependent) pseudo-differential operators on a wedge $X^{\Delta} \times \Omega$ (with edge $\Omega$ and model cone $X^{\Delta}$, with $X=X^{(0)}$ in the above notation) is a subspace of

$$
\bigcap_{s \in \mathbb{R}} \boldsymbol{L}^{\mu}\left(\Omega ; \mathcal{K}^{s, \gamma}\left(\boldsymbol{X}^{\Delta}, \boldsymbol{E}\right) \oplus \mathbb{C}^{N_{-}}, \mathcal{K}^{s-\mu, \gamma-\mu}\left(\boldsymbol{X}^{\Delta}, \tilde{\boldsymbol{E}}\right) \oplus \mathbb{C}^{N_{+}} ; \Sigma \times \mathbb{R}^{l}\right)
$$


Here $\boldsymbol{E}=\left(E^{j}\right)_{0 \leq j \leq e}$ and $\widetilde{\boldsymbol{E}}=\left(\widetilde{E}^{j}\right)_{0 \leq j \leq e}$ are tuples of vector bundles, $N_{+}\left(N_{-}\right) \in \mathbb{N}$ the number of trace (potential) conditions with respect to the edge $\Omega$, and $\boldsymbol{g}=$ $(\gamma, \gamma-\mu)$ are the involved weight data.

In other words, such operators have the form of block matrices

$$
\mathcal{A}(z, \zeta): \begin{gathered}
\mathcal{W}_{\mathrm{comp}}^{s}\left(\Omega, \mathcal{K}^{s, \gamma}\left(\boldsymbol{X}^{\Delta}, \boldsymbol{E}\right)\right) \\
H_{\mathrm{comp}}^{s}\left(\Omega, \mathbb{C}^{N_{-}}\right)
\end{gathered} \rightarrow \stackrel{\mathcal{W}_{\mathrm{loc}}^{s-\mu}\left(\Omega, \mathcal{K}^{s-\mu, \gamma-\mu}\left(\boldsymbol{X}^{\Delta}, \widetilde{\boldsymbol{E}}\right)\right)}{\rightarrow} \underset{H_{\mathrm{loc}}^{s-\mu}\left(\Omega, \mathbb{C}^{N_{+}}\right)}{\oplus}
$$

We will simply talk about (parameter-dependent) edge operators.

The details on the operator-valued symbols for the operators in $\mathbf{W} \mathbf{0}$ are given in C3 and W2 below. The operators themselves are defined in formula (2.22), where we employ the following class of smoothing operators:

W1: The space $\mathcal{L}^{-\infty}\left(X^{\Delta} \times \Omega, \boldsymbol{g} ;\left(\boldsymbol{E}, N_{-} ; \widetilde{\boldsymbol{E}}, N_{+}\right) ; \Sigma \times \mathbb{R}^{l}\right)$ is defined to be the set of all families of continuous operators

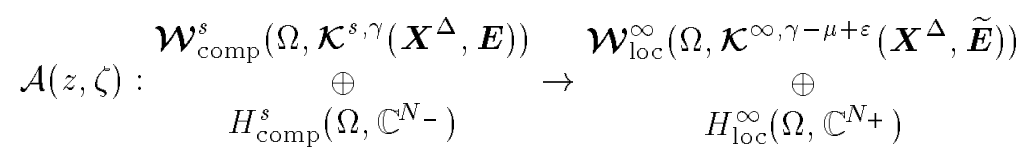

such that the (pointwise) formal adjoints (with respect to the above-mentioned scalar products) are continuous in the sense

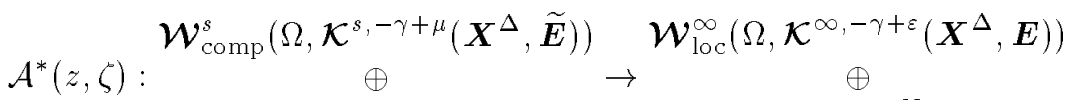

$$
\begin{aligned}
& H_{\text {comp }}^{s}\left(\Omega, \mathbb{C}^{N_{+}}\right) \quad H_{\mathrm{loc}}^{\infty}\left(\Omega, \mathbb{C}^{N_{-}}\right)
\end{aligned}
$$

for all $s \in \mathbb{R}$ (for an $\varepsilon>0$ which may depend on $\mathcal{A})$, where $\mathcal{A}(z, \zeta)$ and $\mathcal{A}^{*}(z, \zeta)$ is $C^{\infty}$ in $z \in \Sigma$ and a Schwartz function in $\zeta \in \mathbb{R}^{l}$, with values in the respective spaces of operators, $s \in \mathbb{R}$.

C2: On $X^{\Delta}$ we have a space of cone pseudo-differential operators $\mathcal{C}^{\mu}\left(X^{\Delta}, \boldsymbol{g} ; \boldsymbol{v}\right)$, of order $\mu \in \mathbb{R}$ with $\boldsymbol{v}=(\boldsymbol{E}, \widetilde{\boldsymbol{E}})$ and weight data $\boldsymbol{g}=(\gamma, \gamma-\mu), \gamma \in \mathbb{R}$, which is contained in $\bigcap_{s \in \mathbb{R}} \mathcal{L}\left(\mathcal{K}^{s, \gamma}\left(\boldsymbol{X}^{\Delta}, \boldsymbol{E}\right), \mathcal{K}^{s-\mu, \gamma-\mu}\left(\boldsymbol{X}^{\Delta}, \tilde{\boldsymbol{E}}\right)\right)$. There is a subspace $\mathcal{C}_{G}\left(X^{\Delta}, \boldsymbol{g} ; \boldsymbol{v}\right)$ of so-called Green operators; for every $G \in \mathcal{C}_{G}\left(X^{\Delta}, \boldsymbol{g} ; \boldsymbol{v}\right)$ there is an $\varepsilon>0$, such that

$$
\begin{aligned}
G & : \mathcal{K}^{s, \gamma}\left(\boldsymbol{X}^{\Delta}, \boldsymbol{E}\right) \rightarrow \mathcal{S}^{\gamma-\mu+\varepsilon}\left(\boldsymbol{X}^{\Delta}, \widetilde{\boldsymbol{E}}\right), \\
G^{*} & : \mathcal{K}^{s,(-\gamma+\mu)^{*}}\left(\boldsymbol{X}^{\Delta}, \widetilde{\boldsymbol{E}}\right) \rightarrow \mathcal{S}^{(-\mu)^{*}+\varepsilon}\left(\boldsymbol{X}^{\Delta}, \boldsymbol{E}\right)
\end{aligned}
$$

are continuous for all $s \in \mathbb{R} ; G^{*}$ is the formal adjoint of $G$ with respect to the above scalar product.

C3: There is a subspace $\boldsymbol{\mathcal { R }}^{\mu}\left(\Omega \times \mathbb{R}^{q}, \boldsymbol{g} ; \boldsymbol{w}\right), \boldsymbol{w}=\left(\boldsymbol{E}, N_{-} ; \widetilde{\boldsymbol{E}}, N_{+}\right)$, of

$$
\bigcap_{s \in \mathbb{R}} \boldsymbol{S}^{\mu}\left(\Omega \times \mathbb{R}^{q} ; \mathcal{K}^{s, \gamma}\left(\boldsymbol{X}^{\Delta}, \boldsymbol{E}\right) \oplus \mathbb{C}^{N_{-}}, \mathcal{K}^{s-\mu, \gamma-\mu}\left(\boldsymbol{X}^{\Delta}, \tilde{\boldsymbol{E}}\right) \oplus \mathbb{C}^{N_{+}}\right),
$$

where for $a(y, \eta)=\left(a_{i j}(y, \eta)\right)_{i, j=1,2} \in \mathcal{R}^{\mu}\left(\Omega \times \mathbb{R}^{q}, \boldsymbol{g} ; \boldsymbol{w}\right)$ we have $a_{11}\left(y_{0}, \eta_{0}\right) \in$ $\mathcal{C}^{\mu}\left(X^{\Delta}, \boldsymbol{g} ; \boldsymbol{v}\right)$ for every $\left(y_{0}, \eta_{0}\right) \in \Omega \times \mathbb{R}^{q}$, while

$$
\begin{aligned}
\left(\begin{array}{cc}
0 & a_{12} \\
a_{21} & a_{22}
\end{array}\right)(y, \eta) \in \bigcap_{s \in \mathbb{R}} \boldsymbol{S}_{\mathrm{cl}}^{\mu}\left(\Omega \times \mathbb{R}^{q} ; \mathcal{K}^{s, \gamma}\left(\boldsymbol{X}^{\Delta}, \boldsymbol{E}\right) \oplus \mathbb{C}^{N_{-}},\right. \\
\left.\mathcal{S}^{\gamma-\mu+\varepsilon}\left(\boldsymbol{X}^{\Delta}, \widetilde{\boldsymbol{E}}\right) \oplus \mathbb{C}^{N^{+}}\right),
\end{aligned}
$$




$$
\begin{aligned}
\left(\begin{array}{cc}
0 & a_{12} \\
a_{21} & a_{22}
\end{array}\right)^{*}(y, \eta) \in \bigcap_{s \in \mathbb{R}} \boldsymbol{S}_{\mathrm{cl}}^{\mu}\left(\Omega \times \mathbb{R}^{q} ; \mathcal{K}^{s,(-\gamma+\mu)^{*}}\left(\boldsymbol{X}^{\Delta}, \widetilde{\boldsymbol{E}}\right) \oplus \mathbb{C}^{N_{+}},\right. \\
\left.\boldsymbol{S}^{(-\gamma)^{*}+\varepsilon}\left(\boldsymbol{X}^{\Delta}, \boldsymbol{E}\right) \oplus \mathbb{C}^{N_{-}}\right)
\end{aligned}
$$

for some $\varepsilon>0$ (dependent on the operators).

Set

$$
\mathcal{R}^{\mu}\left(\Omega \times \Sigma \times \mathbb{R}^{q+l}, \boldsymbol{g} ; \boldsymbol{w}\right)=: \boldsymbol{\mathcal { R }}^{\mu}\left(\Omega \times \mathbb{R}^{q}, \boldsymbol{g} ; \boldsymbol{w} ; \Sigma \times \mathbb{R}^{l}\right)
$$

for $\boldsymbol{w}=\left(\boldsymbol{E}, N_{-} ; \tilde{\boldsymbol{E}}, N_{+}\right)$. Here, $(y, \eta) \in \Omega \times \mathbb{R}^{q}$ are regarded as variables and covariables and $(z, \zeta) \in \Sigma \times \mathbb{R}^{l}$ as parameters. The space of upper left corners of the block matrices (i.e., where $N_{-}=N_{+}=0$ ) is denoted by

$$
\boldsymbol{R}^{\mu}\left(\Omega \times \mathbb{R}^{q}, \boldsymbol{g} ; \boldsymbol{v} ; \Sigma \times \mathbb{R}^{l}\right), \quad \text { for } \boldsymbol{v}=(\boldsymbol{E}, \tilde{\boldsymbol{E}}),
$$

cf. the description in $\mathbf{W} \mathbf{2}$ below.

The operator space of Wo has the following structure:

$$
\begin{array}{r}
\mathcal{L}^{\mu}\left(X^{\Delta} \times \Omega, \boldsymbol{g} ; \boldsymbol{w} ; \Sigma \times \mathbb{R}^{l}\right) \\
=\left\{\omega \mathrm{Op}(a)(z, \zeta) \tilde{\omega}+(1-\omega) \mathcal{A}_{\text {int }}(z, \zeta)(1-\widetilde{\widetilde{\omega}})+\mathcal{C}(z, \zeta):\right. \\
a(y, \eta ; z, \zeta) \in \mathcal{R}^{\mu}\left(\Omega \times \mathbb{R}^{q}, \boldsymbol{g} ; \boldsymbol{w} ; \Sigma \times \mathbb{R}^{l}\right), \\
\mathcal{A}_{\text {int }}(z, \zeta) \in \mathcal{L}^{\mu}\left(\mathbb{R}_{+} \times X \times \Omega, \boldsymbol{h} ; \boldsymbol{w} ; \Sigma \times \mathbb{R}^{l}\right), \\
\left.\quad \mathcal{C}(z, \zeta) \in \mathcal{L}^{-\infty}\left(X^{\Delta} \times \Omega, \boldsymbol{g} ; \boldsymbol{w} ; \Sigma \times \mathbb{R}^{l}\right)\right\},
\end{array}
$$

where $\mu \in \mathbb{R}, \boldsymbol{g}=(\gamma, \gamma-\mu), \boldsymbol{w}=\left(\boldsymbol{E}, N_{-} ; \widetilde{\boldsymbol{E}}, N_{+}\right)$, and $\omega, \widetilde{\omega}, \widetilde{\widetilde{\omega}}$ are cut-off functions (i.e., elements in $C_{0}^{\infty}\left(\overline{\mathbb{R}}_{+}\right)$that equal 1 near $r=0$ ) with $\omega \widetilde{\omega}=\omega$, $\omega \widetilde{\tilde{\omega}}=\widetilde{\tilde{\omega}}$. Let

$$
\boldsymbol{L}^{\mu}\left(X^{\Delta} \times \Omega, \boldsymbol{g} ; \boldsymbol{v} ; \Sigma \times \mathbb{R}^{l}\right), \quad \boldsymbol{v}=(\boldsymbol{E}, \widetilde{\boldsymbol{E}}),
$$

be the space of upper left corners. The operator families $\mathcal{A}_{\text {int }}(z, \zeta)$ belong to an already established space $\mathbb{R}_{+} \times X \times \Omega$, that is is of lower singularity order, cf. C4 below. The weight data $\boldsymbol{h}$ are induced by $\boldsymbol{g}$ by omitting the components belonging to the tip of $X^{\Delta}$.

The iterative procedure gives us the following information from the steps before:

C4: On each compact manifold $M$ with singularities, ord $M=e$ for every $e \geq 0$, less than a given order, the following objects are known:

(i) Scales of weighted Sobolev spaces $\mathcal{H}^{s, \beta}(\boldsymbol{M}, \boldsymbol{F})=\bigoplus_{j=0}^{e} \mathcal{H}^{s, \beta^{(j+1)}}\left(M^{(j)}, F^{j}\right)$ with $\boldsymbol{M}=\left(M^{(j)}\right)_{0 \leq j \leq e}, M=M^{(0)}$, a tuple of vector bundles $\boldsymbol{F}=\left(F^{j}\right)_{0 \leq j \leq e}$ and tuples of weights $\beta=\left(\beta^{(j)}\right)_{0 \leq j \leq e}, \beta^{(j)}$ having $e-j+1$ components, for $1 \leq j \leq e, \beta^{(j)}=\emptyset$ for $j=e+1$ and $\mathcal{H}^{s, \beta^{(e+1)}}\left(M^{(e)}, F^{(e)}\right)=H^{s}\left(M^{(e)}, F^{(e)}\right)$ for $\operatorname{dim} M^{(e)}>0, \mathcal{H}^{s, \beta^{(e+1)}}\left(M^{(e)}, F^{(e)}\right)=\{0\}$ for $\operatorname{dim} M^{(e)}=0$,

(ii) spaces $\mathcal{L}^{\mu}\left(M, \boldsymbol{h} ; \boldsymbol{v} ; \Sigma \times \mathbb{R}^{l}\right)$ of parameter-dependent operator families of order $\mu \in \mathbb{R}$

$$
f(z, \zeta): \mathcal{H}^{s, \beta}(\boldsymbol{M}, \boldsymbol{F}) \rightarrow \mathcal{H}^{s-\mu, \beta-\mu}(\boldsymbol{M}, \tilde{\boldsymbol{F}}),
$$

with vector bundles $\boldsymbol{v}=(\boldsymbol{F}, \tilde{\boldsymbol{F}})$ and weight data $\boldsymbol{h}=(\beta, \beta-\mu)$, 
(iii) spaces of parameter-dependent principal symbols

$$
\operatorname{symb} \mathcal{L}^{\mu}\left(M, \boldsymbol{h} ; \boldsymbol{v} ; \Sigma \times \mathbb{R}^{l}\right),
$$

such that there is a linear surjective map

$$
\sigma: \mathcal{L}^{\mu}\left(M, \boldsymbol{h} ; \boldsymbol{v} ; \Sigma \times \mathbb{R}^{l}\right) \rightarrow \operatorname{symb} \mathcal{L}^{\mu}\left(M, \boldsymbol{h} ; \boldsymbol{v} ; \Sigma \times \mathbb{R}^{l}\right)
$$

with a right inverse op (an operator convention), where $\mathcal{A} \in \operatorname{ker} \sigma$ implies that (2.24) is compact for every $(z, \zeta) \in \Sigma \times \mathbb{R}^{l}$,

(iv) the spaces $\mathcal{L}^{\mu}\left(M, \boldsymbol{h} ; \boldsymbol{v} ; \Sigma \times \mathbb{R}^{l}\right)$ have natural locally convex topologies, and there is a notion of holomorphic functions of complex variables with values in these spaces,

(v) for $z=\left(z^{\prime}, z^{\prime \prime}\right) \in \Sigma^{\prime} \times \Sigma^{\prime \prime}, \zeta=\left(\zeta^{\prime}, \zeta^{\prime \prime}\right) \in \mathbb{R}^{l^{\prime}} \times \mathbb{R}^{l^{\prime \prime}}, \Sigma^{\prime} \subseteq \mathbb{R}^{l^{\prime}}, \Sigma^{\prime \prime} \subseteq \mathbb{R}^{l^{\prime \prime}}$, $l=l^{\prime}+l^{\prime \prime}$, and $f\left(z^{\prime}, z^{\prime \prime}, \zeta^{\prime}, \zeta^{\prime \prime}\right) \in \mathcal{L}^{\mu}\left(M, \boldsymbol{h} ; \boldsymbol{v} ; \Sigma^{\prime} \times \Sigma^{\prime \prime} \times \mathbb{R}^{l^{\prime}} \times \mathbb{R}^{l^{\prime \prime}}\right)$ we have

$$
\mathrm{op}_{z^{\prime}}(f)\left(z^{\prime \prime}, \zeta^{\prime \prime}\right) \in \mathcal{L}^{\mu}\left(\Sigma^{\prime} \times M, \boldsymbol{h} ; \boldsymbol{v} ; \Sigma^{\prime \prime} \times \mathbb{R}^{l^{\prime \prime}}\right)
$$

with op $z_{z^{\prime}}(f)\left(z^{\prime \prime}, \zeta^{\prime \prime}\right) u\left(z^{\prime}\right)=\iint e^{i\left(z^{\prime}-\tilde{z}^{\prime}\right) \zeta^{\prime}} f\left(z^{\prime}, z^{\prime \prime}, \zeta^{\prime}, \zeta^{\prime \prime}\right) u\left(\widetilde{z}^{\prime}\right) d \widetilde{z}^{\prime} d \zeta^{\prime} ;$ the involved bundles on $\Sigma^{\prime} \times M$ are corresponding liftings of bundles associated with $M$ and constitute $v$ over $\Sigma^{\prime} \times M$.

Remark 2.8 The notation in C4, where we use plain italics rather than boldface, indicates the fact that the orders are not to be understood in the DN-but in the usual sense, reached by order reductions by diagonal block matrices of the classes constructed in the same framework as those with $D N$-orders. A similar interpretation holds for the spaces when we do not employ boldface. To avoid confusion about wether we have $D N$-orders or usual orders in mind we want to emphasise that DNorders are preferred on infinite cones or Cartesian products between an open set in $\mathbb{R}$ and a compact singular manifold, while on a compact manifold with corners we mainly take the usual orders.

Remark 2.9 In C4 we referred to locally convex topologies in our operator spaces. The corresponding semi-norm systems become more and more complicated for growing singularity orders. However, to define holomorphic dependence of operators on parameters we can completely avoid this, if we treat complex variables in a similar manner as extra $C^{\infty}$ variables. This is possible in an inductive way by using holomorphy with values in an operator algebra on a space of lower singularity order and holomorphy with values in a space smoothing operators in the sense of a straightforward definition.

We call a manifold $M$ with singularities a manifold with edges if $M^{(e)}$ for $e=$ ord $M$ is a $C^{\infty}$ manifold of dimension $>0$. For $\operatorname{dim} M^{(e)}=0$, we talk about a manifold with corners, cf. Section 3 below.

Let us now give a definition of the spaces $\mathcal{H}_{\mathrm{loc}}^{s, \beta}(\boldsymbol{M}, \boldsymbol{F})$ and $\mathcal{H}_{\text {comp }}^{s, \beta}(\boldsymbol{M}, \boldsymbol{F})$ for non-compact $M$. Fix a locally finite open covering $\left(U_{\iota}\right)_{\iota \in I}$ of $M$ and $\left(\varphi_{\iota}\right)_{\iota \in I}$, $\varphi_{\iota} \in C^{\infty}\left(U_{\iota}\right)$, cf. Remark 1.2, a subordinate partition of unity (where for every $m \in M$ there are only finitely many $\iota \in I$ with $\left.\varphi_{l}(m) \neq 0\right)$. Consider the component $\mathcal{H}_{\mathrm{loc}}^{s, \beta}(M, F)\left(M=M^{(0)}, F=F^{(0)}\right)$; the constructions for $\left(M^{(j)}, F^{(j)}\right), j>0$, are analogous. The elements of $\mathcal{H}_{\mathrm{loc}}^{s, \beta}(M, F)$ are then defined to be sums $\sum_{\iota \in I} \varphi_{\iota} u_{\iota}$ for arbitrary $u_{\iota} \in \mathcal{H}_{\text {comp }}^{s, \beta}(M, F)$; so it remains to define $\mathcal{H}_{\text {comp }}^{s, \beta}(M, F)$. If $U_{\iota}$ is contained in $M \backslash M^{\prime}$ we set $\mathcal{H}_{\text {comp }}^{s, \beta}\left(U_{\iota}, F\right)=H_{\mathrm{loc}}^{s}\left(U_{\iota}, F\right)$; if $U_{\iota}$ is a corner neighbourhood, modelled by $N^{\Delta}$ for a compact $N$, ord $N<$ ord $M$, then $\mathcal{H}_{\text {comp }}^{s, \beta}\left(U_{\iota}, F\right)$ is 
the subspace of all elements of $\mathcal{K}^{s, \beta}\left(N^{\Delta}, F\right)$ with bounded support in $r \in \overline{\mathbb{R}}_{+}$; if $U_{L}$ is a wedge neighbourhood, modelled by $N^{\Delta} \times \Omega$, then $\mathcal{H}_{\text {comp }}^{s, \beta}\left(U_{\iota}, F\right)$ is the subspace of $\mathcal{W}_{\text {comp }}^{s}\left(\Omega, \mathcal{K}^{s, \beta}\left(N^{\Delta}, F\right)\right)$ with bounded support in $r \in \overline{\mathbb{R}}_{+}$.

For the invariance of these spaces under the transition maps for singular charts we choose an atlas for $M$, where the maps $\overline{\mathbb{R}}_{+} \times X_{1} \times \Omega_{1} \rightarrow \overline{\mathbb{R}}_{+} \times X_{2} \times \Omega_{2}$ are independent of the axial variable $r$ for $0 \leq r \leq \varepsilon$ for a small $\varepsilon>0$. We then get a correct global definition that is independent of the choice of $\left(U_{\iota}\right)_{\iota \in I},\left(\varphi_{l}\right)_{\iota \in I}$.

Remark 2.10 For a first inspection of our calculus it is reasonable to look at the spaces where all components of the weights equal s. This simplifies the formalism because the spaces have only one scale index s, cf. also Remark 4.4 below.

$\mathrm{C4}^{\prime}$ : On a manifold with singularities $M$ with ord $M \leq e$ (not necessarily compact) we impose analogous structures as in $\mathbf{C 4}$; the only difference is that the weighted Sobolev spaces are to be replaced by $\mathcal{H}_{\text {comp }}^{s, \beta}(\boldsymbol{M}, \boldsymbol{F})$ or $\mathcal{H}_{\mathrm{loc}}^{s, \beta}(\boldsymbol{M}, \boldsymbol{F})$ and $(2.24)$ by $f(z, \zeta): \mathcal{H}_{\mathrm{comp}}^{s, \beta}(\boldsymbol{M}, \boldsymbol{F}) \rightarrow \mathcal{H}_{\mathrm{loc}}^{s-\mu, \beta-\mu}(\boldsymbol{M}, \widetilde{\boldsymbol{F}})$, and the kernel of the symbol map does not necessarily consist of compact operators.

Let $B$ be a compact manifold with corners, i.e., there is a finite subset of corners $S \subset B$ such that ord $B \backslash S<$ ord $B$ (cf. also Remark 1.1); then $M:=B \backslash S$ is not compact, and the subscript comp in $\mathcal{H}_{\text {comp }}^{s, \beta}(\boldsymbol{M}, \boldsymbol{F})$ means that the corresponding elements vanish in a neighbourhood of $S$.

If $M$ is a manifold with singularities, ord $M \leq e, \mathcal{L}_{G}\left(M, \boldsymbol{h} ; \boldsymbol{v} ; \Sigma \times \mathbb{R}^{l}\right)$ for $\boldsymbol{v}=$ $(\boldsymbol{F}, \widetilde{\boldsymbol{F}})$ denotes the subspace of all $\mathcal{A}(z, \zeta) \in \mathcal{L}^{\mu}\left(M, \boldsymbol{h} ; \boldsymbol{v} ; \Sigma \times \mathbb{R}^{l}\right)$ such that there is an $\varepsilon=\varepsilon(\mathcal{A})>0$ with

$$
\begin{gathered}
\mathcal{A}(z, \zeta): \mathcal{H}_{\mathrm{comp}}^{s, \beta}(\boldsymbol{M}, \boldsymbol{F}) \rightarrow \mathcal{H}_{\mathrm{loc}}^{\infty, \beta-\mu+\varepsilon}(\boldsymbol{M}, \tilde{\boldsymbol{F}}), \\
\mathcal{A}^{*}(z, \zeta): \mathcal{H}_{\mathrm{comp}}^{s,-\beta+\mu}(\boldsymbol{M}, \widetilde{\boldsymbol{F}}) \rightarrow \mathcal{H}_{\mathrm{loc}}^{\infty,-\beta+\varepsilon}(\boldsymbol{M}, \boldsymbol{F}),
\end{gathered}
$$

such that $\mathcal{A}(z, \zeta)$ and $\mathcal{A}^{*}(z, \zeta)$ are $C^{\infty}$ functions in $z \in \Sigma$ and Schwartz functions in $\zeta \in \mathbb{R}^{l}$ with values in the respective spaces of continuous operators, for all $s \in \mathbb{R}$. The elements of $\mathcal{L}_{G}\left(M, \boldsymbol{h} ; \boldsymbol{v} ; \Sigma \times \mathbb{R}^{l}\right)$ are called Green operators.

Define $\mathcal{L}^{\mu}\left(M, \boldsymbol{h} ; \boldsymbol{v} ; \overline{\mathbb{R}}_{+} \times \Sigma \times \mathbb{R}^{l}\right)$ to be the space of all $\left.h(r, z, \zeta)\right|_{\overline{\mathbb{R}}_{+} \times \Sigma \times \mathbb{R}^{l}}$ for some $h(r, z, \zeta) \in \mathcal{L}^{\mu}\left(M, \boldsymbol{h} ; \boldsymbol{v} ; \mathbb{R} \times \Sigma \times \mathbb{R}^{l}\right)$.

Let $\mathcal{M}_{\mathcal{O}}^{\mu}\left(M, \boldsymbol{h} ; \boldsymbol{v} ; \overline{\mathbb{R}}_{+} \times \Sigma \times \Gamma_{\beta} \times \mathbb{R}^{l}\right), \beta \in \mathbb{R}$, be the space of all $h(r, z, w, \zeta) \in$ $\mathcal{L}^{\mu}\left(M, \boldsymbol{h} ; \boldsymbol{v} ; \overline{\mathbb{R}}_{+} \times \Sigma \times \Gamma_{\beta} \times \mathbb{R}^{l}\right)$ (with the (parameter) variables $(r, z) \in \overline{\mathbb{R}}_{+} \times \Sigma$ and covariables $\left.(w, \zeta) \in \Gamma_{\beta} \times \mathbb{R}^{l}\right)$ such that there is an $\varepsilon>0$ such that $h$ is holomorphic in $\{w: \beta-\varepsilon<\operatorname{Re} w<\beta+\varepsilon\}$ with values in $\mathcal{L}^{\mu}\left(M, \boldsymbol{h} ; \boldsymbol{v} ; \overline{\mathbb{R}}_{+} \times \Sigma \times \mathbb{R}^{l}\right)$ and

$$
\left.h\right|_{\overline{\mathbb{R}}_{+} \times \Sigma \times \Gamma_{\delta} \times \mathbb{R}^{l}} \in \mathcal{L}^{\mu}\left(M, \boldsymbol{h} ; \boldsymbol{v} ; \overline{\mathbb{R}}_{+} \times \Sigma \times \Gamma_{\delta} \times \mathbb{R}^{l}\right)
$$

for each $\beta-\varepsilon<\delta<\beta+\varepsilon$, uniformly in compact intervals.

Moreover, let $\mathcal{M}_{\mathcal{O}}^{\mu}\left(M, \boldsymbol{h} ; \boldsymbol{v} ; \overline{\mathbb{R}}_{+} \times \Sigma \times \mathbb{C} \times \mathbb{R}^{l}\right)$ defined to be the space of all $h \in \mathcal{A}\left(\mathbb{C}, \mathcal{L}^{\mu}\left(M, \boldsymbol{h} ; \boldsymbol{v} ; \overline{\mathbb{R}}_{+} \times \Sigma \times \mathbb{R}^{l}\right)\right)$ such that $(2.27)$ holds for all $\delta \in \mathbb{R}$, uniformly in compact intervals. The following type of Mellin operator convention has been introduced by the author in [55], cf. also [57]. Further useful constructions in this context may be found in the author's joint papers with Schrohe [48], [49], [52], Gil and Seiler [17], [16], as well as in Krainer [27].

C5: For every $p(r, z, \varrho, \zeta)=\widetilde{p}(r, z, r \varrho, \zeta)$ with $\tilde{p}(r, z, \widetilde{\varrho}, \zeta) \in \mathcal{L}^{\mu}\left(M, \boldsymbol{h} ; \boldsymbol{v} ; \overline{\mathbb{R}}_{+} \times \Sigma \times\right.$ $\left.\mathbb{R}_{\tilde{Q}, \zeta}^{1+l}\right)$ and arbitrary $\gamma \in \mathbb{R}$ there exists an $h(r, z, w, \zeta) \in \mathcal{M}_{\mathcal{O}}^{\mu}\left(M, \boldsymbol{h} ; \boldsymbol{v} ; \overline{\mathbb{R}}_{+} \times \Sigma \times \mathbb{C} \times\right.$ 
$\mathbb{R}^{l}$ ) such that

$$
\mathrm{op}_{r}(p)(z, \zeta)=\operatorname{op}_{M}^{\gamma}(h)(z, \zeta) \bmod \mathcal{L}^{-\infty}\left(\mathbb{R}_{+} \times M, \boldsymbol{h} ; \boldsymbol{v} ; \Sigma \times \mathbb{R}^{l}\right) .
$$

Here, $\mathrm{op}_{M}^{\gamma}(h)(z, \zeta) u(r)=M_{\gamma, w \rightarrow r}^{-1} M_{\gamma, r^{\prime} \rightarrow w}\left\{h\left(r^{\prime}, z, w, \zeta\right) u\left(r^{\prime}\right)\right\}$ with the weighted Mellin transform $\left(M_{\gamma} f\right)(w)=M\left(r^{-\gamma} f\right)(w+\gamma),(M g)(w)=\int_{0}^{\infty} r^{w-1} g(r) d r$.

According to the scenario of DN-orders in spaces of block-matrix operators, cf. (2.15), there is an evident analogue of the space $\mathcal{M}_{\mathcal{O}}^{\mu}\left(M, \boldsymbol{h}, \boldsymbol{v} ; \overline{\mathbb{R}}_{+} \times \Sigma \times \mathbb{C} \times \mathbb{R}^{l}\right)$, namely

$$
\begin{aligned}
\mathcal{M}_{\mathcal{O}}^{\mu}\left(M, \boldsymbol{h}, \boldsymbol{v} ; \overline{\mathbb{R}}_{+} \times \Sigma \times \mathbb{C} \times \mathbb{R}^{l}\right)=\left\{h=\left(h_{k j}\right)_{0 \leq k, j \leq e}:\right. \\
\left.h_{k j} \in \mathcal{M}_{\mathcal{O}}^{\mu_{k j}}\left(M, \boldsymbol{h}, \boldsymbol{v} ; \overline{\mathbb{R}}_{+} \times \Sigma \times \mathbb{C} \times \mathbb{R}^{l}\right) \text { for } j, k=0, \ldots, e\right\}
\end{aligned}
$$

with the same meaning of orders $\mu_{k j}$ as above. There is then a corresponding version of $\mathbf{C 5}$ that we can use in the sequel.

Let $(\eta, \zeta) \rightarrow[\eta, \zeta]$ be a strictly positive function in $C^{\infty}\left(\mathbb{R}^{q+l}\right)$ with $[\eta, \zeta]=|\eta, \zeta|$ for $|\eta, \zeta|>$ const for a constant $>0$.

W2: The space $\boldsymbol{R}^{\mu}\left(\Omega \times \mathbb{R}^{q}, \boldsymbol{g} ; \boldsymbol{v} ; \Sigma \times \mathbb{R}^{l}\right)$ for $\boldsymbol{g}=(\gamma, \gamma-\mu)$ and $\boldsymbol{v}=(\boldsymbol{E}, \widetilde{\boldsymbol{E}})$ is defined to be the set of all operator families

$$
\begin{aligned}
a(y, \eta ; z, \zeta)= & \boldsymbol{\omega}(r) r^{-\mu}\left\{\omega_{0}(r[\eta, \zeta]) \mathrm{op}_{M}^{\gamma_{e+1}-\frac{n}{2}}(h)(y, \eta ; z, \zeta) \omega_{1}(r[\eta, \zeta])\right. \\
& \left.+\left(1-\omega_{0}(r[\eta, \zeta])\right) P(y, \eta ; z, \zeta)\left(1-\omega_{2}(r[\eta, \zeta])\right)\right\} \widetilde{\boldsymbol{\omega}}(r) \\
& +g(y, \eta ; z, \zeta),
\end{aligned}
$$

with arbitrary cut-off functions $\boldsymbol{\omega}, \tilde{\boldsymbol{\omega}}, \omega_{k}, k=0,1,2$, satisfying $\omega_{0} \omega_{1}=\omega_{0},(1-$ $\left.\omega_{0}\right)\left(1-\omega_{2}\right)=1-\omega_{0}$, and

(i) $h(r, y, w, \eta ; z, \zeta)=\widetilde{h}(r, y, w, r \eta ; z, r \zeta)$ for some

$$
\tilde{h}(r, y, w, \eta ; z, \zeta) \in \mathcal{M}_{\mathcal{O}}^{\mu}\left(X, \boldsymbol{h} ; \boldsymbol{v} ; \overline{\mathbb{R}}_{+} \times \Omega \times \Sigma \times \Gamma_{\frac{n+1}{2}-\gamma_{e+1}} \times \mathbb{R}^{q+l}\right)
$$

with the variables $(r, y, z) \in \overline{\mathbb{R}}_{+} \times \Omega \times \Sigma$ and the covariables $(w, \eta, \zeta) \in$ $\Gamma_{\frac{n+1}{2}-\gamma_{e+1}} \times \mathbb{R}^{q} \times \mathbb{R}^{l}$; the weight data $\boldsymbol{h}$ are obtained from $\boldsymbol{g}$ by omitting $\gamma_{e+1}$ in the last component, cf. the notation in the beginning of Section 2,

(ii) $P(y, \eta ; z, \zeta)=\mathrm{op}_{r}(p)(y, \eta ; z, \zeta)$ for an element

$$
p(r, y, \varrho, \eta ; z, \zeta)=\tilde{p}(r, y, r \varrho, r \eta ; z, r \zeta)
$$

with $\tilde{p}(r, y, \tilde{\varrho}, \tilde{\eta} ; z, \widetilde{\zeta}) \in \mathcal{L}^{\mu}\left(X, \boldsymbol{h} ; \boldsymbol{v} ; \overline{\mathbb{R}}_{+} \times \Omega \times \Sigma \times \mathbb{R}_{\tilde{\varrho}, \tilde{\eta}, \tilde{\zeta}}^{1+q+l}\right)$ and

$$
\begin{aligned}
\operatorname{op}_{r}\left(\left.\tilde{p}\right|_{\tilde{\varrho}=r_{\varrho}}\right)(y, \tilde{\eta} ; z, \tilde{\zeta})=\operatorname{op}_{M}^{\gamma_{e+1}-\frac{n}{2}}(\tilde{h})(y, \tilde{\eta} ; z, \tilde{\zeta}) \\
\\
\bmod \mathcal{L}^{-\infty}\left(\mathbb{R}_{+} \times X, \boldsymbol{h} ; \boldsymbol{v} ; \Omega \times \Sigma \times \mathbb{R}_{\tilde{\eta}, \tilde{\zeta}}^{q+l}\right),
\end{aligned}
$$

(iii)

$$
\begin{aligned}
& g(y, \eta ; z, \zeta) \in \bigcap_{s \in \mathbb{R}} \boldsymbol{S}_{\mathrm{cl}}^{\mu}\left(\Omega \times \mathbb{R}^{q} ; \mathcal{K}^{s, \gamma}\left(\boldsymbol{X}^{\Delta}, \boldsymbol{E}\right), \mathcal{S}^{\gamma-\mu+\varepsilon}\left(\boldsymbol{X}^{\Delta}, \widetilde{\boldsymbol{E}}\right) ; \Sigma \times \mathbb{R}^{l}\right), \\
& g^{*}(y, \eta ; z, \zeta) \in \bigcap_{s \in \mathbb{R}} \boldsymbol{S}_{\mathrm{cl}}^{\mu}\left(\Omega \times \mathbb{R}^{q} ; \mathcal{K}^{s,(-\gamma+\mu)^{*}}\left(\boldsymbol{X}^{\Delta}, \widetilde{\boldsymbol{E}}\right), \boldsymbol{\mathcal { S }}^{(-\gamma)^{*}+\varepsilon}\left(\boldsymbol{X}^{\Delta}, \boldsymbol{E}\right) ; \Sigma \times \mathbb{R}^{l}\right)
\end{aligned}
$$

for some $\varepsilon>0$ (dependent on $g$ ), with $g^{*}$ being the pointwise formal adjoint. 
In our constructions it is possible to freeze coefficients, i.e., to form

$$
\begin{aligned}
\tilde{p}_{0}(y, \tilde{\varrho}, \tilde{\eta} ; z, \widetilde{\zeta}) & =\tilde{p}(0, y, \tilde{\varrho}, \tilde{\eta} ; z, \tilde{\zeta}) \\
& \in \mathcal{L}^{\mu}\left(X, \boldsymbol{h} ; \boldsymbol{v} ; \Omega \times \Sigma \times \mathbb{R}_{\tilde{\varrho}, \tilde{\eta}, \tilde{\zeta}}^{1+q+l}\right), \\
\tilde{h}_{0}(y, w, \tilde{\eta} ; z, \tilde{\zeta}) & =\widetilde{h}(0, y, w, \tilde{\eta} ; z, \tilde{\zeta}) \\
& \in \mathcal{M}_{\mathcal{O}}^{\mu}\left(X, \boldsymbol{h} ; \boldsymbol{v} ; \Omega \times \Sigma \times \Gamma_{\frac{n+1}{2}-\gamma_{e+1}} \times \mathbb{R}^{q+l}\right)
\end{aligned}
$$

Then we have

$$
\begin{aligned}
P_{0}(y, \eta ; z, \zeta):=\mathrm{op}_{r}\left(p_{0}\right)(y, \eta ; z, \eta)= & \operatorname{op}_{M}^{\gamma_{e+1}-\frac{n}{2}}\left(h_{0}\right)(y, \eta ; z, \eta) \\
& \bmod \mathcal{L}^{-\infty}\left(\mathbb{R}_{+} \times X, \boldsymbol{h} ; \boldsymbol{v} ; \Omega \times \Sigma \times \mathbb{R}_{\eta, \zeta}^{q+l}\right)
\end{aligned}
$$

for $p_{0}=\tilde{p}_{0}(y, r \varrho, r \eta ; z, r \zeta), h_{0}=\tilde{h}_{0}(y, w, r \eta ; z, r \zeta)$.

For the operator function $a(y, \eta ; z, \zeta)$ in $\mathbf{W} 2$ we now define a homogeneous principal symbol

$$
\begin{aligned}
\sigma_{\Lambda}(a)(y, \eta ; z, \zeta)= & r^{-\mu}\left\{\omega_{0}(r|\eta, \zeta|) \mathrm{op}_{M}^{\gamma_{e+1}-\frac{n}{2}}\left(h_{0}\right)(y, \eta ; z, \zeta) \omega_{1}(r|\eta, \zeta|)\right. \\
& \left.+\left(1-\omega_{0}(r|\eta, \zeta|)\right) P_{0}(y, \eta ; z, \zeta)\left(1-\omega_{2}(r|\eta, \zeta|)\right)\right\} \\
& +\sigma_{\Lambda}(g)(y, \eta ; z, \zeta),
\end{aligned}
$$

that is an $\mathcal{L}^{\mu}\left(X^{\Delta}, \boldsymbol{g} ; \boldsymbol{v}\right)$-valued function, parametrised by $(y, z, \eta, \zeta) \in T^{*}(\Omega \times \Sigma) \backslash 0$ (0 means $(\eta, \zeta)=0$ ). The summand $\sigma_{\Lambda}(g)$ is the homogeneous principal symbol in the sense of classical operator-valued symbols. The homogeneity of (2.28) means

$$
\sigma_{\Lambda}(a)(y, \lambda \eta ; z, \lambda \zeta)=\{\lambda\}^{\mu} \kappa_{\lambda} \sigma_{\Lambda}(a)(y, \eta ; z, \zeta) \kappa_{\lambda}^{-1}
$$

for all $(y, z, \eta, \zeta) \in T^{*}(\Omega \times \Sigma) \backslash 0, \lambda \in \mathbb{R}_{+}$, with $\left\{\kappa_{\lambda}\right\}_{\lambda \in \mathbb{R}_{+}}$being given by $\kappa_{\lambda}=$ $\bigoplus_{j=0}^{e} \kappa_{\lambda}^{(j)}$, cf. (2.3). Recall that $\{\lambda\}^{\mu}$ is an abbreviation for the factors $\lambda^{\mu_{k j}}$ that correspond to the homogeneities of the entries of the block-matrix.

More generally, for $a=\left(a_{i j}\right)_{i, j=1,2} \in \mathcal{R}^{\mu}\left(\Omega \times \mathbb{R}^{q}, \boldsymbol{g} ; \boldsymbol{w} ; \Sigma \times \mathbb{R}^{l}\right), \boldsymbol{w}=$ $\left(\boldsymbol{E}, N_{-} ; \widetilde{\boldsymbol{E}}, N_{+}\right)$, we introduce $\sigma_{\Lambda}(a)=\left(\sigma_{\Lambda}\left(a_{i j}\right)\right)_{i, j=1,2}$, where the entries $\sigma_{\Lambda}\left(a_{i j}\right)$ for $i+j>2$ are the homogeneous principal components of the corresponding classical operator-valued symbols. In other words, we have

$$
\sigma_{\Lambda}(a)(y, \eta ; z, \zeta): \underset{\mathbb{K}^{s, \gamma}\left(\boldsymbol{X}^{\Delta}, \boldsymbol{E}\right)}{\oplus} \rightarrow \begin{gathered}
\mathcal{K}^{s-\mu, \gamma-\mu}\left(\boldsymbol{X}^{\Delta}, \tilde{\boldsymbol{E}}\right) \\
\mathbb{C}^{N_{-}}
\end{gathered}
$$

and the homogeneity refers to the groups $\left\{\kappa_{\lambda}\right\}_{\lambda \in \mathbb{R}_{+}} \oplus \mathrm{id}_{\mathbb{C}^{N_{+}}}$and $\left\{\kappa_{\lambda}\right\}_{\lambda \in \mathbb{R}_{+}} \oplus \mathrm{id}_{\mathbb{C}^{N_{-}}}$, respectively, $(y, z, \eta, \zeta) \in T^{*}(\Omega \times \Sigma) \backslash 0$.

From W2 (ii) we have an element $\tilde{\widetilde{p}} \in \mathcal{L}^{\mu}\left(X, \boldsymbol{h} ; \boldsymbol{v} ; \mathbb{R} \times \Omega \times \Sigma \times \mathbb{R}^{1+q+l}\right), \boldsymbol{v}=$ $(\boldsymbol{E}, \tilde{\boldsymbol{E}})$, with

$$
\tilde{p}=\left.\tilde{\widetilde{p}}\right|_{\overline{\mathbb{R}}_{+} \times \Omega \times \Sigma \times \mathbb{R}^{1+q+i}},
$$

and $\sigma(\tilde{\widetilde{p}}) \in \operatorname{symb} \mathcal{L}^{\mu}\left(X, \boldsymbol{h} ; \boldsymbol{v} ; \mathbb{R} \times \Omega \times \Sigma \times \mathbb{R}^{1+q+l}\right)$ restricts to $\overline{\mathbb{R}}_{+} \times \Omega \times \Sigma$ in a canonical way. This gives us a space of principal symbols

$$
\operatorname{symb} \mathcal{L}^{\mu}\left(X, \boldsymbol{h} ; \boldsymbol{v} ; \overline{\mathbb{R}}_{+} \times \Omega \times \Sigma \times \mathbb{R}^{1+q+l}\right) .
$$

Set $\sigma_{\Phi}(\tilde{p})=\left.\sigma(\widetilde{\widetilde{p}})\right|_{\overline{\mathbb{R}}_{+} \times \Omega \times \Sigma}$. 
W3: The space of principal symbols symb $\mathcal{L}^{\mu}\left(X^{\Delta} \times \Omega, \boldsymbol{g} ; \boldsymbol{w} ; \Sigma \times \mathbb{R}^{l}\right), \boldsymbol{w}=$ $\left(\boldsymbol{E}, N_{-} ; \widetilde{\boldsymbol{E}}, N_{+}\right)$, is defined to be the set of all triples

$$
\sigma(\mathcal{A})=\left(\sigma_{\Psi}(\mathcal{A}), \sigma_{\Phi}(\mathcal{A}), \sigma_{\Lambda}(\mathcal{A})\right)
$$

with the "pseudo-differential" symbol $\sigma_{\Psi}(\mathcal{A}):=\sigma\left(\left.\mathcal{A}\right|_{\mathbb{R}_{+} \times X \times \Omega}\right)$ using $\left.\mathcal{A}\right|_{\mathbb{R}_{+} \times X \times \Omega} \in$ $\mathcal{L}^{\mu}\left(\mathbb{R}_{+} \times X \times \Omega, \boldsymbol{h} ; \boldsymbol{v} ; \Sigma \times \mathbb{R}^{l}\right)$, cf. C4 (iii), the Fuchs symbol $\sigma_{\Phi}(\mathcal{A}):=\sigma_{\Phi}(\tilde{\widetilde{p}})$, and the edge symbol $\sigma_{\Lambda}(\mathcal{A})=(2.30)$, where $\mathcal{A}$ is related to a by (2.22) and (2.28). Here, principal symbols are to be understood in the sense of DN-orders. Similarly, we define $\operatorname{symb} \mathcal{L}^{\mu}\left(X^{\Delta} \times \Omega, \boldsymbol{g} ; \boldsymbol{w} ; \Sigma \times \mathbb{R}^{l}\right)$ for the corresponding operator space with standard orders. Because of

$$
\mathcal{L}^{\mu}\left(X^{\Delta} \times \Omega, \boldsymbol{g} ; \boldsymbol{w} ; \Sigma \times \mathbb{R}^{l}\right) \cong \mathcal{L}^{\mu}\left(X^{\Delta} \times \Omega, \boldsymbol{g} ; \boldsymbol{w} ; \Sigma \times \mathbb{R}^{l}\right)
$$

via order reductions we can fix a bijection between symb $\mathcal{L}^{\mu}$ and $\operatorname{symb} \mathcal{L}^{\mu}$. It is then convenient to talk about the $D N$-version of $\left(\sigma_{\Psi}(\mathcal{A}), \sigma_{\Phi}(\mathcal{A}), \sigma_{\Lambda}(\mathcal{A})\right)$ for $\mathcal{A} \in \mathcal{L}^{\mu}$. If necessary we then write $\left(\mathrm{DN}-\sigma_{\Psi}(\mathcal{A}), \mathrm{DN}-\sigma_{\Phi}(\mathcal{A}), \mathrm{DN}-\sigma_{\Lambda}(\mathcal{A})\right.$ ) even though this depends on the chosen isomorphism (2.33).

Given any $\varphi(r, y) \in C_{0}^{\infty}\left(\overline{\mathbb{R}}_{+} \times \Omega\right)$ we form $\mathcal{M}_{\varphi}=\operatorname{diag}\left(\varphi,\left.\varphi\right|_{r=0}\right)$, the operator of multiplication by the corresponding diagonal block matrix. It can easily be proved that $\mathcal{A} \in \mathcal{L}^{\mu}\left(X^{\Delta} \times \Omega, \boldsymbol{g} ; \boldsymbol{v} ; \Sigma \times \mathbb{R}^{l}\right)$ implies $\mathcal{M}_{\varphi} \mathcal{A}, \mathcal{A} \mathcal{M}_{\varphi} \in \mathcal{L}^{\mu}\left(X^{\Delta} \times \Omega, \boldsymbol{g} ; \boldsymbol{v} ; \Sigma \times \mathbb{R}^{l}\right)$ (clearly, the multiplications by $\left.\varphi\right|_{r=0}$ refer to the dimensions $N_{ \pm}$, they disappear when $N_{-}$or $N_{+}$equal zero).

Theorem 2.11 $\mathcal{A} \in \mathcal{L}^{\mu}\left(X^{\Delta} \times \Omega, \boldsymbol{g} ; \boldsymbol{u} ; \Sigma \times \mathbb{R}^{l}\right)$ for $\boldsymbol{g}=(\gamma-\nu, \gamma-(\mu+\nu)), \boldsymbol{u}=$ $\left(\boldsymbol{F}, M ; \widetilde{\boldsymbol{E}}, N_{+}\right), \mathcal{B} \in \mathcal{L}^{\nu}\left(X^{\Delta} \times \Omega, \boldsymbol{c} ; \boldsymbol{w} ; \Sigma \times \mathbb{R}^{l}\right), \boldsymbol{c}=(\gamma, \gamma-\nu), \boldsymbol{w}=\left(\boldsymbol{E}, N_{-} ; \boldsymbol{F}, M\right)$, implies $\mathcal{A M}_{\varphi} \mathcal{B} \in \mathcal{L}^{\mu+\nu}\left(X^{\Delta} \times \Omega, \boldsymbol{g} \circ \boldsymbol{c} ; \boldsymbol{u} \circ \boldsymbol{w} ; \Sigma \times \mathbb{R}^{l}\right)$ where $\boldsymbol{g} \circ \boldsymbol{c}=(\gamma, \gamma-(\mu+\nu))$, $\boldsymbol{u} \circ \boldsymbol{w}=\left(\boldsymbol{E}, N_{-} ; \widetilde{\boldsymbol{E}}, N_{+}\right)$, and we have $\sigma\left(\mathcal{A} \mathcal{M}_{\varphi} \mathcal{B}\right)=\sigma\left(\mathcal{A M} \mathcal{M}_{\varphi}\right) \sigma(\mathcal{B})$ (with componentwise multiplication of the symbols).

The main idea of the proof is the fact that pseudo-differential operators with operator-valued symbols behave well under compositions, cf. [58], Theorem 14, and that the values of the symbols belong to parameter-dependent versions of algebras on singular manifolds of lower order that have been constructed before, where the composition result is known by the previous iteration step.

Definition 2.12 An operator $\mathcal{A}(z, \zeta) \in \mathcal{L}^{\mu}\left(X^{\Delta} \times \Omega, \boldsymbol{g} ; \boldsymbol{w} ; \Sigma \times \mathbb{R}^{l}\right)$ for $\boldsymbol{g}=(\gamma, \gamma-\mu)$ and $\boldsymbol{w}=\left(\boldsymbol{E}, N_{-} ; \widetilde{\boldsymbol{E}}, N_{+}\right)$is called (parameter-dependent) elliptic if

(i) $\mathcal{A}$ is $\sigma_{\Psi}$-elliptic, i.e., u. l.c. $\left.\mathcal{A}\right|_{\mathbb{R}_{+} \times X \times \Omega}$ is elliptic in the class $\mathcal{L}^{\mu}\left(\mathbb{R}_{+} \times X \times\right.$ $\left.\Omega, \boldsymbol{h} ; \boldsymbol{v} ; \Sigma \times \mathbb{R}^{l}\right), \boldsymbol{v}=(\boldsymbol{E}, \widetilde{\boldsymbol{E}})$,

(ii) $\mathcal{A}$ is $\sigma_{\Phi}$-elliptic, i.e., the operator function $\widetilde{p}(r, y, \widetilde{\varrho}, \eta ; z, \widetilde{\zeta})$ from W2 (ii) is of the form (2.31) for an elliptic element $\tilde{\widetilde{p}} \in \mathcal{L}^{\mu}\left(X, \boldsymbol{h} ; \boldsymbol{v} ; \mathbb{R} \times \Omega \times \Sigma \times \mathbb{R}^{1+q+l}\right)$,

(iii) $\mathcal{A}$ is $\sigma_{\Lambda}$-elliptic, i.e., the $D N-v e r s i o n$ of $\sigma_{\Lambda}(\mathcal{A})$ is a family of isomorphisms (2.30) for all $(y, z, \eta, \zeta) \in T^{*}(\Omega \times \Sigma) \backslash 0$ and some fixed $s \in \mathbb{R}$.

Remark 2.13 The bijectivity of (2.30) for an $s=s_{0} \in \mathbb{R}$ implies the bijectivity for all s. Condition (iii) is an analogue of the Shapiro-Lopatinskij condition in boundary value problems. We consider only this type of ellipticity here, although the existence of such conditions requires a topological property of u. l.c. $\mathcal{A}$, namely that the family of Fredholm operators

$$
\sigma_{\Lambda}(\text { u.l.c. } a)(y, \eta ; z, \zeta): \mathcal{K}^{s, \gamma}\left(\boldsymbol{X}^{\Delta}, \boldsymbol{E}\right) \rightarrow \mathcal{K}^{s-\mu, \gamma-\mu}\left(\boldsymbol{X}^{\Delta}, \widetilde{\boldsymbol{E}}\right)
$$


for $\left.(y, \eta ; z, \zeta) \in S^{*}(\Omega \times \Sigma)\right|_{\Delta}=: S^{*} \Delta$ (with $S^{*}(\Omega \times \Sigma)$ being the cosphere bundle induced by $T^{*}(\Omega \times \Sigma)$ and $\Delta \subset \subset \Omega \times \Sigma$ any compact subset) belongs to $\pi_{1}^{*} K(\Delta)$, where $\pi_{1}: S^{*} \Delta \rightarrow \Delta$ is the canonical projection and $K(\cdot)$ the $K$-group on the space in the brackets, cf. analogously Atiyah and Bott [1] or Boutet de Monvel [5]. There is a more general concept of ellipticity in terms of a generalisation of the APS-conditions; it is to be expected that our formalism from [64] can be adapted to the present situation.

Theorem 2.14 Let $\mathcal{A}(z, \zeta) \in \mathcal{L}^{\mu}\left(X^{\Delta} \times \Omega, \boldsymbol{g} ; \boldsymbol{w} ; \Sigma \times \mathbb{R}^{l}\right)$ be elliptic. Then there exists a parametrix $\mathcal{P}(z, \zeta) \in \mathcal{L}^{-\mu}\left(X^{\Delta} \times \Omega, g^{-1} ; \boldsymbol{w}^{-1} ; \Sigma \times \mathbb{R}^{l}\right)\left(\right.$ with $g^{-1}$ and $\boldsymbol{w}^{-1}$ being the data in opposite order $)$ of $\mathcal{A}(z, \zeta)$ in the sense that for arbitrary $\varphi(r, y), \psi(r, y) \in$ $C_{0}^{\infty}\left(\overline{\mathbb{R}}_{+} \times \Omega\right)$ with $\varphi \psi=\varphi$ we have

$$
\begin{aligned}
& \mathcal{P}(z, \zeta) \mathcal{M}_{\psi} \mathcal{A}(z, \zeta) \mathcal{M}_{\varphi}=\mathcal{M}_{\varphi} \bmod \mathcal{L}^{-\infty}\left(X^{\Delta} \times \Omega, \boldsymbol{g}_{l} ; \boldsymbol{w}_{l} ; \Sigma \times \mathbb{R}^{l}\right), \\
& \mathcal{A}(z, \zeta) \mathcal{M}_{\varphi} \mathcal{P}(z, \zeta) \mathcal{M}_{\psi}=\mathcal{M}_{\varphi} \bmod \mathcal{L}^{-\infty}\left(X^{\Delta} \times \Omega, \boldsymbol{g}_{r} ; \boldsymbol{w}_{r} ; \Sigma \times \mathbb{R}^{l}\right), \\
\text { for } \boldsymbol{g}_{l}= & (\gamma, \gamma), \boldsymbol{g}_{r}=(\gamma-\mu, \gamma-\mu) \text { and } \boldsymbol{w}_{l}=\left(\boldsymbol{E}, N_{-} ; \boldsymbol{E}, N_{-}\right), \boldsymbol{w}_{r}=\left(\widetilde{\boldsymbol{E}}, N_{+} ; \widetilde{\boldsymbol{E}}, N_{+}\right) .
\end{aligned}
$$

The proof of Theorem 2.14 is based on the fact that elliptic symbols have inverses within the symbol algebra symb $\mathcal{L}^{-\mu}\left(X^{\Delta} \times \Omega, g^{-1} ; \boldsymbol{w}^{-1} ; \Sigma \times \mathbb{R}^{l}\right)$. Then we can employ Theorem 2.11 and a formal Neumann series argument. The invertibility of symbols within the class can be proved in an analogous manner as for boundary value problems. First we construct an elliptic element $\mathcal{B} \in \mathcal{L}^{-\mu}\left(X^{\Delta} \times \Omega, \boldsymbol{g}^{-1} ; \boldsymbol{w}^{-1} ; \Sigma \times \mathbb{R}^{l}\right)$ with $\sigma_{\Psi}(\mathcal{B})=\sigma_{\Psi}(\mathcal{A})^{-1}$ and $\sigma_{\Phi}(\mathcal{B})=\sigma_{\Phi}(\mathcal{A})^{-1}$. This is a task of the parameterdependent cone theory that has been established before. Then we form (up to localisations) the composition $\mathcal{B} \mathcal{A}$ that equals $\mathcal{I}+\mathcal{C}$ where $\sigma_{\Psi}(\mathcal{C})=$ id and $\sigma_{\Phi}(\mathcal{C})=$ id. The inversion of $\sigma_{\Lambda}(\mathcal{I}+\mathcal{C})$ is similar to the inversion of boundary symbols of the form $1+g$ for a Green symbol $g$, cf. [63], Section 4.3.3.

The local constructions on wedges can be used to study analogous operator spaces globally on a manifold $M$ with edges, ord $M=e+1$, by using similar constructions as in the definition of global Sobolev spaces above.

By definition $Y:=M^{(e+1)}$ is a closed compact $C^{\infty}$ manifold of some dimension $q>0$, and we have $\operatorname{ord}(M \backslash Y)<$ ord $M$. Assume for simplicity that $Y$ has a neighbourhood $W$ in $M$ that is isomorphic to $X^{\Delta} \times Y$, where $X$ is a compact manifold with singularities, ord $X=e$. Consider a tuple $\boldsymbol{E}=\left(E^{j}\right)_{0 \leq j \leq e}$ of vector bundles on $W$ and their restrictions to bundles on $X^{(j)}$ (in the local representations), for brevity denoted by the same letters. The spaces $X^{(j) \Delta} \times Y$ are the local representatives of $M^{(j)}$ near $Y$, i.e., $M^{(j)} \cap W$ is isomorphic to $X^{(j) \Delta} \times Y, 0 \leq j \leq e$, and we assume that $E^{j}$ are restrictions of corresponding bundles on $M^{(j)}$ to $M^{(\bar{j})} \cap W \cong X^{(j) \Delta} \times Y$ that are also denoted by $E^{j}$. We then have the global spaces $\mathcal{H}^{s, \gamma^{(j+1)}}\left(M^{(j)}, E^{j}\right)$, and $\mathcal{H}^{s, \gamma}(\boldsymbol{M}, \boldsymbol{E}):=\bigoplus_{j=0}^{e} \mathcal{H}^{s, \gamma^{(j+1)}}\left(M^{(j)}, E^{j}\right)$ for $\boldsymbol{E}=\left(E^{j}\right)_{0 \leq j \leq e}$, cf. also C4 (i).

W4: Let $M$ be a manifold with edges $Y$; then $\mathcal{L}^{\mu}\left(M, \boldsymbol{g} ; \boldsymbol{w} ; \Sigma \times \mathbb{R}^{l}\right)$ for $\boldsymbol{g}=(\gamma, \gamma-\mu)$, $\boldsymbol{w}=\left(\boldsymbol{E}, J^{-}, \widetilde{\boldsymbol{E}}, J^{+}\right), \boldsymbol{E}=\left(E^{j}\right)_{0 \leq j \leq e}, \widetilde{\boldsymbol{E}}=\left(\widetilde{E}^{j}\right)_{0 \leq j \leq e}$ and bundles $J^{ \pm} \in \operatorname{Vect}(Y)$ of fibre dimensions $N_{ \pm}$, is the space of all parameter-dependent operators

$$
\mathcal{A}(z, \zeta): \underset{H_{\mathrm{comp}}^{s}\left(Y, J^{-}\right)}{\mathcal{H}_{\mathrm{comp}}^{s, \gamma}(\boldsymbol{M}, \boldsymbol{E})} \rightarrow \begin{gathered}
\mathcal{H}_{\mathrm{loc}}^{s-\mu, \gamma-\mu}(\boldsymbol{M}, \widetilde{\boldsymbol{E}}) \\
H_{\mathrm{loc}}^{s-\mu}\left(Y, J^{+}\right)
\end{gathered}
$$

such that 
(i) u. l.c. $\left.\mathcal{A}(z, \zeta)\right|_{M \backslash Y} \in \mathcal{L}^{\mu}\left(M \backslash Y, \boldsymbol{h} ; \boldsymbol{v} ; \Sigma \times \mathbb{R}^{l}\right)$,

(ii) $\chi_{*}\left(\left.\mathcal{A}(z, \zeta)\right|_{W}\right) \in \mathcal{L}^{\mu}\left(X^{\Delta} \times \Omega, \boldsymbol{g} ; \boldsymbol{w} ; \Sigma \times \mathbb{R}^{l}\right)$ for any wedge neighbourhood $W$ intersecting $Y$, where $\chi: W \rightarrow X^{\Delta} \times \Omega$ is a singular chart in the sense of Section 1, and $\boldsymbol{w}=\left(\boldsymbol{E}, N_{-} ; \widetilde{\boldsymbol{E}}, N_{+}\right)$,

(iii) $\mathcal{M}_{\varphi} \mathcal{A} \mathcal{M}_{\psi} \in \mathcal{L}_{G}\left(M, \boldsymbol{g} ; \boldsymbol{w} ; \Sigma \times \mathbb{R}^{l}\right)$ for every $\varphi, \psi \in C_{0}^{\infty}(M)$ with $\operatorname{supp} \varphi \cap$ $\operatorname{supp} \psi=\emptyset$, where $\mathcal{L}_{G}\left(M, \boldsymbol{g} ; \boldsymbol{w} ; \Sigma \times \mathbb{R}^{l}\right)$ is defined to be the space of all operator families $\mathcal{A}(z, \zeta)$ such that there is an $\varepsilon=\varepsilon(\mathcal{A})>0$ such that

$$
\mathcal{A}(z, \zeta): \underset{H_{\mathrm{comp}}^{s}\left(Y, J^{-}\right)}{\mathcal{H}_{\mathrm{comp}}^{s, \gamma}(\boldsymbol{M}, \boldsymbol{E})} \rightarrow \underset{H_{\mathrm{loc}}^{\infty}\left(Y, J^{+}\right)}{\mathcal{H}_{\mathrm{loc}}^{\infty, \gamma-\mu+\varepsilon}(\boldsymbol{M}, \widetilde{\boldsymbol{E}})}
$$

and

$$
\mathcal{A}^{*}(z, \zeta): \begin{gathered}
\mathcal{H}_{\mathrm{comp}}^{s,-\gamma+\mu}(\boldsymbol{M}, \widetilde{\boldsymbol{E}}) \\
H_{\mathrm{comp}}^{s}\left(Y, J^{+}\right)
\end{gathered} \rightarrow \begin{gathered}
\mathcal{H}_{\mathrm{loc}}^{\infty,-\gamma+\varepsilon}(\boldsymbol{M}, \boldsymbol{E}) \\
H_{\mathrm{loc}}^{\infty}\left(Y, J^{-}\right)
\end{gathered}
$$

are continuous and are $C^{\infty}$ functions in $z \in \Sigma$ and Schwartz functions in $\zeta \in \mathbb{R}^{l}$ with values in the corresponding operator spaces for all $s \in \mathbb{R}$,

(iv) $\operatorname{symb} \mathcal{L}^{\mu}\left(M, \boldsymbol{g} ; \boldsymbol{w} ; \Sigma \times \mathbb{R}^{l}\right)$ is the set of all triples

$$
\sigma(\mathcal{A})=\left(\sigma_{\Psi}(\mathcal{A}), \sigma_{\Phi}(\mathcal{A}), \sigma_{\Lambda}(\mathcal{A})\right)
$$

with $\sigma_{\Psi}(\mathcal{A})=\sigma_{\Psi}$ (u. l. c. $\left.\left.\mathcal{A}\right|_{M \backslash Y}\right) \in \operatorname{symb} \mathcal{L}^{\mu}\left(M \backslash Y, \boldsymbol{h} ; \boldsymbol{v} ; \Sigma \times \mathbb{R}^{l}\right)$, called the pseudo-differential symbol, further $\sigma_{\Phi}(\mathcal{A})$, the corresponding Fuchs symbol in a neighbourhood of $Y$, and

$$
\sigma_{\Lambda}(\mathcal{A})(y, \eta ; z, \zeta): \begin{gathered}
\mathcal{K}^{s, \gamma}\left(\boldsymbol{X}^{\Delta}, \boldsymbol{E}\right) \\
J_{y}^{-}
\end{gathered} \rightarrow \begin{gathered}
\mathcal{K}^{s-\mu, \gamma-\mu}\left(\boldsymbol{X}^{\Delta}, \widetilde{\boldsymbol{E}}\right) \\
J_{y}^{+}
\end{gathered}
$$

is the wedge symbol, parametrised by $T^{*}(Y \times \Sigma) \backslash 0$, invariantly defined by the system of edge symbols of the local representatives $\chi_{*}\left(\left.\mathcal{A}(z, \zeta)\right|_{W}\right)$ in the sense of (ii).

An operator $\mathcal{A}(z, \zeta) \in \mathcal{L}^{\mu}\left(M, \boldsymbol{g}, \boldsymbol{w} ; \Sigma \times \mathbb{R}^{l}\right)$ is called elliptic, if it is elliptic with respect to the components of $\sigma(\mathcal{A})$, where $\sigma_{\Psi}$-ellipticity is given from $\operatorname{ord}(M \backslash Y)<$ ord $M, \sigma_{\Phi}$-ellipticity is an evident generalisation of Definition 2.12 (ii), and $\sigma_{\Lambda^{-}}$ ellipticity is the bijectivity of (2.34) for all $(y, \eta ; z, \zeta) \in T^{*}(Y \times \Sigma) \backslash 0$.

If $\mathcal{A}(z, \zeta)$ is elliptic, there is a direct generalisation of Theorem 2.14. Let us explicitly formulate the corresponding result for the compact case under the abovementioned assumptions.

Theorem 2.15 Let $M$ be a compact manifold with edges $Y$ and $\mathcal{A}(z, \zeta) \in \mathcal{L}^{\mu}(M, \boldsymbol{g}$; $\left.\boldsymbol{w} ; \Sigma \times \mathbb{R}^{l}\right)$ be elliptic, $\boldsymbol{g}=(\gamma, \gamma-\mu), \boldsymbol{w}=\left(\boldsymbol{E}, J^{-} ; \widetilde{\boldsymbol{E}}, J^{+}\right)$. Then

$$
\mathcal{A}(z, \zeta): \underset{\oplus}{\mathcal{H}^{s, \gamma}(\boldsymbol{M}, \boldsymbol{E})} \rightarrow \underset{H^{s}\left(Y, J^{-}\right)}{\rightarrow} \rightarrow \begin{gathered}
\mathcal{H}^{s-\mu, \gamma-\mu}(\boldsymbol{M}, \tilde{\boldsymbol{E}}) \\
H^{s-\mu}\left(Y, J^{+}\right)
\end{gathered}
$$

is a Fredholm operator for all $s \in \mathbb{R}$ and $(z, \zeta) \in \Sigma \times \mathbb{R}^{l}$. For $l>0$ we have ind $\mathcal{A}(z, \zeta)=0$ for all $(z, \zeta) \in \Sigma \times \mathbb{R}^{l}$. There is a parametrix $\mathcal{P} \in \mathcal{L}^{-\mu}\left(M, g^{-1} ; \boldsymbol{w}^{-1}\right.$; $\left.\Sigma \times \mathbb{R}^{l}\right), \boldsymbol{g}^{-1}=(\gamma-\mu, \gamma), \boldsymbol{w}^{-1}=\left(\tilde{\boldsymbol{E}}, J^{+} ; \boldsymbol{E}, J^{-}\right)$, in the sense that

$$
\mathcal{P} \mathcal{A}-\mathcal{I} \in \mathcal{L}_{G}\left(M, \boldsymbol{g}_{l} ; \boldsymbol{w}_{l} ; \Sigma \times \mathbb{R}^{l}\right), \quad \mathcal{A P}-\mathcal{I} \in \mathcal{L}_{G}\left(M, \boldsymbol{g}_{r} ; \boldsymbol{w}_{r} ; \Sigma \times \mathbb{R}^{l}\right)
$$


for $\boldsymbol{g}_{l}=(\gamma, \gamma), \boldsymbol{w}_{l}=\left(\boldsymbol{E}, J^{-} ; \boldsymbol{E}, J^{-}\right)$and $\boldsymbol{g}_{r}=(\gamma-\mu, \gamma-\mu), \boldsymbol{w}_{r}=\left(\widetilde{\boldsymbol{E}}, J^{+} ; \widetilde{\boldsymbol{E}}, J^{+}\right)$.

The proof of the Fredholm property of $\mathcal{A}$ is a consequence of the existence of a parametrix $\mathcal{P}$ (cf. Theorem 2.14 for the local parametrix) and of the fact that for compact $M$ the elements of $\mathcal{L}_{G}$ are compact operators. This follows from the mapping properties of operators in $\mathcal{L}_{G}$ that transform the smoothness to $\infty$ and improve all weights by an $\varepsilon>0$. Then it suffices to observe that Sobolev spaces of smoothness $s^{\prime}$ and weights $\gamma^{\prime}$ are compactly embedded into spaces of smoothness $s<s^{\prime}$ and weights $\gamma<\gamma^{\prime}$.

Remark 2.16 The scenario of operator block matrices stems from the classical theory of (pseudo-differential) boundary value problems, cf. Vishik and Eskin [79], [80], Vishik and Grushin [81], Eskin [10], Sternin [76], Boutet de Monvel [5], and Rempel and Schulze [45]. Therefore, it is natural also to accept the presence of additional trace and potential operators as well as of Green operators as a central issue of the pseudo-differential analysis on spaces with polyhedral singularities (for our classes of degenerate operators, cf. Section 3 below). Many classical observations, e.g., reductions to the boundary (here, to lower-dimensional skeleta) or formulas of Agranovich-Dynin type for the index of elliptic operators, have analogues in our algebras, cf. Remark 4.2 below. Also "twisted" symbols in the sense of Definition 2.1 occur in classical boundary value problems, cf. Schulze [60] and [63], in particular, in symbol descriptions of Green's functions, see also [60] or the author's joint paper with Tarkhanov [69]. Analogous ideas seem to work for non-local elliptic boundary value problems in the spirit of APS boundary conditions, cf. Schulze [64], though for higher singularities this is not yet explicitly elaborated.

\section{Higher cone theories}

Our next objective is the pseudo-differential calculus on a cone $X^{\Delta}$, where $X$ is a compact manifold with singularities, ord $X=e$. In the iterative procedure we assume that the (parameter-dependent) calculus on $X$ (as well as on $X \times U$ for any open $U \subseteq \mathbb{R}^{q}$ ) is known and has the features formulated in C4 above. The role of the numbers $\mathbf{C} \boldsymbol{k}, \mathbf{W} \boldsymbol{j}, k, j \in \mathbb{N}$, in this section is that now $\mathbf{W} \boldsymbol{j}$ is used as given information, while $\mathbf{C} \boldsymbol{k}$ is created. The points $\mathbf{W} \boldsymbol{j}$ for $j>4$ basically belong to the preceding section, but their role becomes more transparent in connection with the cone structures.

Let us first introduce the relevant scales of Sobolev spaces. They are based on further properties of the spaces of operators on $X$.

Definition 3.1 The space $\mathcal{H}^{s, \gamma^{(j+1)}}\left(X^{(j) \Delta}, E^{j}\right)$ is defined by the following properties:

(i) $\mathcal{H}^{s, \gamma^{(j+1)}}\left(X^{(j) \Delta}, E^{j}\right)$ for $j>1$ is given by assumption because ord $X^{(j) \Delta} \leq e$ in this case,

(ii) $\mathcal{H}^{s, \gamma^{(1)}}\left(\mathbb{R}_{+} \times X^{(0)}, E^{0}\right)$ is given by assumption because of ord $\mathbb{R}_{+} \times X^{(0)}=e$,

(iii) let $v \in X^{(0)}$ be a corner point, i.e., $X^{(0)}$ is locally of the form $Y^{\Delta}$ for a $Y$ with ord $Y<e$, and $\nu:=\operatorname{dim}\left(Y \backslash Y^{\prime}\right)$; then

$$
\omega_{e} \omega_{e+1} \mathcal{H}^{s, \gamma^{(1)}}\left(X^{(0) \Delta}, E^{0}\right)=\omega_{e} \omega_{e+1} \mathcal{V}^{s, \gamma_{e+1}}\left(\mathbb{R}_{+}, \mathcal{K}^{s, \beta}\left(Y^{\Delta}, E^{0}\right)\right)
$$

where $\omega_{e}$ and $\omega_{e+1}$ are cut-off functions on the $r_{e+1}-$ and $r_{e}$-half-lines; moreover $\left(1-\omega_{e}\right) \omega_{e+1} \mathcal{H}^{s, \gamma^{(1)}}\left(X^{(0) \Delta}, E^{0}\right)$ is known by assumption, since $X^{(0) \Delta}$ is of order $e$ on the support of $\left(1-\omega_{e}\right) \omega_{e+1}$, 
(iv) let $x \in X^{(0)}$ be an edge point, i.e., $X^{(0)}$ is locally of the form $Y^{\Delta} \times \mathbb{R}^{q}$ for a manifold with singularities $Y$ with ord $Y<e$, then

$$
\varphi \omega_{e+1} \mathcal{H}^{s, \gamma^{(1)}}\left(X^{(0) \Delta}, E^{0}\right)=\varphi \omega_{e+1} \mathcal{V}^{s, \gamma_{e+1}}\left(\mathbb{R}_{+} \times \mathbb{R}^{q}, \mathcal{K}^{s, \beta}\left(Y^{\Delta}, E^{0}\right)\right),
$$

where $\varphi$ is any element in $C_{0}^{\infty}\left(X^{(0)}\right)$ of small support, contained in the corresponding wedge neighbourhood,

(v) if $\varphi \in C_{0}^{\infty}\left(X \backslash X^{\prime}\right)$, we set $\varphi \mathcal{H}^{s, \gamma^{(1)}}\left(X^{(0) \Delta}, E^{0}\right)=\varphi H_{\mathrm{loc}}^{s}\left(X \backslash X^{\prime}, E^{0}\right)$.

In this definition the points $r_{e+1}=0$ and $r_{e+1}=\infty$ are treated as conical singularities of $X^{(j) \Delta}$. We are, in fact, more interested in this interpretation for $r_{e+1}=0$, while we modify the spaces near $r_{e+1}=\infty$ in the sense that the "cylinder metric" of $\mathbb{R}_{+} \times X^{(j)}$ is replaced by a metric that treats $r_{e+1} \rightarrow \infty$ as an "exit to infinity", similarly to the standard metric in an Euclidean space near infinity. In the Euclidean space we have the usual Sobolev spaces, based on the Fourier transform; in general their behaviour is to be imitated. The precise definitions also require an iterative approach. An efficient way is to employ the operator functions $P(y, \eta ; z, \zeta)$ from W2, now in the simplest variant $P(\eta)$, i.e., without $(y, z, \zeta)$-dependence and $\boldsymbol{E}=\widetilde{\boldsymbol{E}}$. As operators on $\mathbb{R}_{+} \times X$ they are now of interest for $r_{e+1}>c$ for a constant $c>0$. For simplicity we set again $r:=r_{e+1}$. The underlying function $\tilde{p}(r, \tilde{\varrho}, \tilde{\eta}) \in$ $\mathcal{L}^{\mu}\left(X, \boldsymbol{h} ; \boldsymbol{E}, \boldsymbol{E} ; \overline{\mathbb{R}}_{+} \times \mathbb{R}_{\tilde{\varrho}, \tilde{\eta}}^{1+q}\right)$ with $P(\eta)=\mathrm{op}_{r}(p)(\eta), p(r, \varrho, \eta)=\tilde{p}(r, r \varrho, r \eta)$, will be chosen in a particular way: On the one hand we require an order reducing property for $r \geq c, \eta \neq 0$, on the other hand, for convenience, we extend the family from $[c, \infty) \times X$ to $\mathbb{R} \times X$ with an analogous behaviour for $-r \leq-c$ and order reducing property for all $r$. More precisely, by the iterative construction we have the following property:

W5: There exists an element

$$
\hat{p}(r, \tilde{\varrho}, \tilde{\eta})=\operatorname{diag}\left(\hat{p}_{j}(r, \tilde{\varrho}, \tilde{\eta})\right)_{j=0, \ldots, e} \in \mathcal{L}^{\mu}\left(X, \boldsymbol{h} ; \boldsymbol{E}, \boldsymbol{E} ; \mathbb{R} \times \mathbb{R}_{\tilde{\varrho}, \tilde{\eta}}^{1+q}\right)
$$

which is $r$-independent for $|r|>c$ for a given $c>0$ that induces isomorphisms

$$
\hat{p}_{j}(r, \tilde{\varrho}, \tilde{\eta}): \mathcal{H}^{s, \beta^{(j+1)}}\left(X^{(j)}, E^{j}\right) \rightarrow \mathcal{H}^{s-\mu, \beta^{(j+1)}-\mu}\left(X^{(j)}, E^{j}\right)
$$

for all $s \in \mathbb{R}$, and all $(r, \tilde{\varrho}, \tilde{\eta}) \in \mathbb{R} \times \mathbb{R}_{\tilde{\varrho}} \times \mathbb{R}_{\tilde{\eta}}, j=0, \ldots, e$, and we have $\hat{p}^{-1}(r, \tilde{\varrho}, \tilde{\eta}) \in$ $\mathcal{L}^{-\mu}\left(X, \boldsymbol{h}^{-1} ; \boldsymbol{E}, \boldsymbol{E} ; \mathbb{R} \times \mathbb{R}_{\tilde{\varrho}, \tilde{\eta}}^{1+q}\right)$.

Let us fix an $\eta_{1} \in \mathbb{R}^{q} \backslash 0$ and set $\hat{R}^{\mu}(r, \varrho)=r^{-\mu} \hat{p}\left(r, r \varrho, r \eta_{1}\right)$, cf. W2. The space $\mathcal{H}_{\sim}^{s, \beta}(\mathbb{R} \times \boldsymbol{X}, \boldsymbol{E}), s \in \mathbb{R}$, is defined to be the completion of $C_{0}^{\infty}\left(\mathbb{R}, \mathcal{H}^{s, \beta}(\boldsymbol{X}, \boldsymbol{E})\right)$ with respect to the norm

$$
\left\{\int_{-\infty}^{\infty}\left\|F_{r \rightarrow \varrho}\left\{\hat{R}^{s}(r, \varrho) u(r)\right\}\right\|_{\mathcal{H}_{\varrho}^{0, \beta-s}(\boldsymbol{X}, \boldsymbol{E})}^{2} d \varrho\right\}^{\frac{1}{2}} .
$$

Note that $\varphi \mathcal{H}_{\sim}^{s, \beta}(\mathbb{R} \times \boldsymbol{X}, \boldsymbol{E})=\varphi \mathcal{H}^{s, \beta}\left(\boldsymbol{X}^{\Delta}, \boldsymbol{E}\right)$ for every $\varphi \in C_{0}^{\infty}\left(\mathbb{R}_{+}\right)$.

Let us now describe the parameter-dependent norm that is used in formula (3.1). Let $X=X^{(0)}$ be a compact manifold with singularities, ord $X=e$, and set $E=E^{0}$. By assumption there is a finite covering $\left(V_{j}\right)_{j=1, \ldots, N}$ of $X$ such that there are charts $\chi_{j}: V_{j} \rightarrow Y_{j}^{\Delta} \times \Omega_{j}$ of order $<e$ and open sets $\Omega_{j} \subset \mathbb{R}^{q_{j}}$. The case that $Y_{j}^{\Delta}$ equals a point is also admitted; then the chart $V_{j} \rightarrow \Omega_{j}$ is regular. Assume for simplicity $\Omega_{j}=\mathbb{R}^{q_{j}}$. Choose a subordinate partition of unity $\left(\varphi_{j}\right)_{j=1, \ldots, N}$, 
$\varphi_{j} \in C_{0}^{\infty}\left(V_{j}\right)$. Set $v_{j}=\left(\chi_{j}^{-1}\right)^{*}\left(\varphi_{j} u\right)$ for $u \in \mathcal{H}^{s, \beta^{(1)}}(X, E), \beta^{(1)}=\left(\gamma_{1}, \ldots, \gamma_{e}\right)$ and $\|u\|_{\mathcal{H}^{s, \beta^{(1)}}(X, E)}=\left(\sum_{j=1}^{N}\left\|v_{j}\right\|_{j}^{2}\right)^{\frac{1}{2}}$, where

$\|v\|_{j}=\|v\|_{\mathcal{W}^{s}\left(\mathbb{R}^{\left.q_{j}, \mathcal{K}^{s, \beta(j)}\left(Y_{j}^{\Delta}, E\right)\right)}\right.}$ for $q_{j}>0, \quad\|v\|_{j}=\|v\|_{\left.\mathcal{K}^{s, \beta(j)}\left(Y_{j}^{\Delta}, E\right)\right)}$ for $q_{j}=0$.

Here, $E$ is the induced bundle on $Y_{j}^{\Delta}$ from the given bundle on $X$, and $\beta(j) \subset \beta^{(1)}$ is the tuple of weights that is associated with the system of axial variables in $Y_{j}^{\Delta}$. Note that for $Y_{j}^{\Delta}=\{$ point $\}$ we have $V_{j} \subset X \backslash X^{\prime}$ and $\|v\|_{j}=\|v\|_{H^{s}\left(\mathbb{R}^{n}\right)}, n=\operatorname{dim} X \backslash X^{\prime}$. In order to introduce the parameter-dependent norm on the space $\mathcal{H}^{s, \beta^{(1)}}(X, E)$ with parameter $\zeta \in \mathbb{R}^{l}$ we set

$\|v\|_{j, \zeta}=\|v\|_{\mathcal{W}_{\zeta}^{s}\left(\mathbb{R}^{\left.q_{j}, \mathcal{K}^{s, \beta(j)}\left(Y_{j}^{\Delta}, E\right)\right)}\right.}$ for $q_{j}>0, \quad\|v\|_{j, \zeta}=\|v\|_{\left.\mathcal{K}_{\zeta}^{s, \beta(j)}\left(Y_{j}^{\Delta}, E\right)\right)}$ for $q_{j}=0$

(where the latter parameter-dependent norm is defined in a similar manner as that for $\left.q_{j}>0\right)$ and $\|u\|_{\mathcal{H}_{\zeta}^{s, \beta(1)}(X, E)}=\left(\sum_{j=1}^{N}\left\|v_{j}\right\|_{j, \zeta}^{2}\right)^{\frac{1}{2}}$. Similarly, we define the parameter-dependent norms $\|\cdot\|_{\mathcal{H}_{\xi}^{s, \beta^{(j+1)}\left(X^{(j+1)}, E^{j}\right)}}, j=1, \ldots, e$, and then obtain $\|\cdot\|_{\mathcal{H}_{\zeta}^{s, \beta}(X, E)}$, cf. also the notation in C4 (i).

Definition 3.2 The weighted Sobolev space of smoothness $s \in \mathbb{R}$ and weights $\gamma=$ $\left(\gamma^{(j)}\right)_{1 \leq j \leq e+1}$ on the infinite cone is defined to be

$$
\mathcal{K}^{s, \gamma}\left(\boldsymbol{X}^{\Delta}, \boldsymbol{E}\right)=\omega \mathcal{H}^{s, \gamma}\left(\boldsymbol{X}^{\Delta}, \boldsymbol{E}\right)+\left.(1-\omega) \mathcal{H}_{\smile}^{s, \beta}(\mathbb{R} \times \boldsymbol{X}, \boldsymbol{E})\right|_{\mathbb{R}_{+} \times X}
$$

for any cut-off function $\omega(r)$. Moreover, if $B$ is a compact manifold with corner singularities (for simplicity we assume one corner singularity, modelled by $X^{\Delta}$ for a compact base $X)$, we set

$$
\mathcal{H}^{s, \gamma}(\boldsymbol{B}, \boldsymbol{E})=\omega \mathcal{H}^{s, \gamma}\left(\boldsymbol{X}^{\Delta}, \boldsymbol{E}\right)+(1-\omega) \mathcal{H}_{\mathrm{loc}}^{s, \beta}(\boldsymbol{B} \backslash \boldsymbol{S}, \boldsymbol{E})
$$

for any cut-off function $\omega$, supported in a small neighbourhood of $S$. Here, $\mathcal{H}_{\mathrm{loc}}^{s, \beta}(\boldsymbol{B} \backslash \boldsymbol{S}, \boldsymbol{E})$ is the space that is already defined on $B \backslash S$ as a manifold of lower order of singularity, cf. $\mathbf{C 4}^{\prime}$.

According to notation (2.16) we have also analogues of the space $\mathcal{H}^{s, \gamma}\left(\boldsymbol{X}^{\Delta}, \boldsymbol{E}\right)$ and $\mathcal{H}_{\triangle}^{s, \gamma}(\mathbb{R} \times \boldsymbol{X}, \boldsymbol{E})$ with $D N$-orders, denoted by

$$
\mathcal{H}^{s, \gamma}\left(\boldsymbol{X}^{\Delta}, \boldsymbol{E}\right) \quad \text { and } \quad \mathcal{H}_{\smile}^{s, \gamma}(\mathbb{R} \times \boldsymbol{X}, \boldsymbol{E}),
$$

respectively.

Let $\mathcal{L}_{\infty}^{\mu}\left(X, \boldsymbol{h} ; \boldsymbol{v} ; \overline{\mathbb{R}}_{+} \times \mathbb{R}_{\tilde{\varrho}}\right)$ for $\boldsymbol{v}=(\boldsymbol{E}, \widetilde{\boldsymbol{E}})$ (written in DN-orders with respect to the sequence $\left.X=X^{(0)} \supset X^{(1)} \supset \ldots\right)$ be the space of all $(r, \widetilde{\varrho})$-dependent operator families in $\mathcal{L}^{\mu}\left(X, \boldsymbol{h} ; \boldsymbol{v} ; \overline{\mathbb{R}}_{+} \times \mathbb{R}_{\tilde{\varrho}}\right)$ that have the form

$$
p_{\infty}(r, \tilde{\varrho})=\omega(r) p_{0}(r, \tilde{\varrho})+(1-\omega(r)) p_{1}\left(r, \tilde{\varrho}, r \eta_{1}\right)
$$

for a cut-off function $\omega(r)$ and elements $p_{0}(r, \widetilde{\varrho}) \in \mathcal{L}^{\mu}\left(X, \boldsymbol{h} ; \boldsymbol{v} ; \overline{\mathbb{R}}_{+} \times \mathbb{R}_{\tilde{\varrho}}\right), p_{1}(r, \tilde{\varrho}, \tilde{\eta})$ $\in \mathcal{L}^{\mu}\left(X, \boldsymbol{h} ; \boldsymbol{v} ; \overline{\mathbb{R}}_{+} \times \mathbb{R}_{\tilde{\varrho}, \tilde{\eta}}^{1+q}\right)$, for arbitrary $\eta_{1} \neq 0 ;$ in addition we assume that $p_{1}(r, \widetilde{\varrho}, \widetilde{\eta})$ is a classical symbol in $r$ of order zero for $r \rightarrow \infty$. The definition of the latter property is straightforward, so we do not go into details. In fact, in analogy to parameter-dependence of operators or amplitude functions on $r$ as $C^{\infty}$ functions we may impose a more specified behaviour in the sense of subspaces, e.g., symbol spaces in $r$ for $r \rightarrow \infty$. Let $\sigma_{r}^{(0)}\left(p_{1}\right)(\tilde{\varrho}, \tilde{\eta})$ be the homogeneous principal part in $r$ of order 0 and set $\sigma_{r}^{(0)}\left(p_{\infty}\right)(r, \varrho)=\sigma_{r}^{(0)}\left(p_{1}\right)\left(r \varrho, r \eta_{1}\right)$. 
C6: The space $\mathcal{C}^{\mu}\left(X^{\Delta}, \boldsymbol{g} ; \boldsymbol{v}\right), \boldsymbol{v}=(\boldsymbol{E}, \widetilde{\boldsymbol{E}})$, of all pseudo-differential operators of $(D N-)$ order $\mu$ on the infinite cone $X^{\Delta}$, of weight data $g=(\gamma, \gamma-\mu)$, is defined to be the set of all operators

$$
\mathcal{A}=r^{-\mu}\left\{\omega_{0}(r) \operatorname{op}_{M}^{\gamma_{e+1}-\frac{n}{2}}(h) \omega_{1}(r)+\left(1-\omega_{0}(r)\right) P\left(1-\omega_{2}(r)\right)\right\}+\mathcal{G},
$$

where $\omega_{0}, \omega_{1}, \omega_{2}$ are arbitrary cut-off functions with $\omega_{0} \omega_{1}=\omega_{0},\left(1-\omega_{0}\right)\left(1-\omega_{2}\right)=$ $1-\omega_{0}$, and

(i) $h(r, w) \in \mathcal{M}_{\mathcal{O}}^{\mu}\left(X, \boldsymbol{h} ; \boldsymbol{v} ; \overline{\mathbb{R}}_{+} \times \Gamma_{\frac{n+1}{2}-\gamma_{e+1}}\right), n=\operatorname{dim} X \backslash X^{\prime}$,

(ii) $P=\mathrm{op}_{r}(p)$ for an element $p(r, \varrho)=p_{\infty}(r, r \varrho)$ with $p_{\infty}(r, \widetilde{\varrho}) \in \mathcal{L}_{\infty}^{\mu}(X, \boldsymbol{h} ; \boldsymbol{v}$; $\left.\overline{\mathbb{R}}_{+} \times \mathbb{R}_{\hat{\varrho}}\right)$, with

$$
\mathrm{op}_{r}(p)=\mathrm{op}_{M}^{\gamma_{e+1}-\frac{n}{2}}(h) \bmod \mathcal{L}^{-\infty}\left(\mathbb{R}_{+} \times X, \boldsymbol{h} ; \boldsymbol{v}\right),
$$

(iii) $\mathcal{G}$ is a Green operator, defined by the mapping properties

$$
\begin{aligned}
& \mathcal{G}: \mathcal{K}^{s, \gamma}\left(\boldsymbol{X}^{\Delta}, \boldsymbol{E}\right) \rightarrow \mathcal{S}^{\gamma-\mu+\varepsilon}\left(\boldsymbol{X}^{\Delta}, \widetilde{\boldsymbol{E}}\right), \\
& \mathcal{G}^{*}: \mathcal{K}^{s,(-\gamma+\mu)^{*}}\left(\boldsymbol{X}^{\Delta}, \widetilde{\boldsymbol{E}}\right) \rightarrow \mathcal{S}^{(-\gamma)^{*}+\varepsilon}\left(\boldsymbol{X}^{\Delta}, \boldsymbol{E}\right)
\end{aligned}
$$

for some $\varepsilon>0$ (dependent on $\mathcal{G}$ ) with $\mathcal{G}^{*}$ being the formal adjoint.

The space $\mathcal{L}^{\mu}(B, \boldsymbol{g} ; \boldsymbol{v}), \boldsymbol{v}=(\boldsymbol{E}, \widetilde{\boldsymbol{E}})$, of all pseudo-differential operators of (standard) order $\mu$ on a compact manifold $B$ with corner singularities $S$ (say, one corner singularity, locally modelled by $\left.X^{\Delta}\right)$ is the space of all operators of the form (3.3) where $P \in \mathcal{L}^{\mu}(B \backslash S, \boldsymbol{h} ; \boldsymbol{v}), \omega_{0}, \omega_{1}, \omega_{2}$ are cut-off functions supported in a small neighbourhood of $S$, and $\mathcal{G}$ is a Green operator, defined by the mapping properties

$\mathcal{G}: \mathcal{H}^{s, \gamma}(\boldsymbol{B}, \boldsymbol{E}) \rightarrow \mathcal{H}^{\infty, \gamma-\mu+\varepsilon}(\boldsymbol{B}, \widetilde{\boldsymbol{E}}), \quad \mathcal{G}^{*}: \mathcal{H}^{s,-\gamma+\mu}(\boldsymbol{B}, \widetilde{\boldsymbol{E}}) \rightarrow \mathcal{H}^{\infty,-\gamma+\varepsilon}(\boldsymbol{B}, \boldsymbol{E})$

for all $s \in \mathbb{R}$ and some $\varepsilon>0$.

Denote the subspaces of Green operators in $\mathcal{C}^{\mu}\left(X^{\Delta}, \boldsymbol{g} ; \boldsymbol{v}\right)\left(\mathcal{L}^{\mu}(B, \boldsymbol{g} ; \boldsymbol{v})\right)$ by $\mathcal{C}_{G}\left(X^{\Delta}, \boldsymbol{g} ; \boldsymbol{v}\right)\left(\mathcal{L}_{G}(B, \boldsymbol{g} ; \boldsymbol{v})\right)$.

In the case of compact $B$ we can also define $\mathcal{L}^{\mu}(B, \boldsymbol{g} ; \boldsymbol{v})$ with arbitrary weight shift of the highest component of $\gamma$, but we omit this evident generalisation.

To $\mathcal{A} \in \mathcal{C}^{\mu}\left(X^{\Delta}, \boldsymbol{g} ; \boldsymbol{v}\right), \boldsymbol{v}=(\boldsymbol{E}, \widetilde{\boldsymbol{E}})$, we form the exit symbol

$$
\sigma_{e}(\mathcal{A})(r, \varrho)=r^{-\mu} \sigma_{r}^{(0)}\left(p_{\infty}\right)(r, \varrho)
$$

that describes the behaviour of $\mathcal{A}$ for $r \rightarrow \infty$; it belongs to $\mathcal{L}^{\mu}\left(X, \boldsymbol{h} ; \boldsymbol{v} ; \mathbb{R}_{+} \times \mathbb{R}_{\varrho}\right)$.

$\mathbf{C} \mathbf{6}$ is a special case of $\mathbf{W 2}$, so we can use the various pointwise properties of corresponding operator-valued symbols. In particular, every $\mathcal{A} \in \mathcal{C}^{\mu}\left(X^{\Delta}, \boldsymbol{g} ; \boldsymbol{v}\right)$ induces continuous operators

$$
\mathcal{A}: \mathcal{K}^{s, \gamma}\left(\boldsymbol{X}^{\Delta}, \boldsymbol{E}\right) \rightarrow \mathcal{K}^{s-\mu, \gamma-\mu}\left(\boldsymbol{X}^{\Delta}, \widetilde{\boldsymbol{E}}\right)
$$

for all $s \in \mathbb{R}$. Moreover, every $\mathcal{A} \in \mathcal{L}^{\mu}(B, \boldsymbol{g} ; \boldsymbol{E}, \widetilde{\boldsymbol{E}})$ induces continuous operators

$$
\mathcal{A}: \mathcal{H}^{s, \gamma}(\boldsymbol{B}, \boldsymbol{E}) \rightarrow \mathcal{H}^{s-\mu, \gamma-\mu}(\boldsymbol{B}, \widetilde{\boldsymbol{E}})
$$

for all $s \in \mathbb{R}$. They are compact for $\sigma(\mathcal{A})=0$.

C7: The space of principal symbols symb $\mathcal{C}^{\mu}\left(X^{\Delta}, \boldsymbol{g} ; \boldsymbol{v}\right), \boldsymbol{v}=(\boldsymbol{E}, \widetilde{\boldsymbol{E}})$, is defined to be the set of all tuples

$$
\sigma(\mathcal{A})=\left(\sigma_{\Psi}(\mathcal{A}), \sigma_{\Phi}(\mathcal{A}), \sigma_{c}(\mathcal{A}) ; \sigma_{e}(\mathcal{A})\right)
$$

with the "pseudo-differential" symbol $\sigma_{\Psi}(\mathcal{A})=\sigma\left(\left.\mathcal{A}\right|_{\mathbb{R}_{+} \times X}\right)$ from $\left.\mathcal{A}\right|_{\mathbb{R}_{+} \times X} \in$ $\mathcal{L}^{\mu}\left(\mathbb{R}_{+} \times X, \boldsymbol{h} ; \boldsymbol{v}\right)$, the Fuchs symbol $\sigma_{\Phi}(\mathcal{A}):=\sigma_{\Phi}(\tilde{p})$, where $\tilde{p}$ is defined by $(2.31)$ 
(here without $(y, \eta ; z, \zeta)$-variables), the conormal symbol $\sigma_{c}(\mathcal{A})(w):=h(0, w)$, regarded as an operator family

$$
\sigma_{c}(\mathcal{A})(w): \mathcal{H}^{s, \beta}(\boldsymbol{X}, \boldsymbol{E}) \rightarrow \mathcal{H}^{s-\mu, \beta-\mu}(\boldsymbol{X}, \tilde{\boldsymbol{E}}),
$$

parametrised by $w \in \Gamma_{\frac{n+1}{2}-\gamma_{e+1}}$, and the exit symbol $\sigma_{e}(\mathcal{A})$.

The space symb $\mathcal{L}^{\mu}(\stackrel{2}{2}, \boldsymbol{g} ; \boldsymbol{v})$ of principal symbols is defined to be the set of all triples

$$
\sigma(\mathcal{A})=\left(\sigma_{\Psi}(\mathcal{A}), \sigma_{\Phi}(\mathcal{A}), \sigma_{c}(\mathcal{A})\right)
$$

with the pseudo-differential symbol $\sigma_{\Psi}(\mathcal{A})=\sigma\left(\left.\mathcal{A}\right|_{B \backslash S}\right) \in \operatorname{symb} \mathcal{L}^{\mu}(B \backslash S, \boldsymbol{h} ; \boldsymbol{v})$, the Fuchs symbol $\sigma_{\Phi}(\mathcal{A})$, given in a neighbourhood of $S$ (in stretched coordinates), defined similarly to the case $X^{\Delta}$, and the conormal symbol $\sigma_{c}(\mathcal{A})$, that is (in $D N-$ orders) an operator family (3.6) for $w \in \Gamma_{\frac{n+1}{2}-\gamma_{e+1}}$, also defined as before.

Remark 3.3 The assumption in C6, C7 and in Theorem 3.6 below that $B$ is compact is not really essential. However in the compact case we can avoid comp/loc spaces. Clearly, there are natural generalisations of all constructions and results for manifolds $B$ with corners $S$ in the sense that $S$ is a finite subset and $\operatorname{ord}(B \backslash$ $S)<\operatorname{ord} B$ and the corresponding version of the second part of Theorem 3.6 below remains valid. An example is $B=X^{\Delta}$ with $S$ being the tip of the cone. The notation $\mathcal{C}^{\mu}\left(X^{\Delta}, \boldsymbol{g} ; \boldsymbol{v}\right)$ indicates a control of the exit properties of operators for $r \rightarrow \infty$.

Theorem 3.4 $\mathcal{A} \in \mathcal{C}^{\mu}\left(X^{\Delta}, \boldsymbol{g} ; \boldsymbol{F}, \widetilde{\boldsymbol{E}}\right)$ for $\boldsymbol{g}=(\gamma-\nu, \gamma-(\mu+\nu)), \mathcal{B} \in \mathcal{C}^{\nu}\left(X^{\Delta}, \boldsymbol{c} ; \boldsymbol{E}\right.$, $\boldsymbol{F})$ for $\boldsymbol{c}=(\gamma, \gamma-\nu)$ implies $\mathcal{A B} \in \mathcal{C}^{\mu+\nu}\left(X^{\Delta}, \boldsymbol{g} \circ \boldsymbol{c} ; \boldsymbol{E}, \widetilde{\boldsymbol{E}}\right)$, and we have $\sigma(\mathcal{A B})$ $\sigma(\mathcal{A}) \sigma(\mathcal{B})$, with componentwise multiplication, $\sigma_{c}(\mathcal{A B})=\left(T^{\nu} \sigma_{c}(\mathcal{A})\right) \sigma_{c}(\mathcal{B})$ for the conormal symbols; $\left(T^{\nu} f\right)(w):=f(w+\nu)$. An analogous composition result holds for operators on a compact space $B$ with corners.

The basic ideas of the proof are the same as those for Theorem 2.11 above.

Definition 3.5 An operator $\mathcal{A} \in \mathcal{C}^{\mu}\left(X^{\Delta}, \boldsymbol{g} ; \boldsymbol{v}\right), \boldsymbol{g}=(\gamma, \gamma-\mu), \boldsymbol{v}=(\boldsymbol{E}, \widetilde{\boldsymbol{E}})$, is called elliptic if

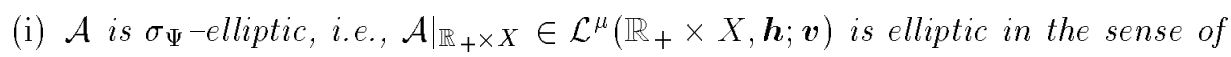
Definition 2.12,

(ii) $\mathcal{A}$ is $\sigma_{\Phi}$-elliptic, i.e., the operator function $\tilde{p}(r, \tilde{\varrho})$ from $\mathbf{C 6}$ (ii) is of the form $\tilde{p}=\left.\tilde{\widetilde{p}}\right|_{\mathbb{\mathbb { R }}_{+} \times \mathbb{R}}$ for an elliptic element $\widetilde{\widetilde{p}} \in \mathcal{L}^{\mu}(\mathbb{R} \times X, \widetilde{\boldsymbol{h}} ; \boldsymbol{v})$,

(iii) $\mathcal{A}$ is $\sigma_{\mathrm{c}}$-elliptic, i.e., the operators (3.6) are isomorphisms for some $s=s_{0} \in$ $\mathbb{R}$ and all $w \in \Gamma_{\frac{n+1}{2}}-\gamma_{e+1}$,

(iv) $\mathcal{A}$ is $\sigma_{e}$-elliptic, i.e., there is an $R>0$ such that

$$
\sigma_{e}(\mathcal{A})(r, \varrho): \mathcal{H}^{s, \beta}(\boldsymbol{X}, \boldsymbol{E}) \rightarrow \mathcal{H}^{s-\mu, \beta-\mu}(\boldsymbol{X}, \widetilde{\boldsymbol{E}})
$$

are isomorphisms for all $|r, \varrho| \geq R$ and an $s=s_{0} \in \mathbb{R}$, and there exists $a$ $q_{\infty}(r, \tilde{\varrho}) \in \mathcal{L}_{\infty}^{-\mu}\left(X, \boldsymbol{h}^{-1} ; \boldsymbol{v}^{-1} ; \overline{\mathbb{R}}_{+} \times \mathbb{R} \tilde{\varrho}\right), \boldsymbol{v}^{-1}=(\widetilde{\boldsymbol{E}}, \boldsymbol{E})$, such that

$$
\sigma_{e}(\mathcal{A})^{-1}(r, \varrho)=r^{\mu} \sigma_{r}^{(0)}\left(q_{\infty}\right)(r, \varrho)
$$

for all $|r, \varrho| \geq R$.

Analogously, an operator $\mathcal{A} \in \mathcal{L}^{\mu}(B, \boldsymbol{g} ; \boldsymbol{v})$ on a manifold $B$ with corners $S$ is called elliptic if $\mathcal{A}$ is $\left(\sigma_{\Psi}, \sigma_{\Phi}, \sigma_{c}\right)$-elliptic, i.e., (i) $\left.\mathcal{A}\right|_{B \backslash S}$ is elliptic in $\mathcal{L}^{\mu}(B \backslash S, \boldsymbol{h} ; \boldsymbol{v})$ and if $\mathcal{A}$ satisfies the analogues for standard orders of the conditions (ii), (iii) near the points of $S$ where $B$ is modelled by $X^{\Delta}$. 
Theorem 3.6 Let $\mathcal{A} \in \mathcal{C}^{\mu}\left(X^{\Delta}, \boldsymbol{g} ; \boldsymbol{v}\right), \boldsymbol{g}=(\gamma, \gamma-\mu), \boldsymbol{v}=(\boldsymbol{E}, \widetilde{\boldsymbol{E}})$, be elliptic. Then

$$
\mathcal{A}: \mathcal{K}^{s, \gamma}\left(\boldsymbol{X}^{\Delta}, \boldsymbol{E}\right) \rightarrow \mathcal{K}^{s-\mu, \gamma-\mu}\left(\boldsymbol{X}^{\Delta}, \tilde{\boldsymbol{E}}\right)
$$

is a Fredholm operator for every $s \in \mathbb{R}$, and $\mathcal{A}$ has a parametrix $\mathcal{P} \in \mathcal{C}^{-\mu}\left(X^{\Delta}, g^{-1}\right.$; $\left.\boldsymbol{v}^{-1}\right), g^{-1}=(\gamma-\mu, \gamma), \boldsymbol{v}^{-1}=(\widetilde{\boldsymbol{E}}, \boldsymbol{E})$, in the sense that

$$
\mathcal{P} \mathcal{A}-\mathcal{I} \in \mathcal{C}_{G}\left(X^{\Delta}, \boldsymbol{g}_{l} ; \boldsymbol{v}_{l}\right), \quad \mathcal{A P}-\mathcal{I} \in \mathcal{C}_{G}\left(X^{\Delta}, \boldsymbol{g}_{r} ; \boldsymbol{v}_{r}\right)
$$

where $\boldsymbol{g}_{l}=(\gamma, \gamma), \boldsymbol{v}_{l}=(\boldsymbol{E}, \boldsymbol{E}), \boldsymbol{g}_{r}=(\gamma-\mu, \gamma-\mu), \boldsymbol{v}_{r}=(\widetilde{\boldsymbol{E}}, \widetilde{\boldsymbol{E}})$. Analogously, if $\mathcal{A} \in \mathcal{L}^{\mu}(B, \boldsymbol{g} ; \boldsymbol{v})$ is elliptic and $B$ compact,

$$
\mathcal{A}: \mathcal{H}^{s, \gamma}(\boldsymbol{B}, \boldsymbol{E}) \rightarrow \mathcal{H}^{s-\mu, \gamma-\mu}(\boldsymbol{B}, \widetilde{\boldsymbol{E}})
$$

is a Fredholm operator for every $s \in \mathbb{R}$, and $\mathcal{A}$ has a parametrix $\mathcal{P} \in \mathcal{L}^{-\mu}\left(B, g^{-1}\right.$; $\left.v^{-1}\right)$ in the sense that

$$
\mathcal{P} \mathcal{A}-\mathcal{I} \in \mathcal{L}_{G}\left(B, \boldsymbol{g}_{l} ; \boldsymbol{v}_{l}\right), \quad \mathcal{A P}-\mathcal{I} \in \mathcal{L}_{G}\left(B, \boldsymbol{g}_{r} ; \boldsymbol{v}_{r}\right)
$$

In both cases we have $\sigma(\mathcal{P})=\sigma^{-1}(\mathcal{A})$.

The strategy of the proof is similar to that of Theorem 2.15. For the infinite cone $X^{\Delta}$ the ellipticity with respect to the exit symbol $\sigma_{c}$ implies a Schwartz space behaviour of the kernels of regularising operators near infinity.

\section{Concluding Remarks}

Summing up the results of Sections 2 and 3 we get on every (say compact) manifold $M$ with singularities

(i) an operator algebra $\mathcal{L}(M)=\bigcup_{\mu, \boldsymbol{g} ; \boldsymbol{w}} \mathcal{L}^{\mu}(M, \boldsymbol{g} ; \boldsymbol{w})$,

(ii) a principal symbol map $\sigma: \mathcal{L}(M) \rightarrow \operatorname{symb} \mathcal{L}(M)$ which determines ellipticity,

(iii) scales $\mathcal{H}^{s, \gamma}(\boldsymbol{M}, \boldsymbol{E})$ of spaces (that are direct sums of corresponding spaces of distributional sections in bundles $E^{j}$ on $M^{(j)}, 0 \leq j \leq$ ord $M$ ), and continuity of the operators in the spaces,

(iv) ellipticity implies the Fredholm property for all $s \in \mathbb{R}$, and ind $\mathcal{A}$ is independent of $s$; it only depends on $\sigma(\mathcal{A})$.

It is to be expected that the ellipticity of $\mathcal{A}$ is equivalent to the Fredholm property in Sobolev spaces, though this is still to be elaborated in detail. In the non-compact case the ellipticity implies the existence of a parametrix in the algebra.

From our inductive construction we easily obtain natural locally convex topologies in the spaces $\mathcal{L}^{\mu}(M, \boldsymbol{g} ; \boldsymbol{w})$ as well as in symb $\mathcal{L}^{\mu}(M, \boldsymbol{g} ; \boldsymbol{w})$. Homotopies of elliptic symbols $\left(\sigma_{t}\right)_{0 \leq t \leq 1}$ give rise to homotopies $\left(\mathcal{A}_{t}\right)_{0 \leq t \leq 1}$ of associated elliptic operators. Moreover, say for $\mu=0$, we can carry out stabilisations. Then the index of an elliptic operator $\mathcal{A}$ only depends on the stable homotopy class of $\sigma(\mathcal{A})$.

Remark 4.1 From the existence of parametrices of elliptic operators in the algebra and from the continuity properties we easily get elliptic regularity in weighted Sobolev spaces. The index is then independent of s. More precisely, the kernels are finite-dimensional subspaces of smoothness $\infty$ and of improved weight, and cokernels can also be represented by finite-dimensional subspaces of functions of analogous regularity; both are independent of $s$.

Remark 4.2 Let $\mathcal{A}_{1}, \mathcal{A}_{2} \in \mathcal{L}^{\mu}(M, \boldsymbol{g} ; \boldsymbol{w})$ be elliptic operators with the same upper left corners. Then we can compute an elliptic operator $\mathcal{B} \in \mathcal{L}^{0}\left(M^{\prime}, \boldsymbol{g}^{\prime} ; \boldsymbol{w}^{\prime}\right)$ 
for suitable weight data $g^{\prime}$ that refer to $M^{\prime}$ and tuples of bundles $\boldsymbol{w}^{\prime}$ on $\left(M^{\prime}, M^{\prime \prime}, \ldots, M^{(e)}\right), e=\operatorname{ord} M$, such that

$$
\text { ind } \mathcal{A}_{1}-\text { ind } \mathcal{A}_{2}=\text { ind } \mathcal{B} \text {. }
$$

This is an analogue of the Agranovich-Dynin formula. It follows in an analogous manner as the corresponding formula for boundary value problems, cf. Rempel and Schulze [44].

Remark 4.3 Our theory is formulated from the very beginning as a calculus with parameters (W2 gives a definition of parameter-dependent cone/corner theories; this can easily be modified to a corner theory with parameters for compact spaces with corners, cf. Schulze [59], Gil [15]). Both in edge and corner algebras (i.e., for a manifold $M$ with edges or corners in the sense of our notation) there are order reductions $R_{\boldsymbol{v}}^{s}: \mathcal{H}^{s, s}(\boldsymbol{M}, \boldsymbol{E}) \rightarrow \mathcal{H}^{0,0}(\boldsymbol{M}, \boldsymbol{E})$ for all $s \in \mathbb{R}, \boldsymbol{v}=(\boldsymbol{E}, \boldsymbol{E})$. For the edge case order reductions easily follow from the corresponding parameterdependent ellipticity, by taking the parameter large. For the corner case we can manage the bijectivity of operators directly by adding suitable smoothing (and not necessarily compact) Mellin and Green operators within the algebra.

Remark 4.4 The various technical elements of our operator algebras become easier if we restrict ourselves to operators of order zero and $\mathcal{H}^{0,0}$-spaces. Nevertheless, also in this situation, for the ellipticity we have to consider block matrices with additional trace and potential entries. To understand the stable homotopy classification of the ellipticity it suffices to look at the subalgebra of upper left corners, though this also contains a hierarchy of Green operators that are not easier than the full block matrices with trace and potential operators.

A generalisation of the various elements of the classical index theory to elliptic operators in the operator algebras is a program for the future. Our concept of ellipticity (or an analogue of the more general one from [68] or [64]) may be a starting point. A discussion of more details from the literature on index theory for singular configurations is not the intention of the present article. Our operator algebras with their symbol machinery are directly derived from the underlying singular configurations by a similar scheme as (pseudo-differential) boundary value problems on a $C^{\infty}$ manifold with boundary. These algebras deserve corresponding index theories for analogous reasons as classical boundary value problems, cf. Gelfand's program, sketched in the beginning.

Let us now explicitly describe the symbols of the operators u. l. c. $\left.\mathcal{A}\right|_{M \backslash M^{\prime}}$ for $\mathcal{A}(z, \zeta) \in \mathcal{L}^{\mu}\left(M, \boldsymbol{g} ; \boldsymbol{w} ; \Sigma \times \mathbb{R}^{l}\right)$ in stretched coordinates. For simplicity we omit the $z$-variables that are only involved with $C^{\infty}$ dependence.

In Section 1 for every $x_{0} \in M^{\prime}$ we produced a sequence of points $x_{j} \in X_{j}^{\prime}$, $0 \leq j \leq k-1$ and arrived at a stretched space $\left(\overline{\mathbb{R}}_{+}\right)^{k} \times X_{k} \times \Omega \times \ldots \times \Omega_{k}$. For the interior symbols it suffices to look at $\left(\overline{\mathbb{R}}_{+}\right)^{k} \times \times_{j=0}^{k} \Omega_{j}$ for open sets $\Omega_{j} \subseteq \mathbb{R}^{q_{j}}$, $j=0, \ldots, k$. Choose $l_{j} \in \mathbb{N}, j=1, \ldots, k$ and set $l=\sum_{j=1}^{k} l_{j}$.

Then the inductive procedure to generate operator algebras starts from the case $k=1$ and symbols of the form $p_{1}\left(r_{1}, x, \varrho_{1}, \xi\right)=r_{1}^{-\mu} \tilde{p}_{1}\left(r_{1}, x, r_{1} \varrho_{1}, \xi\right)$ that are Fuchs symbols with a weight factor $r_{1}^{-\mu}$, where $\widetilde{p}_{1}\left(r_{1}, x, \widetilde{\varrho}_{1}, \xi\right) \in S_{c l}^{\mu}\left(\overline{\mathbb{R}}_{+} \times \Omega_{0} \times \mathbb{R}_{\tilde{\varrho}_{1}, \xi}^{1+q_{0}}\right)$.

More generally, we may start from parameter-dependent edge-degenerate symbols of the variables $\left(r_{1}, x, y_{1}, \varrho_{1}, \xi, \eta_{1}, \zeta_{1}\right) \in \overline{\mathbb{R}}_{+} \times \Omega_{0} \times \Omega_{1} \times \mathbb{R}_{\varrho_{1}, \xi, \eta_{1}}^{1+q_{0}+q_{1}} \times \mathbb{R}_{\zeta_{1}}^{l_{1}}$ namely

$$
p_{1}\left(r_{1}, x, y_{1}, \varrho_{1}, \xi, \eta_{1}, \zeta_{1}\right)=r_{1}^{-\mu} \tilde{p}\left(r_{1}, x, y_{1}, r_{1} \varrho_{1}, \xi, r_{1} \eta_{1}, r_{1} \zeta_{1}\right)
$$


where $\tilde{p}\left(r_{1}, x, y_{1}, \widetilde{\varrho}_{1}, \xi, \tilde{\eta}_{1}, \widetilde{\zeta}_{1}\right) \in S_{\text {cl }}^{\mu}\left(\overline{\mathbb{R}}_{+} \times \Omega_{0} \times \Omega_{1} \times \mathbb{R}_{\tilde{\varrho}_{1}, \xi, \tilde{\eta}_{1}, \tilde{\zeta}_{1}}^{1+q_{0}+q_{1}+l_{1}}\right)$. This contains formally the first case for $q_{1}=l_{1}=0$. The next induction step leads to higherdegenerate symbols of the variables $(r, x, y, \varrho, \xi, \eta, \zeta) \in\left(\mathbb{R}_{+}\right)^{2} \times \Omega_{0} \times \Pi^{2} \times \mathbb{R}^{d_{2}}$, $r=\left(r_{1}, r_{2}\right), x \in \Omega_{0}, y=\left(y_{1}, y_{2}\right) \in \Pi^{2}:=\Omega_{1} \times \Omega_{2}, \varrho \in \mathbb{R}^{2}, \xi \in \mathbb{R}^{q_{0}}, \eta=\left(\eta_{1}, \eta_{2}\right) \in$ $\mathbb{R}^{q_{1}+q_{2}}, \zeta=\left(\zeta_{1}, \zeta_{2}\right) \in \mathbb{R}^{l_{1}+l_{2}}, d_{2}=2+\sum_{j=0}^{2} q_{j}+\sum_{m=1}^{2} l_{m}$, namely

$$
p_{2}(r, x, y, \varrho, \xi, \eta, \zeta)=\left(r_{1} r_{2}\right)^{-\mu} \tilde{p}_{2}\left(r, x, y, r_{1} \varrho_{1}, r_{1} r_{2} \varrho_{2}, \xi, r_{1} \eta_{1}, r_{1} r_{2} \eta_{2}, r_{1} \zeta_{1}, r_{1} r_{2} \zeta_{2}\right) \text {, }
$$

where $\widetilde{p}_{2}(r, x, y, \widetilde{\varrho}, \xi, \tilde{\eta}, \tilde{\zeta}) \in S_{\mathrm{cl}}^{\mu}\left(\left(\overline{\mathbb{R}}_{+}\right)^{2} \times \Omega_{0} \times \Pi^{2} \times \mathbb{R}^{d_{2}}\right)$.

For arbitrary $k \geq 1$ ( $k=e=$ ord $M$ in the above notation) we then get symbols

$$
\begin{gathered}
p_{k}(r, x, y, \varrho, \xi, \eta, \zeta)=\left(r_{1} r_{2} \ldots r_{k}\right)^{-\mu} \widetilde{p}_{k}\left(r, x, y, r_{1} \varrho_{1}, r_{1} r_{2} \varrho_{2}, \ldots, r_{1} r_{2} \ldots r_{k} \varrho_{k},\right. \\
\left.\xi, r_{1} \eta_{1}, r_{1} r_{2} \eta_{2}, \ldots, r_{1} r_{2} \ldots r_{k} \eta_{k}, r_{1} \zeta_{1}, r_{1} r_{2} \zeta_{2}, \ldots, r_{1} r_{2} \ldots r_{k} \zeta_{k}\right),
\end{gathered}
$$

where $\tilde{p}_{k}(r, x, y, \tilde{\varrho}, \xi, \tilde{\eta}, \tilde{\zeta}) \in S_{\text {cl }}^{\mu}\left(\left(\overline{\mathbb{R}}_{+}\right)^{k} \times \Omega_{0} \times \Pi^{k} \times \mathbb{R}^{d_{k}}\right)$ for $r \in\left(\mathbb{R}_{+}\right)^{k}, x \in \Omega_{0}$, $y \in \Pi^{k}:=\Omega_{1} \times \ldots \times \Omega_{k}, \varrho \in \mathbb{R}^{k}, \xi \in \mathbb{R}^{q_{0}}, \eta \in \mathbb{R}^{q_{1}+q_{2}+\ldots+q_{k}}, \zeta \in \mathbb{R}^{l_{1}+l_{2}+\ldots+l_{k}}$, $d_{k}=2+\sum_{j=0}^{k} q_{j}+\sum_{m=1}^{k} l_{m}$.

In other words our pseudo-differential calculus on $M$ is devoted to degenerate symbols of the form (4.1) for $k \leq$ ord $M$, cf. also formula (0.1); according to the choice of the corner or wedge neighbourhoods the dimensions $q_{j}, 0 \leq j \leq k$, satisfy the relation $\operatorname{dim}\left(M \backslash M^{\prime}\right)=k+\sum_{j=0}^{k} q_{j}$.

In a similar manner we can trace back the various operator-valued symbol components by successively inserting the representations of the symbols from earlier steps in the construction; this yields simpler symbols, dependent on corresponding stretched coordinates with degeneracies in the axial and edge covariables as well as in the $\zeta$-parameters.

Note that Maz'ja and Plameneskij [33] studied differential equations with symbols of the form (4.1), though without pseudo-differential techniques or additional edge trace and potential conditions. The weighted Sobolev spaces used in [33] are different from ours (for $s \neq \gamma$ and \{dimension of edges $>0$ ).

As emphasised in the beginning differential equations with degenerate symbols are a traditional field of the analysis, cf. Kondrat'ev and Oleynik [26] or Levendorskij and Paneah [32], and the bibliographies there. In recent years very much has been done for operators in geometric or topological problems, where the (differential) symbols are generated by vector fields with a specific behaviour near the singularities, cf. the work of Melrose [36] and his school.

The typical features of algebras of operators with prescribed symbols very much depend on the nature of degeneracies. For instance, we get a completely different scenario if instead of (4.1) we start from symbols $p(r, \varrho)=\tilde{p}(r, r \varrho)$, $r=\left(r_{1}, \ldots, r_{k}\right) \in\left(\overline{\mathbb{R}}_{+}\right)^{k}, r \varrho=\sum_{j=1}^{k} r_{j} \varrho_{j}$, where $\tilde{p}(r, \widetilde{\varrho}) \in S_{\text {cl }}^{\mu}\left(\left(\overline{\mathbb{R}}_{+}\right)^{k} \times \mathbb{R}_{\tilde{\varrho}}^{k}\right)$, cf. also Bolley and Camus [4].

Remark 4.5 Our degenerate symbols are also motivated by the form of LaplaceBeltrami operators to degenerate Riemannian metrics that model the warped polyhedral configurations.

In fact, consider a metric $d r_{1}^{2}+r_{1}^{2} g_{0}\left(r_{1}\right)$ for a family of Riemannian metrics $g_{0}\left(r_{1}\right)$ on $\Omega_{0}$, smoothly dependent on $r_{1} \in \overline{\mathbb{R}}_{+}$; then the associated Laplace-Beltrami operator has a symbol of the form (4.1) for $k=1$ and $\mu=2$ (without $(y, \eta, \zeta)$ and including the weight factor). Starting from a metric of the form $d r_{1}^{2}+r_{1}^{2} g_{0}\left(r_{1}, y_{1}\right)+$ 
$g_{1}\left(r_{1}, y_{1}\right)$ with families of Riemannian metrics $g_{j}\left(r_{1}, y_{1}\right)$ on $\Omega_{j}$ that are smooth in $\left(r_{1}, y_{1}\right) \in \overline{\mathbb{R}}_{+} \times \Omega_{1}$ for $j=0,1$, we get a Laplace-Beltrami operator with symbol (4.1) for $k=1$, including $\left(y_{1}, \eta_{1}\right)$-variables. For a metric of the form $d r_{2}^{2}+r_{2}^{2}\left\{d r_{1}^{2}+\right.$ $\left.r_{1}^{2} g_{0}\left(r_{1}, r_{2}, y_{1}, y_{2}\right)+g_{1}\left(r_{1}, r_{2}, y_{1}, y_{2}\right)\right\}+g_{2}\left(r_{1}, r_{2}, y_{1}, y_{2}\right)$ with families of Riemannian metrics $g_{j}(r, y)$ on $\Omega_{j}$ smoothly dependent on $(r, y) \in\left(\overline{\mathbb{R}}_{+}\right)^{2} \times \Omega_{1} \times \Omega_{2}$ for $j=0,1,2$, we get a symbol (4.1) for $k=2$.

This procedure of forming warped cones and wedges can be iterated and gives us examples of typical differential operators on a manifold $M$ with polyhedral singularities.

Differential operators of analogous structure also appear, if we start from a differential operator of order $\mu$ with $C^{\infty}$ coefficients in $\mathbb{R}^{N}$ for any $N$ and then successively introduce polar coordinates, first with respect to the origin in $\mathbb{R}^{N}$, then in the angular variables with respect to a point on $S^{N-1}$, and so on. Also in this case we get the typical weight factors. Clearly, differential operators with symbols (4.1) in general are much more singular than operators from such a polar coordinate construction. This shows that the class of degenerate symbols of the kind (4.1) contains the regular (i.e., non-degenerate) symbols with $C^{\infty}$ dependence on $r=\left(r_{1}, \ldots, r_{k}\right)$ up to $r=0$. In this sense the "singular analysis" with degenerate symbols is more general than the "regular" one. In particular, Boutet de Monvel's algebra [5] of boundary value problems with the transmission property (and also that without the transmission property [60]) is a substructure of the simplest version of the edge calculus, cf. [60]. Let us emphasise that for the formulations with degenerate symbols the weight factors in (4.1) are very natural also in connection with the orders of associated operator-valued symbols that are generated in the iteration of the edgification/conification process, cf. Definition 2.1 .

\section{REFERENCES}

[1] M.F. Atiyah and R. Bott, The index problem for manifolds with boundary, Coll. Differential Analysis, Tata Institute Bombay, Oxford University Press, 1964, pp. 175-186.

[2] M.F. Atiyah, V. Patodi, and I.M. Singer, Spectral asymmetry and Riemannian geometry I, II, III, Math. Proc. Cambridge Philos. Soc. 77,78,79 (1975, 1976, 1976), 43-69, 405-432, $315-330$.

[3] M.F. Atiyah and I.M. Singer, The index of elliptic operators I, II, III, Ann. of Math. (2) 87 (1968), 483-530, 531-545, 546-604.

[4] P. Bolley and J. Camus, Sur une classe d'opérateurs elliptiques et dégénérés à plusieurs variables, Bull. Soc. Math. France Mémoire 34 (1973), 55-140.

[5] L. Boutet de Monvel, Boundary problems for pseudo-differential operators, Acta Math. 126 (1971), 11-51.

[6] T. Carleman, Über das Neumann-Poincarésche Problem für ein Gebiet mit Ecken, Dissertation, Uppsala, 1916.

[7] Ch. Dorschfeldt, U. Grieme, and B.-W. Schulze, Pseudo-differential calculus in the Fourieredge approach on non-compact manifolds, Advances in Partial Differential Equations (Spectral Theory, Microlocal Analysis, Singular Manifolds), Akademie Verlag, Berlin, 1997, pp. $249-299$.

[8] Ju. V. Egorov and B.-W. Schulze, Pseudo-differential operators, singularities, applications, Birkhäuser Verlag, Basel, 1997.

[9] G.I. Eskin, Boundary value problems for elliptic pseudodifferential equations, Math. Monographs, vol. 52, Amer. Math. Soc., Providence, Rhode Island, 1980, Transl. of Nauka, Moskva, 1973.

[10] G.I. Eskin, Boundary-value problems for second-order elliptic equations in domains with corners, Proc. Sympos. Pure Math. 43 (1985), 105-131. 
[11] B.V. Fedosov and B.-W. Schulze, On the index of elliptic operators on a cone, Advances in Partial Differential Equations (Schrödinger Operators, Markov Semigroups, Wavelet Analysis, Operator Algebras), Akademie Verlag, Berlin, 1996, Preprint MPI 96-31, Max-Planck Institut, Bonn, 1996, pp. 348-372.

[12] B.V. Fedosov, B.-W. Schulze, and N.N. Tarkhanov, On the index of elliptic operators on a wedge, J. Funct. Anal. 157 (1998), 164-209.

[13] B.V. Fedosov, B.-W. Schulze, and N.N. Tarkhanov, On the index formula for singular surfaces, Pacific J. Math. 191, 1 (1999), 25-48.

[14] I.M. Gelfand, On elliptic equations, Uspekhi Mat. Nauk 15, 3 (1960), 121-132.

[15] J.B. Gil, Heat trace asymptotics for cone differential operators, Ph.D. thesis, University of Potsdam, 1998.

[16] J.B. Gil, B.-W Schulze, and J. Seiler, Holomorphic operator-valued symbols for edgedegenerate pseudo-differential operators, Differential Equations, Asymptotic Analysis, and Mathematical Physics, Math. Res., vol. 100, Akademie Verlag Berlin, 1997, pp. 113-137.

[17] J.B. Gil, B.-W Schulze, and J. Seiler, Cone pseudo-differential operators in the edge symbolic calculus, Osaka J. Math. 37 (2000), 219-258.

[18] P.B. Gilkey, Invariance theory, the heat equation, and the Atiyah-Singer index theorem, Studies in Advanced Mathematics, CRC Press, Boca Raton, Ann Arbor, London, Tokyo, 1995.

[19] I.C. Gohberg and E.I. Sigal, An operator generalization of the logarithmic residue theorem and the theorem of Rouché, Math. USSR Sbornik 13, 4 (1971), 603-625.

[20] M. Goresky and R. MacPherson, Stratified Morse theory, Springer Verlag, Berlin et al., 1988.

[21] H. Hertz, Über die Berührung fester elastischer Körper, J. Reine Angew. Math. 92 (1882), $156-171$.

[22] T. Hirschmann, Functional analysis in cone and edge Sobolev spaces, Ann. Global Anal. Geom. 8, 2 (1990), 167-192.

[23] D. Kapanadze, B.-W. Schulze, and I. Witt, Coordinate invariance of the cone algebra with asymptotics, Preprint, Institute for Mathematics, Potsdam, 2000, (in preparation).

[24] J.B. Keller, Wave propagation, Proc. ICM Zürich 1994, vol. 1, Birkhäuser Verlag, Basel, 1995, pp. 106-119.

[25] V.A. Kondrat'ev, Boundary value problems for elliptic equations in domains with conical points, Trudy Mosk. Mat. Obshch 16 (1967), 209-292.

[26] V.A. Kondrat'ev and O.A. Oleynik, Boundary problems for partial differential equations on non-smooth domains, Uspekhi Mat. Nauk 38, 2 (1983), 3-76.

[27] T. Krainer, Parabolic pseudo-differential operators and long-time asymptotics of solutions, Ph.D. thesis, University of Potsdam, 2000.

[28] G. Lamé, Leçons sur la théorie de la chaleur, Paris, 1861.

[29] R. Lauter, Holomorphic functional calculus in several variables and $\Psi^{*}$-algebras of totally characteristic operators on manifolds with boundary, Ph.D. thesis, University of Mainz, 1996.

[30] R. Lauter and J. Seiler, Pseudodifferential analysis on manifolds with boundary - a comparison of b-calculus and cone algebra, Advances in Partial Differential Equations (Approaches to Singular Analysis) (J. Gil, D. Grieser, and Lesch M., eds.), Oper. Theory Adv. Appl., Birkhäuser, Basel, 2000.

[31] M. Lesch, Operators of Fuchs type, conical singularities, and asymptotic methods, TeubnerTexte zur Mathematik, vol. 136, B.G. Teubner Verlagsgesellschaft, Stuttgart-Leipzig, 1997.

[32] S.Z. Levendorskij and B.P. Paneah, Degenerate elliptic equations and boundary problems, Partial Differential Equations VI (Yu.V. Egorov and M.A. Shubin, eds.), Encyclopedia of Mathematical Sciences, vol. 63, Springer-Verlag, Berlin et al., 1994, pp. 131-200.

[33] V.G. Maz'ja and B.A. Plameneskij, Elliptic boundary value problems on manifolds with singularities, Problems of Mathematical Analysis, vol. 6, Univ. Leningrad, 1977, pp. 85-142.

[34] R. Mazzeo, Elliptic theory of differential edge operators I, Comm. Partial Differential Equations 16 (1991), 1615-1664.

[35] H. Mellin, Über die fundamentale Wichtigkeit des Satzes von Cauchy für die Theorien der Gamma- und der hypergeometrischen Funktionen, Acta Soc. Fennicae 22, 1 (1886), 1-115.

[36] R.B. Melrose, Transformation of boundary problems, Acta Math. 147 (1981), 149-236.

[37] R.B. Melrose, The Atiyah-Patodi-Singer index theorem, Research Notes in Mathematics, A.K. Peters, Wellesley, 1993 
[38] R.B. Melrose and G.A. Mendoza, Elliptic operators of totally characteristic type, Preprint, MSRI, 1983.

[39] V. Nazaikinskij, B.-W. Schulze, and B. Ju. Sternin, On the homotopy classification of elliptic operators on manifolds with singularities, Preprint 99/21, Institute for Mathematics, Potsdam, 1999.

[40] V. Nazaikinskij, B.-W. Schulze, B. Ju. Sternin, and V. Shatalov, Spectral boundary value problems and elliptic equations on singular manifolds, Differentsial'nye Uravneniya 34, 5 (1998), 695-708, (Russian).

[41] Nistor P., A. Weinstein, and P. Xu, Pseudodifferential operators on differential groupoids, Pacific J. Math. 189, 1 (1999), 117-152.

[42] B.A. Plamenevskij, Algebras of pseudo-differential operators, Nauka, Moscow, 1986.

[43] V.S. Rabinovich, B.-W. Schulze, and N. Tarkhanov, Boundary value problems in domains with corners, Preprint 99/19, Institute for Mathematics, Potsdam, 1999.

[44] S. Rempel and B.-W. Schulze, Index theory of elliptic boundary problems, Akademie-Verlag, Berlin, 1982.

[45] S. Rempel and B.-W. Schulze, Parametrices and boundary symbolic calculus for elliptic boundary problems without transmission property, Math. Nachr. 105 (1982), 45-149.

[46] G. Rozenblum, Index formulae for pseudodifferential operators with discontinuous symbols, Ann. Global Anal. Geom. 15 (1997), 71-100.

[47] A. Savin, B.-W. Schulze, and B. Sternin, Elliptic operators in subspaces and the eta invariant, Preprint 99/14, Institute for Mathematics, Potsdam, 1999.

[48] E. Schrohe and B.-W. Schulze, Boundary value problems in Boutet de Monvel's calculus for manifolds with conical singularities I, Advances in Partial Differential Equations (PseudoDifferential Calculus and Mathematical Physics), Akademie Verlag, Berlin, 1994, pp. 97-209.

[49] E. Schrohe and B.-W. Schulze, Boundary value problems in Boutet de Monvel's calculus for manifolds with conical singularities II, Advances in Partial Differential Equations (Boundary Value Problems, Schrödinger Operators, Deformation Quantization), Akademie Verlag, Berlin, 1995, pp. 70-205.

[50] E. Schrohe and B.-W. Schulze, Mellin operators in a pseudodifferential calculus for boundary value problems on manifolds with edges. IWOTA 95 Proceedings, "Differential and Integral Operators" (C. Mennicken and C. Tretter, eds.), Operator Theory: Advances and Applications, vol. 102, Birkhäuser Verlag, Basel, 1997, pp. 257-285.

[51] E. Schrohe and B.-W. Schulze, A symbol algebra for pseudodifferential boundary value problems on manifolds with edges, Differential Equations, Asymptotic Analysis, and Mathematical Physics, Math. Research, vol. 100, Akademie Verlag, Berlin, 1997, pp. 292-324.

[52] E. Schrohe and B.-W. Schulze, Mellin and Green symbols for boundary value problems on manifolds with edges, Integral Equations Operator Theory 34 (1999), 339-363.

[53] E. Schrohe and J. Seiler, Ellipticity and invertibility in the cone algebra on $L_{p}-$ Sobolev spaces, Preprint 99/28, Institute for Mathematics, Potsdam, 1999.

[54] B.-W. Schulze, Ellipticity and continuous conormal asymptotics on manifolds with conical singularities, Math. Nachr. 136 (1988), 7-57.

[55] B.-W. Schulze, Corner Mellin operators and reduction of orders with parameters, Ann. Scuola Norm. Sup. Pisa Cl. Sci. 16, 1 (1989), 1-81.

[56] B.-W. Schulze, Pseudo-differential operators on manifolds with edges, Symposium "Partial Differential Equations", Holzhau 1988, Teubner-Texte zur Mathematik, vol. 112, Teubner, Leipzig, 1989, pp. 259-287.

[57] B.-W. Schulze, Mellin representations of pseudo-differential operators on manifolds with corners, Ann. Glob. Anal. Geom. 8, 3 (1990), 261-297.

[58] B.-W. Schulze, Pseudo-differential operators on manifolds with singularities, North-Holland, Amsterdam, 1991.

[59] B.-W. Schulze, The Mellin pseudo-differential calculus on manifolds with corners, Symposium: "Analysis in Domains and on Manifolds with Singularities", Breitenbrunn 1990, Teubner-Texte zur Mathematik, vol. 131, Teubner, Leipzig, 1992, pp. 208-289.

[60] B.-W. Schulze, Pseudo-differential boundary value problems, conical singularities, and asymptotics, Akademie Verlag, Berlin, 1994.

[61] B.-W. Schulze, The variable discrete asymptotics in pseudo-differential boundary value problems I, Advances in Partial Differential Equations (Pseudo-Differential Calculus and Mathematical Physics), Akademie Verlag, Berlin, 1994, pp. 9-96. 
[62] B.-W. Schulze, The variable discrete asymptotics in pseudo-differential boundary value problems II, Advances in Partial Differential Equations (Boundary Value Problems, Schrödinger Operators, Deformation Quantization), Akademie Verlag, Berlin, 1995, pp. 9-69.

[63] B.-W. Schulze, Boundary value problems and singular pseudo-differential operators, J. Wiley, Chichester, 1998.

[64] B.-W. Schulze, An algebra of boundary value problems not requiring Shapiro-Lopatinskij conditions, Preprint 99/24, Institute for Mathematics, Potsdam, 1999, J. Funct. Anal. (to appear).

[65] B.-W. Schulze, Iterated asymptotics on corner manifolds. Proceedings PDE Conference Wuhan, April 5-9, 1999, World Scientific Publishing Co. of Singapore, 2000.

[66] B.-W. Schulze, Operators on corner manifolds, J. Wiley, (in preparation).

[67] B.-W. Schulze, B. Sternin, and V. Shatalov, Differential equations on singular manifolds. Semiclassical theory and operator algebras, Wiley-VCH, Weinheim, 1999.

[68] B.-W. Schulze, B.Ju. Sternin, and V.E. Shatalov, On general boundary value problems for elliptic equations, Sbornik:Mathematics 189, 10 (1998), 1573-1586.

[69] B.-W. Schulze and N.N. Tarkhanov, Green pseudodifferential operators on a manifold with edges, Comm. Part. Diff. Equ. 23, 1-2 (1998), 171-200.

[70] B.-W. Schulze and N.N. Tarkhanov, Ellipticity and parametrices on manifolds with cuspidal edges, Geometric Aspects in Partial Differential Equations (B. Booss-Bavnbeck and K. Wojciechowski, eds.), Contemporary Mathematics, vol. 242, Amer. Math. Society, Providence, R.I., 1999, pp. 217-256.

[71] B.-W. Schulze and N.N. Tarkhanov, Euler solutions of pseudodifferential equations, Integral Equations Operator Theory 33 (1999), 98-123.

[72] B.-W. Schulze and G. Wildenhain, Methoden der Potentialtheorie für Elliptische Differentialgleichungen beliebiger Ordnung, Akademie-Verlag, Berlin; Birkhäuser Verlag, Basel, 1977.

[73] J. Seiler, Pseudodifferential calculus on manifolds with non-compact edges, Ph.D. thesis, University of Potsdam, 1998.

[74] S.L. Sobolev, Einige Anwendungen der Funktionalanalysis auf Gleichungen der mathematischen Physik, Akademie-Verlag, Berlin, 1964.

[75] A. Sommerfeld, Partielle Differentialgleichungen der Physik (5. Auflage), Akademische Verlagsgesellschaft Geest \& Portig K.-G., Leipzig, 1962.

[76] B.Ju. Sternin, Topological aspects of a problem of S. L. Sobolev, Mosk. inst. elektr. mashinostroenija, Moskva, 1971.

[77] N. Teleman, Global analysis on PL-manifolds, Trans. A.M.S. 256 (1979), 49-88.

[78] A. Unterberger and H. Upmeier, Pseudodifferential analysis on symmetric cones, Studies in Advanced Mathematics, CRC Press Boca Raton, New York, London, Tokyo, 1996.

[79] M.I. Vishik and G.I. Eskin, Convolution equations in a bounded region, Uspekhi Mat. Nauk 20, 3 (1965), 89-152

[80] M.I. Vishik and G.I. Eskin, Convolution equations in bounded domains in spaces with weighted norms, Mat. Sb. 69, 1 (1966), 65-110.

[81] M.I. Vishik and V.V. Grushin, On a class of degenerate elliptic equations of higher orders, Mat. Sb. 79, 1 (1969), 3-36.

[82] N. Wiener, The Dirichlet problem, J. Math. Physics 3 (1924), 127-146.

[83] I. Witt, Explicit algebras with the Leibniz-Mellin translation product, Preprint 99/2, Institute for Mathematics, Potsdam, 1999.

[84] I. Witt, On the factorization of meromorphic Mellin symbols, Preprint 99/5, Institute for Mathematics, Potsdam, 1999.

[85] S. Zaremba, Sur un problème mixte relatif à l'equation de Laplace, Bull. de l'Academie des Sciences de Cracovie, Class. math. et nat., Sér. A (1910), 313-344.

Institut für Mathematik, Universität Potsdam, PF 6015 53, 14469 Potsdam

E-mail address: schulze@math.uni-potsdam.de 